\title{
Galaxies as simple dynamical systems: observational data disfavor dark matter and stochastic star formation
}

\author{
Pavel Kroupa
}

\begin{abstract}
According to modern theory galactic evolution is driven by the dynamics of dark matter and stochastic star formation, but galaxies are observed to be simple systems. The existence of dark matter particles is a key hypothesis in present-day cosmology and galactic dynamics. Given the large body of high-quality work within the standard model of cosmology (SMoC), the validity of this hypothesis is challenged significantly by two independent arguments: (1) The dual dwarf galaxy theorem must be true in any realistic cosmological model. But the now available data appear to falsify it when the dark-matter-based model is compared with the observational data. A consistency test for this conclusion comes from the significantly anisotropic distributions of satellite galaxies (baryonic mass $<10^{8} M_{\odot}$ ) which orbit in the same direction around their hosting galaxies in disk-like structures which cannot be derived from dark-matter models. (2) The action of dynamical friction due to expansive and massive dark matter halos must be evident in the galaxy population. The evidence for dynamical friction is poor or even absent. Independently of this, the long history of failures of the SMoC have the likelihood that it describes the observed Universe to less than $10^{-4} \%$. The implication for fundamental physics is that exotic dark matter particles do not exist and that consequently effective gravitational physics on the scales of galaxies and beyond ought to be non-Newtonian/non-Einsteinian. An analysis of the kinematical data in galaxies shows them to be described in the weak-gravitational regime elegantly by scale-invariant dynamics, as discovered by Milgrom. The full classical regime of gravitation is effectively described by Milgromian dynamics. This leads to a natural emergence of the simple laws that galaxies are indeed observed to obey. Such success has not been forthcoming in the dark-matter-based models. Observations of stellar populations in galaxies suggest the galaxy-wide IMF, the IGIMF, to vary with star formation rate and that stochastic descriptions of star formation are inconsistent with the data. This requires a re-interpretation of the stellar-mass assembly in galaxies and thus of the accretion rates onto galaxies. A consequence of this understanding of galactic astrophysics is that most dwarf satellite galaxies are formed as tidal dwarf galaxies in galaxy-galaxy encounters, that they follow the mass-metallicity relation, that galactic mergers are rare, that galaxies immersed in external potentials are physically larger than isolated galaxies and that star-forming galaxies form a main sequence. Eight predictions are offered which will allow the concepts raised here to be tested. A very conservative, coldand warm-dark-matter-free cosmological model may be emerging from these considerations.
\end{abstract}

\section{Introduction}

$\mathrm{The}^{1}$ standard model of particle physics (SMoPP) allows the calculation and prediction of particles, their excited states and their interactions. Although it is based on many parameters the origin of which are unknown, the mathematical framework has been shown by many experiments to be an excellent model of the real elementary particles and their interactions. Experiments have not yet shown evidence for physics beyond the standard model $\left([1,2]^{2}\right)$.

The standard model of cosmology (SMoC) likewise allows the computation and prediction of galaxies, their perturbations

\footnotetext{
${ }^{1}$ This manuscript is part of a special issue whose topic is MOND: modified Newtonian dynamics.

${ }^{2}$ But see [3] for a possible indication of new physics at an energy scale $>1$ Tev and [4] for indications of physics beyond the standard model based on the proton radius puzzle.
}

Pavel Kroupa. Helmholtz-Institut für Strahlen- und Kernphysik, Nussallee 14-16, D-53115 Bonn, Germany, Institut für Astrophysik, University of Vienna, Austria.

E-mail for correspondence: pavel@ astro.uni-bonn.de and interactions. These computations are compared to observed galaxies and their distribution, but require the star-formation process to be understood at the level of time-dependent distribution functions that describe newly formed stellar populations.

The aim of this contribution is to perform two tests which challenge the SMoC. The two tests are based (i) on the dual dwarf galaxy theorem and (ii) on dynamical friction. Furthermore, this text attempts to collate what the empirical evidence implies for the astrophysics of galaxies, in terms of their dynamics and star-formation processes. The overall conclusion is that galaxies appear to be simple and computable systems.

Before introducing both, an outline of the $\mathrm{SMoC}$ is given in Sect. 2 to allow an appreciation of its structure and weaknesses. A description of the two different types of dwarf galaxies that ought to exist, primordial dwarfs and tidal dwarfs (e.g., [5]), is given in Sect. 3, before presenting the two tests falsifying the SMoC in Sect. 4. The robustness of the falsification is assessed in Sect. 5, based on the anisotropic distributions of galaxy satellites (e.g., [6-9]), on the mass-metallicity relation expected for tidal dwarfs, and on the various internal inconsistencies of the SMoC. The necessary replacement of Newtonian dynamics by Milgromian dynamics $[10,11]$ is presented in Sect. 6. The build-up of stellar populations in a dark-matter- 
free universe is discussed in Sect. 7. Here it is argued that observational evidence disfavors stochastic star formation, and with a proper understanding of the systematic variation of the galaxy-wide stellar initial mass function, galaxies also become much simpler than thought. Putting the evidence outlined here together, it may be possible that a highly conservative, Einsteinian cosmological model without cold or warm dark matter may have already been discovered (Sec. 8). Eight predictions inspired by this whole framework are presented in Sect. 9, and conclusions are drawn in Sect. 10. Appendix A lists the used acronyms, their meaning and first-time occurrence in this text.

\section{The Standard Model of Cosmology (SMoC)}

Fundamentally the SMoC is based on two assumptions: $H y$ pothesis $0 \mathrm{i}$ : Einstein's general relativistic field equation is valid and accounts for the space-time-matter coupling. Hypothesis Oii: All matter condensed just after the hot Big Bang (e.g. [12]). Both assumptions are supported by observational evidence (for example, general relativity has passed all tests in the Solar System and near neutron stars; hot Big Bang nucleosynthesis broadly explains the observed abundance patterns, [13-15]).

The computation of an expanding universe under hypotheses $\mathrm{O} i$ and Oii leads to a model that is far more structured and curved than the observed Universe. The Universe is observed to have a nearly perfectly flat geometry rather than being curved. The large-scale distribution of observed matter (galaxies) and the very high uniformity of the cosmic microwave background $(\mathrm{CMB})$ radiation imply the universe to be nearly homogeneous and isotropic ${ }^{3}$. Since there is no evidence for physics beyond the SMoPP in the observable Universe, it has also been deduced that all parts of the known Universe had to have been in causal contact. Introducing an augmentary hypothesis 1 , inflation, solves all of these problems at the prize that the physics underlying inflation is not understood. According to this hypothesis, the volume of the Universe inflated by a factor of about $10^{78}$ in about $10^{-33} \mathrm{sec}$. Evidence for the predicted inflationary gravitational waves may have been discovered (BICEP2, [16]), although the signal may also be due to the Galactic dust foreground $[17,18]$.

This resulting model based on hypothesis Oi, Oii and 1 can neither account for the observed rotation curves of galaxies nor does it form structures rapidly enough in comparison with the observed Universe. This sets limits on the amount of gravitating matter which can be present in the Universe, and normal (i.e. baryonic) matter (that is, matter, which is accounted for by the SMoPP) is observed to contribute significantly too little. Given historical precedents of successfully introducing new matter components (notably the prediction of the existence of the planet Neptune because of discrepancies between calculations and data for the planet Uranus; the prediction of the neutrino based on missing energy and momentum in the radioactive beta decay), a further augmentary hypothesis 2 was introduced, namely that unseen (dark) matter must exist to boost the matter density, to aid in structure formation and in an attempt to explain the constant-rotation-velocity curves of galaxies (for a review of dark matter see e.g. [19-21]). That galactic

\footnotetext{
${ }^{3}$ But see Fig. 1.
}

rotation curves indeed remain super-Keplerian and nearly flat at large radii was discovered by Rubin \& Ford (1970, [22]) and established by Bosma (1981, [23]). An early argument for the existence of dark halos was brought up by Ostriker \& Peebels (1973, [24]) who showed that for disk galaxies to be stable against bar formation their potential needs to be deepened. There are thus well motivated empirical astronomical arguments suggesting the presence of a significant dark matter component in galaxies. Bahcall \& Casertano (1985, [25]) argued that the dark halo profile and the baryonic profile need to be fine tuned in order to allow for the featureless transition observed between the inner, visible matter dominated rotation curve and the outer, dark halo dominated curve. This suggested that the dark halo may be made up of normal matter.

Possible dark matter candidates such as faint dwarf stars (e.g. [26]) and black holes were excluded [27] by the end of the 1980's. The fine-tuning pointed out by Bahcall \& Casertano [25] was used as an argument that cold molecular gas may be providing the dark component [28]. Observational limits on structure formation and nucleosynthesis however pose a challenge for this proposition, leaving only the possibility that dark matter (DM) be comprised of new elementary particles which do not interact significantly with normal matter except through gravitation [29]. This is consistent with the constraints on the density of baryonic matter provided by hot big bang nucleosynthesis. Broadly two categories are being considered: cold dark matter (CDM) for massive particles $(>10 \mathrm{keV})$ and warm dark matter (WDM) for light dark matter particles (of the order of 1-10 keV; axions being an exception as they are light but cold). Collectively these are referred to here as exotic dark matter particles. These are dissipationless ballistic particles that map out the phase space of the gravitational Newtonian potential they create. They cannot interact electromagnetically nor via the strong force as they need to decouple from normal matter before it recombines in order to form the gravitational seeds for structure formation. An excellent review of the present state-of-the art of the dark matter problem is provided by [21].

The dark-matter cosmological model [30,31], which is based on hypotheses $\mathrm{Oi}$, Oii, 1 and 2, was found to have a slower current expansion rate than the real Universe as measured using supernova of type Ia (SNIa) as standard candles [32-34]. This important discovery forced the introduction of hypothesis 3 which is a new phase of inflation driven by dark energy (for reviews see $[35,36]$ ). An undenyingly beautiful aspect of this finding is that dark energy can be identified readily with the constant $\Lambda$ in GR.

The resulting SMoC (e.g. [37], [38]), meaning hereon collectively the $\Lambda \mathrm{CDM}$ or $\Lambda \mathrm{WDM}$ models, has an energy density made up of about $75 \%$ dark energy, $20 \%$ exotic dark matter, and only about $5 \%$ baryonic matter. Since the Universe is known to be expanding issues of energy conservation arise, given that $\Lambda$ corresponds to a constant vacuum energy density [39]. [40] argues that the negative-pressure equation of state of the vacuum makes it a source of unlimited energy allowing any region to inflate arbitrarily at a constant energy density.

The SMoC has convincing aspects in the sense that the augmentary hypotheses can be incorporated into physics. For example, dark matter particles can be identified in extensions of 
the SMoPP (such as supersymmetry), and dark energy can be identified with Einstein's $\Lambda$. These are, however, not at present understood and have no independent observational foundation apart from the data that were used to argue for their introduction. Thus, there is neither evidence for dark matter particles from particle experiments [41], nor has experimental evidence for supersymmetry been found. Given the present-day nullevidence for physics beyond the SMoPP, despite the searches at the highest available energies with the LHC, theoretical approaches appear to be under crisis [42]. This is especially relevant for the putative existence of exotic dark matter particles. Diaferio (2008, [43]) reviews this situation and discusses the possible implications for gravitational physics. There is also a crisis in the concept of searching for dark matter particles within the framework of testing the SMoC. The case can be made that this is an ill-conceived scientific procedure because, as emphasized by McGaugh [44], the existence of dark matter particles can not be falsified by direct searches. Their existence cannot be falsified because in the event of null detections it can always be argued that the detection cross section lies below the instrumental threshold (see footnote 8 ). The model can therefore be criticized as being largely (to $95 \%$ in energy content) based on unknown physics and to not be falsifiable, and it may therefore be viewed as being unsatisfying, a view also suggested by [43]. Here I argue that the existence of cold or warm dark matter particles can be falsified by astronomical data.

One important caveat needs to be remembered: when Einstein formulated the theory of general relativity (GR, [45]), the data he had used indirectly as constraints were provided through Newton's empirically derived law of gravitation. This law is constrained in its validity to the scale of the Solar System only. Neither galaxies nor the dynamics of matter within them had been established in the 1910 s to what we know they are today (see [5] for a discussion of this discovery). Indeed, the "derivation" of Einstein's field equation appears rather heuristic and very much driven by Newtonian properties [46]. The application of GR to all scales of the Universe therefore constitutes an extrapolation by more than 5 orders of magnitude in acceleration and by 6 and more orders of magnitude in spatial extent. Such an extrapolation of an empirically derived law in the gradient of the interaction potential or in the range of the interaction bears the risk of not being correct.

The SMoC is based on a major extrapolation of the empirical pre-galactic foundation of the law of gravitation.

Perhaps this is one of the reasons why it had a torturous development path in the sense that the mathematical description usually led to failures such that it had to be repeatedly enriched by augmentary hypotheses beyond the original ones (hypothesis $0 \mathrm{i}$ and $0 \mathrm{ii}$ ). This could be viewed as the discovery of new physics in some cases, as long as independent experimental verification is attainable.

In this context it is known that Einstein's field equation is not unique (e.g. [46]) and generalizations have been developed ${ }^{4}$.

\footnotetext{
${ }^{4}$ One generalization of it which incorporates the correct dynamics in experimentally tested limits, particularly in the classical very weakfield limit avoiding the need for cold or warm dark matter, has been discovered and published as TeVeS in 2004 by Bekenstein [47, 48].
}

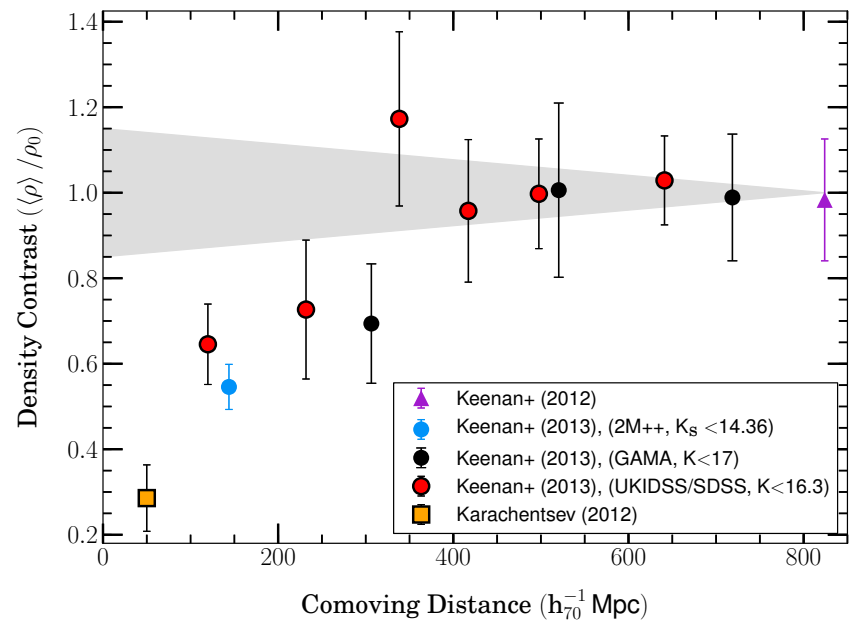

Fig. 1. The Karachentsev-Keenan under density of matter around the Sun (see Sec. 5.2). The baryonic matter density is plotted as a ratio to the average background density in the SMoC vs distance from the Sun to distances of about $800 h_{70}^{-1} \mathrm{Mpc}$ (redshift $z \approx 0.2$ ). Here the Hubble constant is assumed to be $H_{0}=70 \mathrm{~km} \mathrm{~s}^{1} \mathrm{Mpc}^{1}$ (i.e., $h_{70}=1$ ). The $\mathrm{SMoC}$, if it were a correct representation of the Universe, ought to have fluctuations smaller than about $10 \%$ on scales of $50-400 \mathrm{Mpc}$ ( [68], see also fig. 12 in [69], here indicated by the grey area). The observed under density appears to provide the right amount of local inhomogeneity to eliminate the need for dark energy within the SMoC. This figure was kindly prepared by F. Lüghausen.

The generalizations which are already known to account for the observed dynamics on galaxy scales without cold or warm dark matter need to pass all experimental tests.

Today the very major part of the scientific community understands the SMoC to be an excellent description of the observed Universe (e.g. [52,53] for an introduction). But some authors do acknowledge that significant tensions between the SMoC and the data exist $[11,20,54-66]$. Perhaps a worrying issue is that it is unclear whether it is a $\Lambda \mathrm{CDM}$ or a $\Lambda \mathrm{WDM}$ universe [67]. And, there appears to be a significant under-density of matter in the local Universe [68,69] (see also Fig. 1). The SNIa evidence for late-time acceleration, i.e. for dark energy, may disappear entirely if this under density is real [69]. Furthermore, the distribution of galaxies in the Local Volume does not correspond to what is expected from the SMoC in terms of the stratification of the type of galaxies in the Local Void and the Local Filament [63]. Also the baryonic to dark-matter-halo masses of dwarf galaxies in the SMoC do not agree with the observed values [70], and, given the importance of mergers in the SMoC, predictions for galaxy populations do not matchup with the observed populations [53,71,72]. Interesting is the argument by Matteucci [57] that the observationally deduced rapid and early formation times of elliptical galaxies and the associated down-sizing problem contradict the hierarchi-

Another generalization has been published in 2007 by Zlosnik [49] as an Aether vector field which couples with the space-time metric. Milgrom discovered a bimetric generalization in 2009 [50]. Nonlocal and pure-metric non-Einsteinian gravity models have also been investigated by Deffayet et al. (2014, [51]). 
cal merger-driven build-up of the galaxy population which is, however, the process implied by the SMoC [73-75]. The extreme correlation between the density of dark matter and that of baryons as evidenced by low-surface brightness galaxies has been used as an argument against the SMoC by McGaugh [55], and that observed disk galaxies are much simpler than expected in the $\mathrm{SMoC}$ has been demonstrated by Disney et al. [61]. Despite these problems many authors warn from discarding the model. A major argument fielded against such a step is that baryonic processes may influence the distribution of dark matter significantly [76]. A clear-cut falsification [5] would have major deep implications for physics.

Being fully aware that this goes against the majority opinion and against authority [37], here I show that the SMoC cannot be a correct model describing the Universe. A formal falsification of the SMoC can already today be deduced using two independent arguments: by applying the dual dwarf galaxy theorem and by the lack of dynamical friction. Each is discussed in turn in the following. Consistency checks are performed. Essentially, the argument excludes the existence of exotic dark matter particles, the existence of which are however the carrying pillar of the SMoC.

Reiterating from [5], "for a logical construction to be a scientific theory it has to be falsifiable. Otherwise predictions are not possible and the construction would not allow useful calculations." Furthermore,

UNIQUENESS AND FALSIFICATION: If a theory is found to be consistent with some data, then this does not prove that there is no other theory which has the same success. However, disagreement of a prediction with data, if verified, falsifies the theory.

A critical researcher may query his or her concept of what a successful theory ought to or ought not to achieve with the following Gedankenexperiment ${ }^{5}$ :

CHALLENGE FOR THEORY: Take a single isolated disk galaxy for which the distribution of baryonic matter has been mapped out with high resolution. Predict the rotation curve.

\section{The Types of Satellite Galaxies}

Before stating the dual dwarf galaxy theorem a brief review of the types of dwarf satellite galaxies which ought to exist is given. A dwarf galaxy is taken to be one with a baryonic mass $M \lesssim 10^{8} M_{\odot}$.

Concerning the definition of what a galaxy is, within the present context a discrimination criterion is needed which does not rely on dark matter. Using the median two-body relaxation time as a decision criterion is a possibility $[5,77,78]$ : all systems commonly referred to as galaxies have two-body relaxation times longer than a Hubble time, while all systems commonly referred to as star clusters have had the process of energy equipartition playing an important role over a Hubble time, and thus have two-body relaxation times shorter than a

\footnotetext{
${ }^{5}$ The word "predict" ie here meant to mean what it means, i.e. a calculation of a quantity or set of quantities before any observational data on these quantities exists.
}

Hubble time. This definition of a galaxy implies the time evolution of the stellar phase-space distribution function of such a self-gravitating system to be described by the collisionless Boltzmann equation (CBE). Star clusters require collisional dynamics methods such as the direct $N$-body method [79] to be employed to follow the time-evolving phase-space distribution of its stellar population.

GALAXIES VS STAR CLUSTERS: Galaxies are self-bound stellar-dynamical systems, the time-evolution of the stellar phase-space distribution functions of which are solutions of the CBE. This is synonymous to galaxies being systems which have median two-body relaxation times longer than a Hubble time. Star clusters are self-bound stellar-dynamical systems which have median two-body relaxation times shorter than a Hubble time. The time-evolution of a cluster's stellar phasespace distribution function is not a solution of the CBE, and is driven by the energy-equipartition process.

\subsection{Primordial dwarf galaxies (PDGs)}

A realistic cosmological theory includes a description of the formation of galaxies. And, in any theory there is likely to be a lower-mass limit below which dwarf galaxies do not form. As a Gedankenexperiment, in a dark-matter free cosmological model one might envision that the lowest-mass galaxy is given by the local Jeans mass in the expanding and cooling post-bigbang gas.

In the SMoC the exotic dark matter particles decouple from the baryons at a very early stage and form self-bound structures, the first dwarf dark matter halos. After recombination these accrete cooling baryons and set the scale of the lowestmass galaxy evident today subject to reionisation which may inhibit star formation [80]. The predictions are that major galaxies such as the Milky Way (MW) and Andromeda ought to have thousands of dark-matter sub-halos orbiting within the dark matter halo which surrounds them with a radius of about $250 \mathrm{kpc}$ as documented by a number of seminal contributions [81-84].

These satellite galaxies are typically distributed spheroidally about their host galaxy following the distribution of the host dark matter [85-88], because they have independent and uncorrelated accretion histories onto the growing host dark matter halo (see also Sec. 5.1.1 and 5.1.3). In particular [9] re-analyse all recently made claims in the literature concerning the theoretical three-dimensional distribution of primordial satellite galaxies within MW-type host dark matter halos confirming this theoretical spheroidal and highly non-disk-like distribution (see [89] for similar results).

In the SMoC PDGs are dominated by dark matter at all radii and thus have very large dynamical mass-to-light ratios $\left(M / L>10 M_{\odot} / L_{\odot}\right.$, taking from hereon $L$ to be the bolometric luminosity). Indeed, the dwarf spheroidal (dSph) and ultra-faint dwarf (UFD) galaxies found around the MW and Andromeda do have $M / L \approx 10-1000 M_{\odot} / L_{\odot}$ by observation when interpreting the data in terms of Newtonian dynamics [90].

Because the MW and Andromeda both have merely a few dozen satellite galaxies each (Figs. 2 and 3), a major tension between the SMoC and the real Universe is the satellite over prediction problem (popularly known as the missing satellite 
problem). A consensus has emerged in the community that this problem has been largely solved by various baryonic processes inhibiting the formation of PDGs in most low-mass dark matter halos, as is echoed in various research papers (e.g. [52, 97-100]). For example, [100] write about the observed faintest satellite galaxies of the MW, "there is now little doubt that these are the remnants of the galaxies born at high red-shifts in low-mass DM halos."

AVAILABLE FACTS: In the SMoC major galaxies such as the MW and Andromeda ought to have a large number (see Sec. 5.2) of satellite PDGs each with their own dark matter subhalo and each being brighter than a typical open cluster, but with a very large dynamical mass-to-light ratio. These satellite galaxies are typically distributed spheroidally about their host galaxy reflecting the three-dimensional structure of the hosting dark matter halo.

\subsection{Tidal dwarf galaxies (TDGs)}

When two gas-rich galaxies interact angular momentum and energy are redistributed throughout the interacting system. This involves a compensation of the temporarily increased binding energy by acceleration to larger velocities and therewith expulsion of part of the matter. Under certain conditions and if one or both interacting galaxies are disk galaxies the tidal material leaves in a kinematically cold (velocity dispersion of the pre-encounter disk) tidal tail. The tidal arm is then essentially the outward extension of a spiral pattern which is induced through the galaxy encounter. The gas in the tidal tail fragments into star clusters, star-cluster complexes and dwarfgalaxy-scale star-forming clumps, hereafter referred to as tidal dwarf galaxies (TDGs, [101, 102]). A noteworthy features is that the objects born in a tidal tail constitute highly-correlated structures in phase-space. A particularly beautiful example of this is the Hubble Space Telescope image of the Tadpole galaxy, evident in Fig. 4.

Experience shows that high-resolution simulations of galaxy interactions with gas often lead to the formation of TDGs (e.g. $[102,104-106]) .^{6}$ A noteworthy feature of TDG formation is that they and the star clusters form in tidal arms which constitute highly correlated structures in phase space. TDGs and star clusters formed in one tidal arm continue orbiting in the same direction around their host galaxy and form, for many orbital times, populations of objects that are aligned in a disklike structure around the host galaxy.

An important result from all these simulations is that TDGs form by containing very little exotic dark matter [5, 101, 109112]. Their potential wells are so shallow (corresponding to maximum circular velocities of about $v_{c} \lesssim 30 \mathrm{~km} / \mathrm{s}$ ) that the exotic dark matter particles which are virialised within the much more massive host dark matter halos $\left(v_{c}>100 \mathrm{~km} / \mathrm{s}\right)$ transgress

\footnotetext{
${ }^{6}$ Statements do exist in the literature according to which very special galaxy-galaxy encounters are needed to produce TDGs and that "a large fraction are certainly gravitationally unbound star-forming knots that will quickly dissolve". While the formation and survival of TDGs over Gyr time-spans is a pressing problem to study $[107,108]$, the rationalisation of these statements is not clear, given the already available high-resolution computational work, as discussed in this Sec. 3.2.
}

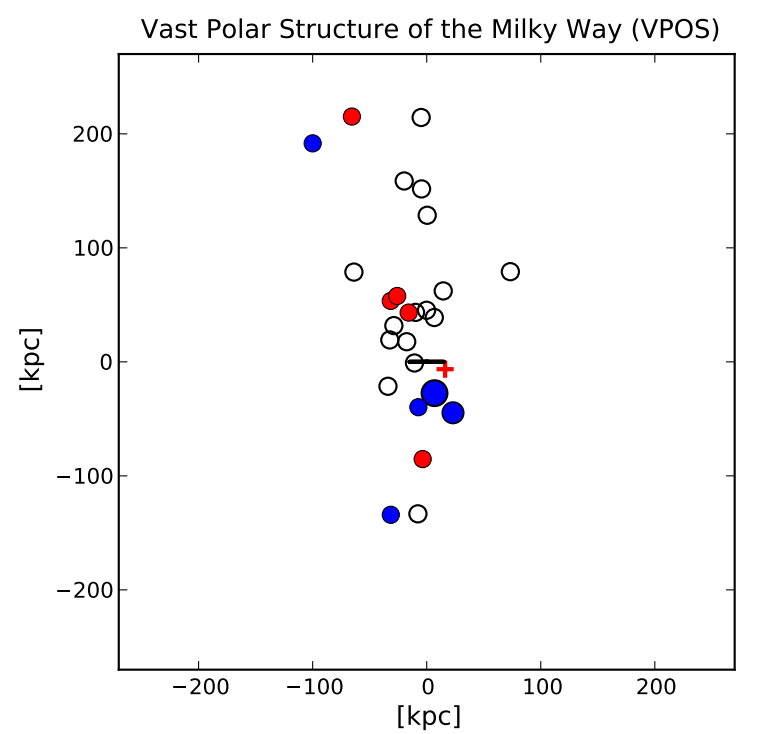

Fig. 2. The vast-polar structure (VPOS) or disk-of-satellites (DoS) of the MW seen edge-on (see Sec. 5.1.1.1)("VPOS-3 orientation", see [91]) such that the north pole direction of the Local Group and of the MW is along the y-axis towards the top (see fig. 6 in [91]). The face-on view is shown in fig. 2 in [92]. The MW disk is seen here edge-on and is shown as a $30 \mathrm{kpc}$ long thick black line at the centre of the figure. The filled circles and the cross are the eleven classical (brightest) dSph satellite galaxies. These have proper motion measurements such that estimates of their three-dimensional motions about the MW are available. Satellites colored in red are moving away from the observer who is situated at infinity such that Andromeda lies behind and to the left of the MW from this viewing direction. Open circles are ultra-faint dwarf (UFD) satellite galaxies for which proper motion measurements do not exist yet (see prediction 9.2 in Sec. 9). The Large and Small Magellanic Clouds are shown as the largest and intermediate sized blue circles, respectively. The red cross depicts Sagittarius, which is close to being on a perpendicular orbit to the VPOS and around the MW and thus orbits within the here-shown plane apart from a small component away from the observer. The counter-orbiting satellite in the north is one of the outermost satellites, Leo I, which is on a very radial orbit (and only has a small velocity component into the plane of the figure), while the one in the south may be a case which arises naturally if the satellite galaxies are TDGs: the ratio of the two counter-orbiting populations yields constraints on the MW-other-galaxy interaction which produced the tidal arms within which the TDGs formed [93]. The VPOS appears to be mass-segregated such that the most massive satellites are near its mid-plane (see Sec. 10.1.7 in [5]). The newly discovered satellite Crater or PSO J174.0675-10.8774 with absolute visual magnitude $M_{V} \approx-5.5[94,95]$ lies just to the lower right of the fourth open circle from the top and enhances the VPOS [96]. Andromeda also has a great plane of satellites which is discussed in Fig. 3. This figure was kindly provided by M. Pawlowski. 


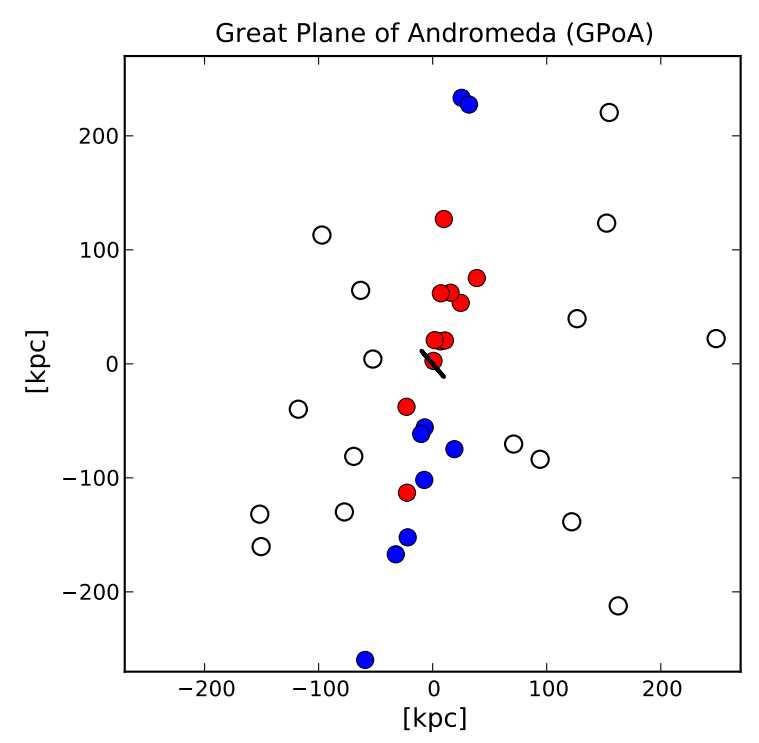

Fig. 3. The great plane of Andromeda (GPoA) or vast thin disk of satellites (see Sec. 5.1.1.2) confirmed by Ibata et al. [6]. This GPoA contains 19 and thus about half the satellite population of Andromeda, has a diameter of about $400 \mathrm{kpc}$ and a perpendicular scatter of about $14 \mathrm{kpc}$ and constitutes therewith an even more extreme example of a phase-space-correlated population of satellite galaxies than the whole VPOS of the MW (Fig. 2). The GPoA is also rotating mostly in one direction: in this rendition the observer is situated near the MW, the y-axis points towards MW-north and the satellites moving away in Andromeda's rest-frame are red, while the ones moving towards the MW are blue. The other satellite galaxies not in this kinematically correlated structure are shown as open circles. Note that the GPoA and the VPOS (Fig. 2) are rotating in the same sense, although the two disks are inclined by about $38 \mathrm{deg}$ (fig. 16 in [91]), whereby the GPoA is seen edge-on from the MW. Both the GPoA and the VPOS are nearly perpendicular to the MW disk. This figure was kindly provided by M. Pawlowski.

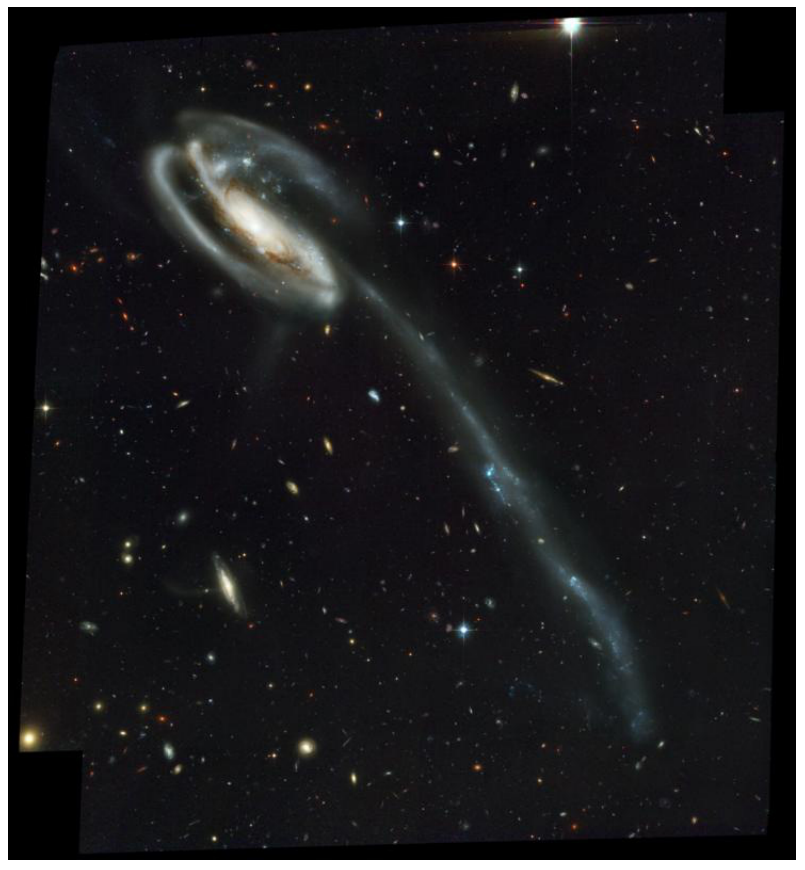

Fig. 4. The tadpole galaxy (UGC 10214) is a MW-class system and lies at a distance of about $129 \mathrm{Mpc}$. Probably a more compact galaxy crossed in front of the Tadpole Galaxy (from left to right) and orbited around the Tadpole to now lie about $90 \mathrm{kpc}$ behind the Tadpole. During this process a tidal arm with a length of about $86 \mathrm{kpc}$ was drawn out. This tidal tail shows regions of star formation in star clusters, many of which are clustered within two low-mass TDGs. This image [103] captures the thinness of tidal tails, the star formation within them and the "lego-principle", according to which stars form in embedded star clusters which are the basic building blocks of galaxies (see Fig. 10 for a close-up and Sec. 7). This image shows a region spanning about $105 \mathrm{kpc} \times 99 \mathrm{kpc}($ vertical $\mathrm{x}$ horizontal). Image credit: NASA, H. Ford (JHU), G. Illingworth (UCSC/LO), M. Clampin (STScI), G. Hartig (STScI), the ACS Science Team, and ESA. Publication with kind permission by $\mathrm{H}$. Ford. 
without being captured. Thus, any rotation curve of matter within a TDG or any dynamical $M / L$ ratio measurement of a TDG can only be due to a baryonic matter content assuming the TDG to be in virial equilibrium and the SMoC to be valid [101]. For pressure-supported (spheroidal) TDGs (old TDGs that have lost their gas) repeated tidally induced and thus non-uniform depletion of their phase space [113] over many orbits leads to quasi-stable solutions that feign domination by dark matter without it being present $[111,114,115]$.

The process of TDG formation is well documented observationally in the present-day Universe ( [112], for a collation of available observational data see [116]). At large redshift, galaxies are known to appear perturbed and are most likely interacting at a significantly larger rate than in the present-day Universe, although a bias exists for detecting such systems because they are likely to be brighter with interesting morphologies (see [117] for a related discussion). At distant cosmological epochs galaxies would have been richer in gas. The formation of TDGs would have been much more frequent then. Indeed, the systematic numerical study Wetzstein et al. [104] has demonstrated that the number of formed TDGs increases with increasing gas fraction. Today a large fraction if not all of the dwarf satellite population may be composed of ancient and in some cases young TDGs even assuming the SMoC to be valid. This has been calculated assuming a merger tree consistent with the SMoC by [118], and is also suggested by the correspondence between structural parameters of dwarf earlytype galaxies and observed TDGs [116]. It is also supported by the observational finding that dwarf elliptical (dE) galaxies have stellar mass-to-light ratios within their optical regions such that their DM content is not significant $[119,120]$.

However, this conjecture that most if not all dwarf satellite galaxies are TDGs needs to be verified with high-resolution simulations of TDG formation and evolution, as is now being performed in the Vienna group by Plöckinger, Recchi and Hensler [107]. [108] provide significant observational evidence that TDGs survive for at least a few Gyr.

TDGs would not constitute a major population of satellite galaxies today if TDGs dissolve after they form. TDGs may dissolve in three ways: (a) They may "fall-back" on to their host galaxy. (b) They may become unbound as a result of the star-formation within them driving out the matter that binds them. (c) If they survive their formation they may be destroyed by tidal forces from their host galaxy.

Case (a) is ruled out because tidal material cannot "fall back" on its host galaxy, since orbital angular momentum is conserved and since TDGs are likely to form and survive only on orbits with large semi-major axes ( $\gtrsim 50 \mathrm{kpc}$ ). A TDG may be tidally shocked and as a result it may dissolve if it is on a highly radial orbit. These are rare cases though. Case (b) has been shown to not be the case by detailed simulations of the first few hundred Myr of TDG evolution including realistic star-formation and feedback descriptions by Recchi et al. [121] and Plöckinger et al. [107]. More simulation work is necessary though to ascertain the true life-times of TDGs, and the observation of a few Gyr old TDGs by [108] suggests survival not to be a problem. Case (c) has been shown, by long-term highresolution simulations, to not be likely either $[111,114,115]$. Observational evidence supports these theoretical results that TDGs do not dissolve upon formation (e.g. [122, 123]).
AVAILABLE FACTS: TDG formation has been common over cosmological times. They cannot contain significant amounts of exotic dark matter particles and they form in phase-spacecorrelated structures (i.e. in tidal tails). Available work suggests TDGs survive for many Gyr. It is not known yet which fraction of satellite galaxies are ancient TDGs. TDGs and star clusters that formed in one tidal tail form highly-correlated structures in phase-space.

\subsection{The dual dwarf galaxy theorem}

It follows that in any cosmological theory in which galaxies interact, the following is true:

THE DUAL DWARF GALAXY THEOREM [5]: In any realistic cosmological model, there exist two types of dwarf galaxies: Type A dwarf galaxies are PDGs that formed above a low-mass threshold given by the ambient physical conditions. Type B dwarf galaxies are TDGs.

\section{Testing the SMoC}

The SMoC can be tested with astronomical data using two unavoidable consequences of its underlying hypotheses. The tests are discussed in Sec. 4.1 and 4.2.

\subsection{TEST1: The dual dwarf galaxy theorem and the SMoC}

Assuming the SMoC were a correct description of the real Universe, then dwarfs of Type A would be dark-matter dominated and would thus constitute dwarf galaxies which formed in dark matter halos with masses that are significantly larger than their baryonic masses [70, 124]. Type B dwarfs on the other hand would have stellar/baryonic dynamical mass-to-light ratios $\left(M / L \lesssim 5 M_{\odot} / L_{\odot}\right)$ and would have formed through self-regulated star-formation in a self-regulated potential without a dark matter halo [104, 107, 121].

LOGICAL CONSEQUENCE OF THE DUAL DWARF GALAXY THEOREM [5]: If the SMoC is true then type A and type B dwarfs must have significantly different dynamical $M / L$ values and different morphological properties.

LOGICAL INVERSE OF THE DUAL DWARF GALAXY THEOREM [5]: If type B dwarfs cannot be distinguished dynamically from type $\mathrm{A}$ dwarfs (i.e. if type $\mathrm{B}$ dwarfs = type $\mathrm{A}$ dwarfs) then the SMoC is falsified.

This can be tested with two dual dwarf galaxy (DDG) tests:

\subsubsection{DDG Test I (late-type dwarf galaxies)}

If the SMoC is true then the baryonic Tully-Fisher relation (BTFR, [125]) is defined by the dark matter halos within which the luminous galaxies reside, because the circular velocity at the edge of or outside of the optical galaxy is determined for type A dwarfs by the dominating dark matter component. This is well established, given the observed high dynamical massto-light ratios of dwarf galaxies (deduced by applying Newtonian dynamics in the analysis of the data). Virialised type B 
dwarfs therefore must lie off the BTFR by having circular velocities which are a factor $>3$ smaller at the same galaxy luminosity than a type A dwarf assuming the conservative lower value for the dynamical $M / L$ ratio of $10 M_{\odot} / L_{\odot}$. Observations have shown three TDGs, for which rotation curves exist, to lie very close to the BTFR [126]. This is shown in Fig. 5. One TDG could lie close to the BTFR by chance if it is accreting gas such that the velocity field does not correspond to a virialised self-gravitating object. But that three TDGs which have been observed to have well-behaved rotation curves lie about equally close to the BTFR is unlikely to be chance. Cold molecular gas in the outer regions of the TDGs [122] cannot be the reason for the super-Keplerian rotation curves because this would require significant fine-tuning. That is, each TDG would have to have just the correct amount of cold molecular gas at the right radii in its outer regions to place it near the BTFR which is defined by the rotation velocity of the dark matter halo of the normal dwarf galaxies.

Result: Since by observation type B dwarfs = type A dwarfs in terms of the BTFR this falsifies the dual dwarf galaxy theorem under the assumption that the $\mathrm{SMoC}$ is true. Thus the $\mathrm{SMoC}$ is false. Note that this deduction is not sensitive to the survival of TDGs, because observed TDGs lie on the BTFR. Should they dissolve in the future would not impact this finding.

\subsubsection{DDG Test II (early-type dwarf galaxies)}

Consider now pressure-supported dwarf galaxies, that is, $\mathrm{dE}$ galaxies. Type A dwarfs reside in dark matter halos significantly more massive by a factor of 10 or more than their baryonic component [70,124]. Thus, either type B dwarfs at the same baryonic mass as a type A dwarf must have a velocity dispersion which is a factor 3 or more smaller than that of the type A dwarf (for the same effective radius), or the effective radius of the type $\mathrm{B}$ dwarf must be smaller by a factor of ten or more than the type A dwarf for the same velocity dispersion. However, the comparison of data on known TDGs with those of $\mathrm{dE}$ galaxies shows them to be indistinguishable in terms of their radius-mass relation, $\mathrm{R}(\mathrm{M}) \mathrm{R}$ [116].

Result: Since by observation the $\mathrm{R}(\mathrm{M}) \mathrm{R}$ of type $\mathrm{B}$ dwarfs $=$ the $\mathrm{R}(\mathrm{M}) \mathrm{R}$ of type A dwarfs this falsifies the dual dwarf galaxy theorem if the SMoC were true. Thus the SMoC is false. Note that this deduction is not sensitive to the survival of TDGs, because observed TDGs lie on the same radius-mass relation as the putative PDGs. Should they dissolve in the future would not impact this finding.

\subsection{TEST2: Dynamical friction}

If the SMoC is true then a satellite galaxy, be it of type A or B, suffers orbital decay due to Chandrasekhar dynamical friction within the dark matter halo of its host. An observational signature of orbital shrinkage of a satellite galaxy over time at a rate consistent with the dark halos of the $\mathrm{SMoC}$ would thus be a smoking gun for the existence of exotic dark matter particles. If galaxies are embedded in massive halos of exotic dark matter particles then they merge whenever encounters occur with galaxy-galaxy separations smaller than the sum of the virial radii of the dark halos (about $500 \mathrm{kpc}$ for the MW and

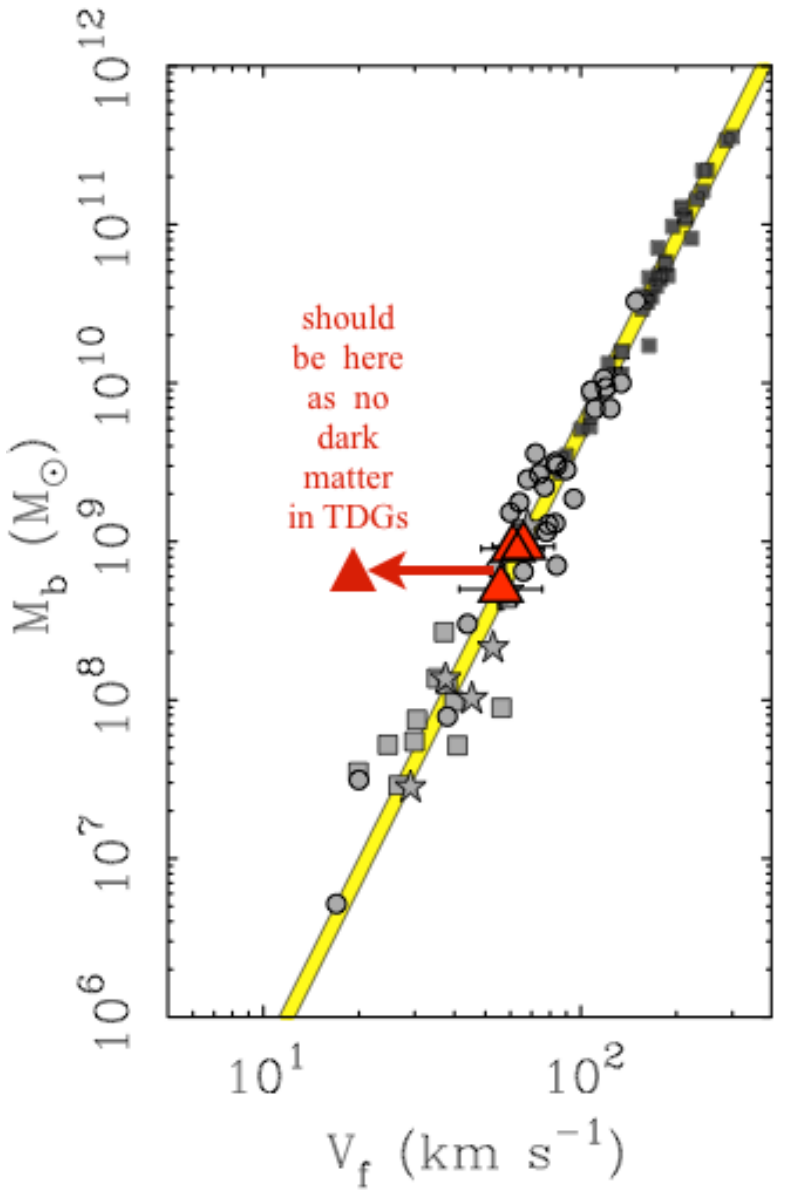

Fig. 5. The baryonic Tully-Fisher relation (BTFR) of late-type galaxies. The grey symbols are the total observed baryonic mass (stars plus gas) and observed rotation velocity $\left(v_{c} \equiv V_{f}\right)$ as collated and explained by McGaugh [125]. The three TDGs are plotted as red triangles. The red arrow points to the circular velocity at the outer radius of about $5 \mathrm{kpc}$ they ought to have in this diagramme if they were virialised rotationally supported dwarfs of type B without dark matter and in Newtonian gravity (see [122] for the rotation curves). The linear (yellow) regime is the BTFR (eq. 7). The width of the band represents the one-sigma uncertainty in the parameter $a_{0} \approx 1.21 \times 10^{-10} \mathrm{~m} \mathrm{~s}^{-2}$ obtained from detailed fits to the rotation curves of disk galaxies by [127]. 
Andromeda, for example) and with relative velocities comparable to the virial velocity dispersion of the dark halos (a few hundred $\mathrm{km} / \mathrm{s}$ for the MW and Andromeda, for example) or less. The merging times are comparable to the crossing times of the dominant dark matter halo (a few Gyr).

Some observational constraints on this issue are already available:

\subsubsection{The Sagittarius (Sgr) satellite galaxy of the MW}

Attempts at fitting the large number of observational constraints on the Sgr streams which are available over the past three orbits of the satellite have used models in which Sgr has a stellar mass of about $10^{8}-10^{9} M_{\odot}$ but no or only a minor dark matter halo [128-134]. Models without a dark matter halo may lead to better agreement with the observations [135].

If the SMoC were true, then a type A dwarf of baryonic mass $M=10^{8} M_{\odot}$ ought to have a dark matter halo mass of about $M_{\mathrm{DM}} \approx 10^{10} M_{\odot}$, while $M=10^{9} M_{\odot}$ would imply $M_{\mathrm{DM}} \approx 10^{11} M_{\odot}[70,124]$.

In models in which Sgr is assumed to have a dark matter halo it would have had to have fallen-in to the MW dark matter halo about 2.5 Gyr ago with a first peri-center passage about 1.4 to 2 Gyr ago [136]. But more work needs to be done in order to find an improved match to the observational constraints available for the Sgr streams. An analytical estimate of the orbital shrinkage time until merging of Sgr with the center of the MW leads to merging times of a few Gyr in agreement with the above result ${ }^{7}$. An alternative model exists in which Sgr was scattered onto its current orbit in an encounter near apogalacticon with the Magellanic Clouds 2-3 Gyr ago [139], so that Sgr is not convincing evidence for dynamical friction.

\subsubsection{Other $M W$ satellite galaxies}

Assume the satellite galaxies Fornax, Sculptor, Ursa Minor and Carina, which have measured proper motions, are of type A such that they have their own dark matter halos. By explicitly taking into account dynamical friction [140] have shown that acceptable orbits, which are also consistent with their ages and distribution in the disk-of-satellites or vast polar structure [8], appear to be non-existing. The problem lies in the satellites all needing to walk a tight-rope by not merging with the MW due to dynamical friction and not being completely dissolved by Galactic tides but loosing just enough dark matter halo mass to end up on their observed present long-period orbits.

\footnotetext{
${ }^{7}$ The dynamical friction time scale, eq. 7-26 in [137], is used with the following assumptions and results: the maximum impact parameter is about the extent of the Sgr dark matter halo taking this to be $b_{\max }=100 \mathrm{kpc}$ [138] and with a circular velocity $v_{c}=220 \mathrm{~km} / \mathrm{s}$ for the MW dark matter halo. If the dark matter halo of Sgr has a mass $M_{\mathrm{Sgr}}=10^{11} M_{\odot}$ then the friction time-scale is $t_{\text {fric }}=$ $2.5 \mathrm{Gyr}$ if Sgr began on a circular orbit at a Galactocentric distance $r_{i}=100 \mathrm{kpc}$. If the dark matter halo of Sgr has a mass $M_{\mathrm{Sgr}}=$ $10^{10} M_{\odot}$ then the friction time-scale is $t_{\text {fric }}=3.3 \mathrm{Gyr}$ if Sgr began on a circular orbit at a Galactocentric distance $r_{i}=50 \mathrm{kpc}$. For $b_{\max }=25 \mathrm{kpc}$ and $M_{\mathrm{Sgr}}=10^{9} M_{\odot}$ then $t_{\text {fric }}=6.5 \mathrm{Gyr}$ if Sgr began on a circular orbit at a Galactocentric distance $r_{i}=25 \mathrm{kpc}$.
}

\subsubsection{The M81 group}

The M81 group of galaxies consists of a MW-type primary with a few major star-forming satellite galaxies and strong evidence for past interactions between the galaxies in the form of pronounced gas bridges. Attempts at modelling this system with live and self-gravitating components including dark matter halos appear to have lead to null results: solutions have not been possible because the system merges within about a crossing time [141, 142]. Solutions are available, but without dynamical friction on the dark matter halos ( [142], see also [143]). No more-recent computational work is available. The "hot potato" was dropped, it seems.

\subsubsection{Merger-driven evolution of galaxy populations}

According to Barnes (1998, [144]) "Interacting galaxies are well-understood in terms of the effects of gravity on stars and dark matter." This is due to our excellent understanding of Newtonian dynamics and the collisionless nature of stars and of dark matter particles. Galaxies of similar masses merge within 0.5 to $3 \mathrm{Gyr}$ [145]. It has indeed become very popular in the community to refer to mergers or post-mergers when galaxies-in-interaction or galaxies-past-interaction are discussed, respectively. But galaxies-in-interaction are merely interacting galaxies, and "post-mergers" may just be galaxies that have had a strong encounter with another galaxy a few Gyr in the past whereby matter may have been transferred from one to the other via the tidal arms drawn out during the encounter (e.g. [146]). If two galaxies have a relatively close encounter with a relative pre-encounter velocity of $300 \mathrm{~km} / \mathrm{s}$ then they will have separated by $300 \mathrm{kpc}$ by one Gyr or nearly one Mpc after three Gyr, unless they merge.

Where does the implicit wide-spread understanding that galaxies merge whenever they interact come from? To the best of my knowledge this concept can be traced back to Toomre (1977, [147]) who interprets a few images of interacting galaxies as galaxies that are in the process of merging, and he speculates that elliptical galaxies form from such mergers. Today it is known that this is not the case (see below in this Section). Toomre [147] writes "These merger hopes would all be in vain, of course, if the severe kind of dynamical friction which they seem to require proved simply to be unattainable." In this influential paper, Toomre is worried that in order to get the observed tidal tail morphologies, off-center encounters are needed: "the big worry remains that the strength of braking may drop off too rapidly with increasing impact parameter or miss distance, as one seeks circumstances that will also permit the manufacture of tails of the sort summarized in Table I. . . . , it seems the center of one galaxy needs to impact the other system no farther out than about the $1 / 2$ or $3 / 4$-mass radius, lest the rapidity of their sinking cease to be impressive. . . . In principle at least, one can always embed them, prior ro any fateful encounter, within some appreciably larger and more massive systems like the much-debated extensive halos." This is a remarkable deduction supporting the evidence for DM, if galaxies merge. But is it true?

If the SMoC were true then a typical galaxy and its dark halo acquire their masses through a sequence of mergers driven by dynamical friction [73-75]. The galaxy population at lowredshift should be a result of this process. [72] confront semianalytical and semi-empirical models with observational data 
on low-redshift galaxies finding significant discrepancies, and in particular, that the observational data favor long-dynamical friction time-scales. This is qualitatively consistent with the conclusion by [148] and [149] that too many ( $>50 \%, 94 \%$ according to [150]) of all late-type galaxies (with baryonic mass $\gtrsim 10^{10} M_{\odot}$ ) do not have a classical bulge, being at odds with the merging-dominated growth history of galaxies if the SMoC were true.

It is important to re-emphasize at this point that the profuse merging behavior of galaxies in the SMoC is entirely the result of the assumed existence of exotic dark matter particles which form the dark halo potentials within which the galaxies exist.

The angular-momentum problem of late-type galaxies is a consequence of this same issue, because angular momentum is removed in the highly dissipative infall into the deep potential wells of the dark matter halos of gas streams (e.g. [53] and references therein). That the haphazard or stochastic process of galaxy mergers indeed appears not to be an important process in establishing the present-day galaxy population is also found by Speagle et al. (2014, [151]): late-type galaxies lie on a main sequence with a surprisingly small spread (Sec. 7.7). Galaxies appear to evolve in-step over cosmic time such that, approximately, their star-formation rates (SFRs) are proportional to their stellar masses.

According to the survey of galaxies with absolute magnitude in the $J$ band brighter than -20.3 (stellar mass $\gtrsim 1.5 \times$ $10^{10} M_{\odot}$ ) by [152], $\gtrsim 90 \%$ of all galaxies are late-type starforming galaxies, while only $3 \%$ are elliptical galaxies (see their fig.5), being completely in accord with the well-known previous results on galaxy populations (e.g. fig. 4.14 in Binney \& Merrifield 1998, [153]). This population mixture exists also about 6 Gyr ago [152]. A major challenge remains to understand this population mixture in the SMoC within which galaxies evolve and grow through a sequence of many mergers of their dark matter halos. [154] emphasize this tension between observed galaxies and the $\mathrm{SMoC}$ and argue that galactic disks may be rebuilt after significant mergers implying that half of all disk galaxies ought to have disks younger than 9 Gyr. This nevertheless does not solve the problem that more than half of all disk galaxies with circular velocity $>150 \mathrm{~km} / \mathrm{s}$ have no classical bulge [149], while [150] deduce $94 \%$ of their disk galaxy sample to not have a classical bulge. The evidence of early formation times at high redshift and short $(<2 \mathrm{Gyr})$ formation time-scales of elliptical galaxies also contradicts the mergerdriven build-up over time of the galaxy population [57]. [155] confirm that elliptical galaxies cannot have been forming from disk galaxy mergers. It is noted here, in advance of the discussion below, that at any redshift galaxies with peculiar morphologies may merely be interacting rather than merging, as mergers are deduced or required only in the framework of the SMoC.

The postulate that elliptical galaxies form rapidly within the $\mathrm{SMoC}$ in an early burst together with dry (i.e. gas-free) mergers at later times (to be consistent with the merger-driven buildup of dark halos which continues for the majority of dark matter halos over cosmic time) may be compromised in view of the majority of the galaxy population with baryonic masses larger than about $10^{10} M_{\odot}$ not being dry but being late-type disk galaxies containing significant amounts of gas. In other words, the population of to-merge dry galaxies does not appear to exist, unless less massive galaxies are involved mostly. But the not-yet-merged large number of dry dwarf galaxies with stellar population ages and chemical properties corresponding to those of the elliptical hosts are not evident in observations.

The postulate that disk galaxies only occupy dark matter halos which do not have a major merger after the formation of their thin disks is incompatible with the majority of dark halos having such mergers and the great majority of galaxies being disk galaxies, as noted above. As discussed in [156], SMoC simulations have shown that over the last $10 \mathrm{Gyr}$ about $95 \%$ of MW-mass-scale dark matter halos with a mass of $\approx 10^{12} M_{\odot}$ have undergone a minor merger by accreting a sub-halo with mass $>5 \times 10^{10} M_{\odot}$, and $70 \%$ of them have accreted a subhalo with mass $>10^{11} M_{\odot}$ [74]. According to [75] $69 \%$ of such halos have major mergers since $\mathrm{z}=3$ (11-12 Gyr ago) and $31 \%$ have major mergers in the past 7-8 Gyr. Mergers are therefore common for MW-scale halos in the SMoC. The simulations have also shown that major mergers (with equal mass galaxy pairs) disrupt disks completely and that the remnants of such mergers become early-type galaxies (elliptical galaxies or bulge-dominated galaxies [157]). Minor mergers (with a mass ratio $10: 1$ ) also lead to growth of the bulge and thickness of the disc (e.g. [158-160]). Thus, the very large fraction of observed bulge-less disc galaxies and disk-dominated galaxies ( $70 \%$ in edge-on disk galaxies) is inconsistent with the high incidence $(>70 \%)$ of significant mergers, a point also emphasized by [149] (see also [150]).

More recently, a series of new models using smoothed-particle hydrodynamics (SPH) and moving-mesh simulations [161-163] are stated to reproduce realistic disk galaxies if the host dark matter halo has a quiet merger history, that is, if it undergoes no major merger after redshift $z>1$ or 3. For example, [164] apply their new hydrodynamic algorithm AREPO5, which relies on a moving unstructured Voronoi tessellation together with a finite volume approach. They explicitly exclude one of their models at redshift $z=0$ because it is involved in an encounter with another model galaxy such that the disks are significantly perturbed. The rotation curves of the gas component of their model disk galaxies show significant drops at galactocentric radii beyond about $10 \mathrm{kpc}$ in significant disagreement with the observed HI rotation curves of disk galaxies which extend as far as a tracer can be detected and often to $50 \mathrm{kpc}$ and beyond (see e.g. the review by [11]). Indeed, [156] show that all disk galaxies hitherto modelled in the $\mathrm{SMoC}$ fail to reproduce the empirical mass-discrepancy-acceleration correlation (Figs. 6 and 7) and thus do not constitute adequate models of galaxy formation and evolution.

In the above simulations of the formation of disk galaxies, no mergers with a mass ratio of the sub-structure and host galaxy larger than 1: 10 are allowed at low redshift. These authors have thus, essentially, rediscovered the models by Samland \& Gerhard (2003, [166]) who demonstrated that disk galaxies can be grown in growing dark matter halos without mergers. But such galaxies are selected from the unlikely fraction of MW-scale halos with a quiet merging history in SMoC simulations.

Using the same AREPO5 code, Vogelsberger et al. (2014, [167]) report the hitherto largest full-scale cosmological simulation finding a "reasonable population of ellipticals and spi- 


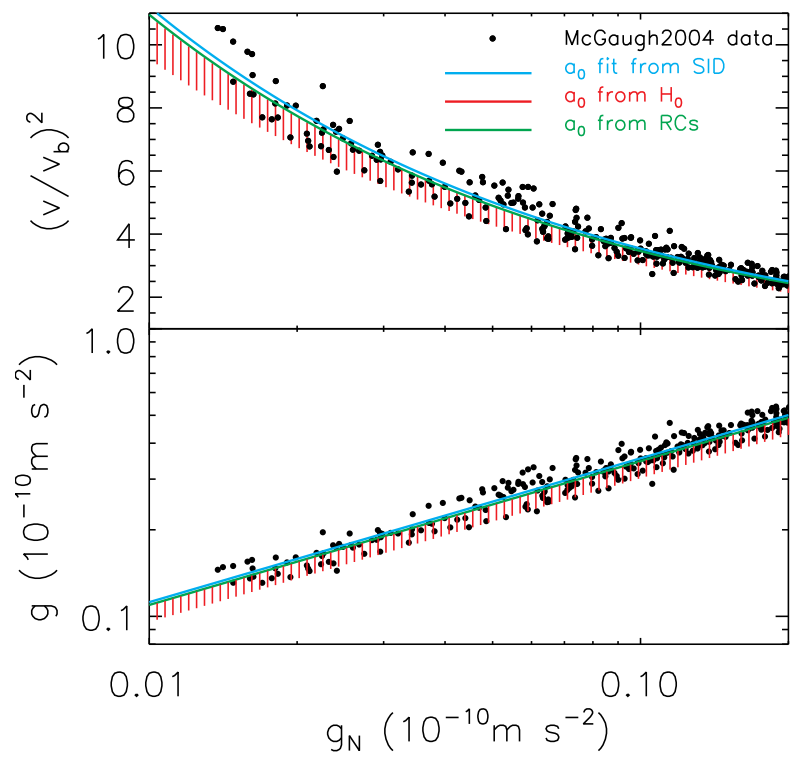

Fig. 6. Observational data (black dots, [165]) confirm that galaxies obey the MDA relation (eq. 8 , in this figure $\left.v \equiv v_{c}, v_{b} \equiv v_{c, b}\right)$. Upper panel: The MDA relation (eq. 8) is plotted for different values of Milgrom's constant $a_{0}$ as derived from independent sources (i.e. not from the black dots shown here), and as obtained from fitting the relation to the data (upper most solid cyan curve: $\left.a_{0}=(1.24 \pm 0.03) \times 10^{-10} \mathrm{~m} \mathrm{~s}^{-2}\right)$. The red shaded region is for $a_{0}$ according to eq. 9 for $H_{0}=76.4 \mathrm{~km} \mathrm{~s}^{1} \mathrm{Mpc}^{1}$ (upper limit) and $H_{0}=66.1 \mathrm{~km} \mathrm{~s}^{1} \mathrm{Mpc}^{1}$ (lower limit). The green curve is for $a_{0}=1.21 \times 10^{-10} \mathrm{~m} \mathrm{~s}^{-2}$ obtained by [127] by fitting rotation curves of disk galaxies. Lower panel: The true acceleration for a circular orbit, $g$, is plotted against the Newtonian acceleration, $g_{\mathrm{N}}$, computed from the baryonic matter content of the galaxy. The lines show eq. 5 for the different values of $a_{0}$ as in the upper panel. From [156].

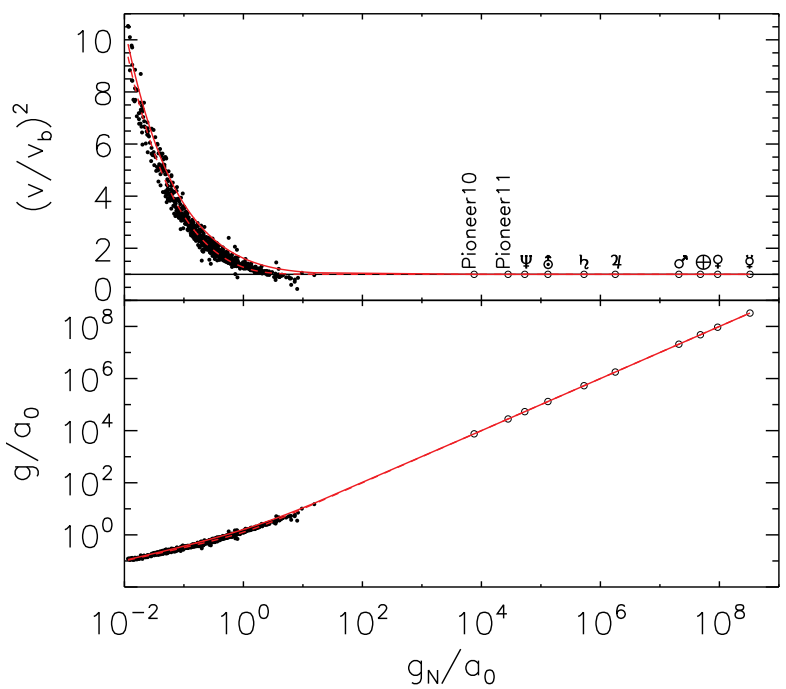

Fig. 7. The role of the function $\mu$ (eq. 14). Both panels are as in Fig. 6 but for the entire range of classical accelerations, including the Solar System objects collated by [11]. The solid red line is for the "simple" $\mu$-function and the dashed line for the "standard" $\mu$-function (both lines being very close to another). See [11] for details. From [156].

rals". This work is a milestone in simulating and visualizing structure formation down to galaxies with a stellar mass $M_{*} \approx 10^{9} M_{\odot}$ in the Universe assuming the SMoC to hold. The Illustris project [168] provides an on-line galaxy observatory. The computed population of galaxies results from mergers of smaller dark matter halos and awaits detailed studies in terms of their morphological types, their rotation curves and their location with respect to the empirical baryonic TullyFisher relation (Fig. 4.1.1). The authors do not address the major problems that have arisen in testing the SMoC on real data and their implementation of the IMF can be challenged (Sec. 7). Their simulated galaxies with low star-formation rates contain about $52 \%$ of all the stellar mass which is in galaxies more massive than $M_{*}=10^{9} M_{\odot}$. The authors state that this agrees well with observations, but according to [152] (see also e.g. fig. 4.14 in [153]) $\gtrsim 90 \%$ of all galaxies with $M_{*} \gtrsim 10^{10} M_{\odot}$ today and 6 Gyr ago are late-type star-forming galaxies. This may suggest a tension between the simulated and the real Universe in conformity with the previously known angular momentum problem. But the authors [167] suggest that the problem of forming Milky-Way-type galaxies has now been overcome: "The fact that our calculation naturally produces a morphological mix of realistic disk galaxies coexisting with a population of ellipticals resolves this long-standing issue. It also shows that previous futile attempts to achieve this were not due to an inherent flaw of the LCDM paradigm, but rather due to limitations of numerical algorithms and physical modelling." This appears to contradict the inability to form MW-type disk galaxies within the SMoC by the most recent work of another team [169]. This friction between the two SMoC models is not explained.

Another criticism that may be stated along the lines of Sec. 5.1.3 is that in [168] the Illustris model galaxies are close to the BTFR, while a previous recent study [71] cannot reproduce 
the BTFR within the SMoC. Vogelsberger et al. [168] do not discuss this discrepancy nor is [71] cited by them. A discussion as to why the Illustris models are closer to the BTFR than the Lu et al. models [71] would have been appropriate in order to clarify why both SMoC models disagree with each other. More thorough and independent comparisons between observed galaxies and the simulated ones await the publication of the simulated-galaxy catalogue. A perhaps major issue with the Illustris modelling is that it employs "a kinetic stellar feedback scheme, where the stellar wind velocity is scaled with the local halo environment $\left(3.7 \sigma_{\mathrm{DM}}^{1 \mathrm{D}}\right.$, where $\sigma_{\mathrm{DM}}^{1 \mathrm{D}}$ is the local onedimensional DM velocity dispersion", [167], p. 3 in [168]). In effect, the Illustris models assume local baryonic processes to couple closely to the entire DM halo. But the exotic DM particles do not interact electromagnetically with baryonic matter by construction of the SMoC. Neither the SMoPP includes such a coupling nor is there experimental evidence for such a coupling. This may thus be considered as being an unphysical assumption, these models being internally inconsistent at a fundamental level. Nevertheless, such models are valuable for gaining a deeper understanding of hypotheses that may be required to establish the galaxy population.

Collecting the above important work on the formation of elliptical galaxies and disk galaxies the following statement can be made with confidence:

The SMoC And The GAlaxy Population: The SMoC is untenable as a framework for galaxy formation:

Elliptical galaxies: must form quickly early on and require dry mergers to grow further and to be consistent with the merging history of the SMoC. This postulates a large population of dry galaxies which contradicts the observation that 6 Gyr ago only about $4 \%$ of all galaxies more massive than $1.5 \times 10^{10} M_{\odot}$ are ellipticals with this fraction not evolving since.

Disk galaxies: must form in dark matter halos with a quiet merging history. Even then the model disks have rotation curves not in agreement with observed ones. The required quiet merging history is appropriate for a minority of all host halos but this contradicts the observation that from 6 Gyr ago the very major majority (about $97 \%$ ) of all galaxies more massive than about $1.5 \times 10^{10} M_{\odot}$ in baryons are of late type.

\subsection{Conclusion on testing for the existence of dark matter}

\subsubsection{Test1 and Test2}

Given the failed tests I and II of the dual dwarf galaxy theorem on observational data within the framework of the SMoC, and the unfavorable test of the SMoC using dynamical friction, the following can be concluded:

RESULT: According to both tests I and II above: type A dwarfs $=$ type $\mathrm{B}$ dwarfs. This falsifies the dual dwarf galaxy theorem if the SMoC were true. Thus the SMoC, and more precisely, the existence of cold or warm dark matter particles, appears to be ruled out. The lack of evidence for dynamical friction also implies the cold or warm dark matter framework, and thus the $\mathrm{SMoC}$, to not be an adequate description of galaxies.

\subsubsection{Implication}

By inference the observational evidence suggests the following implication:

IMPLICATION: The ruling-out of the existence of dynamically relevant cold or warm dark matter particles implies the dynamics of galaxies need to be accounted for by an effective nonNewtonian theory. That the hitherto observed late-type (i.e. star-forming) TDGs lie very near to the BTFR defined by the PDGs suggests that the dynamics should be given by a fundamental principle not related to exotic dark matter (Sec. 6).

\section{Robustness of the falsification}

The observational evidence thus points towards dynamical friction not playing a dominant role in galaxy evolution and galaxy-galaxy interactions. Put differently, if anything, the data are easier to understand with dynamical friction being suppressed. There is no convincing observational evidence for dynamical friction at work. This test is not as clear cut though as the tests based on the dual dwarf galaxy theorem, since for example the modelling of the M81 group appears to have stopped once two independent groups reported in conference proceedings that dark-matter halo solutions have been found to fail to account for the observed distribution of matter and the line-of-sight kinematical information. Similarly, the Sgr satellite galaxy problem requires much more attention with Sgr being embedded in its own dark matter halo, because most of the effort has shied away from this critical approach, although in easy reach of numerical work.

The two-fold test using the dual dwarf galaxy theorem is, however, robust and significant, but it is subject to the observational evidence remaining valid. The observed Universe does not provide any evidence for two types of dwarf galaxies to exist which can be differentiated in terms of their dark matter halos. The observed TDGs appear to have just as much dark matter as PDGs.

OVERALL CONCLUSION: Combining the tests on dynamical friction and on the dual dwarf galaxy theorem suggests very strongly that dark matter halos are not present. Dynamically significant exotic dark matter particles do not appear to exist.

Clearly, while some might be convinced, most astronomers and physicists will not be. Reluctance to accept this conclusion is understandable and comes twofold: a well-founded inertia to depart from GR and a sociological inertia given by a wellestablished professional landscape. ${ }^{8}$

\footnotetext{
${ }^{8}$ A part of the research community appears to have fallen into the trap "We know there is dark matter therefore it is not possible that there is none". As an example of the certitude in parts of the community, [170] exemplifies this certainty in the community that dark matter exists by writing "Due to a large number of astrophysical observations ... we know today that dark matter exists" (originally: "Aufgrund einer Vielzahl von astrophysikalischen Beobachtungen ... wissen wir heute, dass Dunkle Materie existiert" ) and "The question is thus not: does dark matter exist? Rather, the issue is to find out: what does it consist of?" (originally: "Die Frage ist also längst nicht mehr: Existiert die Dunkle Materie? Vielmehr gilt es herauszufinden: Woraus besteht sie?"). As another example, a
} 
The "no-dark-matter" deduction has major implications, both for fundamental physics and sociologically. The implications for fundamental physics are highly exciting, since we are, in essence, facing a possible departure from Einstein's GR. At the least we may obtain enhanced knowledge of space-timematter-vacuum physics (see e.g. [171], Sec. 6.3 and 8). Sociologically and politically the implication is that searches for the exotic dark matter particles would be expected to be fruitless. But many resources have been invested in this avenue of research.

Given the gravity of the "no-dark-matter" deduction, it is necessary to make sure that it is held-up by independent evidence. Self-consistency checks are thus required.

SELF-CONSISTENCY: If the above "no-dark-matter" deduction were to be true, then the SMoC should not be a successful description, or in the least, it should not be a unique description of the observational data. That is, how well does the SMoC truly account for the observed extragalactic structures?

While this question has already been answered in Sec. 4.2 (the SMoC has not successfully accounted for the galaxy population and its spatial distribution), in the following a few additional considerations are discussed in order to further illuminate the "no-dark-matter" deduction.

\subsection{Which satellite galaxies are TDGs without dark matter, and which might be PDGs with dark matter?}

That the type A dwarfs and the type B dwarfs are, within the observational uncertainties, apparently indistinguishable in terms of their dynamical and morphological properties, suggests the SMoC to be false. Given the current observational situation, we thus have

type A dwarf = type B dwarf.

This may be due to only type A (i.e. dark-matter-dominated PDGs) existing, with type B dwarfs (i.e. TDGs) disappearing rapidly after their formation. However, the bulk of the evidence does not favor this possibility, because TDGs are observed and it has already been well established, both observationally and by high-resolution numerical work, that TDGs survive for many Gyr (Sec. 3.2). Furthermore, this would not alleviate the problem with dynamical friction discovered above.

An alternative is to assume all dwarf galaxies are TDGs. All dwarf galaxies cannot, however, be TDGs because isolated late-type dwarf galaxies and dwarf-galaxy groups [172], as discussed e.g. in [173], cannot have formed as TDGs.

cosmologist who is a life-long director of a North-German research institution walked out in protest after about one third of the invited colloquium at the director's institute which was being given on this topic in February 2014. Although no-one from the packed audience followed, and the seat was quickly refilled, this incidence does indicate an interesting attempt at exerting peer pressure, especially since it may be employed thereafter tactically when referring to an "impossible speaker". It has also occurred that authors of submitted manuscripts were required, on occasion, by the referee and editor of major journals to drop references to peer-reviewed papers which are SMoC-critical, such that e.g. students reading such manuscripts would not be exposed to such critical studies.
But it may be posible that the majority and perhaps all satellite dwarf galaxies are TDGs. In this case most satellite systems ought to show anisotropies such as flattened rotational populations of dwarf galaxies around their host galaxy because TDGs from in tidal tails. This will be true if interactions between major galaxies are typically less frequent than twice per Hubble time, because one encounter is required to produce the population of TDGs while a second encounter would disperse the satellit system reducing its anisotropy (see Sec. 9, prediction 9.3).

An important consistency check on the conclusions reached in Sec. 4 is therefore given by the spatial distribution of dwarf satellite galaxies around major host galaxies, as discussed in the following.

\subsubsection{Anisotropy of satellite distributions and the origin of galactic satellites}

Observational evidence for a population of satellite galaxies in a correlated distribution would imply these to be most likely TDGs, because no other mechanism is known to generate significantly phase-space-correlated distributions of satellite galaxies. From Sec. 3.2 it follows that populations of TDGs around one host galaxy are likely to be in phase-space correlated structures, as long as the host did not experience more than about one encounter per Hubble time, assuming one major encounter can randomise a previously formed disk of satellite TDGs. A small number of encounters per galaxy is expected to be the case because the vast majority of host galaxies are late-type rotationally supported disks which preclude them to have had many encounters (Sec. 4.2.4). Given eq. 1, we would have a significant consistency argument supporting the "no-dark-matter" deduction if such anisotropically distributed satellite galaxies were to be found.

This argument would be very strong if it were to be found that some satellite population is so anisotropic and correlated in phase space (e.g. by a large number of satellite dwarf galaxies orbiting their host galaxy in one and the same sense in a relatively thin disk-like structure) that the hypothesis that they might be independently accreted dark-matter-dominated (type A) dwarfs can be excluded with very high confidence. This is indeed the case:

\subsubsection{The case with the best data: the Milky Way:}

Considering the positions of the then-known (bright) satellite galaxies and of globular clusters of the MW in the 1970s, Lynden-Bell [174, 175] and Kunkel \& Demers [176] realized their arrangement in a Galactic near-polar band on the sky. The natural interpretation of this structure was it to have been born from the tear-up of a larger Magellanic Cloud. However, with the advent of the SMoC in the 1990s and its prediction that a MW-sized galaxy should have a large number of dark-matter dominated satellite galaxies (the satellite overprediction problem, $[81,82])$ and the observation that the real $\mathrm{dSph}$ satellite galaxies have very large dynamical $M / L$ ratios [90] this interpretation was discarded and attention of the research community was drawn to dealing with the satellite overprediction problem. The significant discrepancy of the SMoCprediction of a spheroidal dark-matter dominated satellite distribution with the observed disk-of-satellite (DoS) distribution, 
taking into account all then-known satellite galaxies, was documented for the first time by Kroupa et al. (2005, [177]). That the high dynamical $M / L$ ratios may be feigned by the satellites being non-virial-equilibrium and largely unbound remnants of satellite galaxies gave support to an interpretation of the dwarfs as ancient TDGs despite their large dynamical $M / L$ ratios $[111,114,115,178,179]$

The recent work of Pawlowski et al. [8, 9, 96] has shown that this vast polar structure (VPOS) around the MW is known today to host virtually all known satellite galaxies (27 in number, Fig. 2, this includes Crater or PSO J174.0675-10.8774) and all the young halo globular clusters (YHGCs) as well as a significant fraction of all known gas and stellar streams. The highly significant existence of the very large $(\approx 500 \mathrm{kpc}$ in diameter) planar or disk-like (thickness $\approx 50 \mathrm{kpc}$ ) distribution of material around the MW $[8,85]$, which we know to be mostly rotating in one sense $[92,180]$, can most naturally, and probably only, be explained as the MW satellite galaxies being old TDGs [93, 106, 178]. Attempts at accounting for this rotationally supported vast structure around the MW in terms of the infall of dark-matter, i.e. primordial or type A dwarf satellite galaxies, have been excluded with extremely high confidence $[9,86,173]$. The infall of a group of or filamentary accretion of dark-matter dominated PDGs are ruled out as well (Sec. 5.1.4).

\subsubsection{The Andromeda system (GPoA):}

Recently, Ibata et al. [6] and Conn et al. [7] verified and quantified the existence of a great plane of Andromeda (GPoA, sometimes also referred to as the vast thin disk of satellites, VTDS) which contains about half of all satellite galaxies of Andromeda. It consists of old gas free dSph satellite galaxies and is very thin $(\approx 14 \mathrm{kpc}$ in thickness $)$ and extended ( $\gtrsim 400 \mathrm{kpc}$ in diameter) and the satellites are moving about Andromeda in bulk. That is, this GPoA structure is rotating (Fig. 3). Given attempts to argue that this structure arises "naturally" or "commonly" in SMoC simulations, [89] have studied such claims. They performed significance tests and they have calculated the likelihood that such a GPoA structure can be obtained from dark-matter sub-halos. They write "We find that $0.04 \%$ of host galaxies display satellite alignments that are at least as extreme as the observations" considering the "extent, thickness and number of members rotating in the same sense". The only viable explanation for the GPoA structure appears to be a remnant of an ancient tidal arm within which satellite dwarf galaxies formed as TDGs (see e.g. [106]).

The GPoA is significant evidence that type A dwarfs cannot be distinguished dynamically nor morphologically from type B dwarfs (i.e. that eq. 1 holds). This is the case because the dSph galaxies in the GPoA appear in every respect to be the same as those apparently dark-matter dominated satellite galaxies that are not in this GPoA.

That the MW VPOS and Andromeda GPoA may be physically related is suggested by both systems having the same sense of rotation with respect to the Local Group coordinate system (Fig. 2 and 3). This may be understandable to be a consequence of both satellite systems being born as TDGs during an encounter between both major galaxies about $11 \mathrm{Gyr}$ ago [181]. Since then the MW and Andromeda would have evolved independently. There is evidence that Andromeda had a more complex past by experiencing further minor encounters with its own satellites. This evidence is discussed by [182], who also summarize our knowledge of the present-day properties of both galaxies. Evidence for a rich history of encounters of Andromeda is studied by Hammer et al. [183] as well. An innovative solution to the VPOS/GPoA correlation problem is suggested by $[105,179]$, where it is shown that a merger in Andromeda about 6 Gyr ago reproducing tidal features around Andromeda would have expelled a tidal tail within which TDGs formed. The remarkable aspect of this model is that without additional parameter adjustments, this tidal tail turns out to have passed the MW such that the MW satellite galaxies which are today arranged in the DoS/VPOS may be the TDGs that formed in this tidal tail. While mergers appear to be rare in reality, some may occur. It will be useful to investigate this scenario in a dynamical model without exotic dark matter particles (Sec. 6). The highly symmetrical structure of the dwarf galaxy arrangement in the Local Group (Pawlowski, Kroupa \& Jerjen 2013, [91]) may be suggestive of the Local Group dwarfs having formed as TDGs. Further observational indications of a TDG-origin of dwarf galaxies nearby the Local Group in terms of the structure of the Perseus I and NGC 3109 association has been discovered [184].

As pointed out by [91], there is evidence that Andromeda has another disk of satellites which is co-planar within the disk of Andromeda. Such a DoS would not be observable around the MW because of the obscuration of it through the MW.

\subsubsection{The correlated satellite systems of NGC1097, NGC5557, NGC4631, NGC4216, NGC5291, M81 and the Tadpole:}

Other galaxies have been found to also have phase-spacecorrelated satellite populations. Examples are the dSph satellite galaxies in the "dog-leg" stream of NGC1097 [185], the linear arrangement of 2-3 Gyr old dSph satellites around the post-interaction remnant NGC5557 [123], the three satellites in the tidal feature of NGC4631 [186], and the arrangement of satellite galaxies around the spiral NGC4216 [187]. The Tadpole (Fig. 4) is another case of a strongly correlated young population of star clusters, star-cluster complexes and low-mass TDGs, as is the vast gas ring with TDGs around NGC5291 [122]. Last not least, [188] discuss the dwarf galaxy population in the M81 group of galaxies, which is a sparse group comparable to the Local Group, and they find evidence that the faint satellite galaxies are distributed anisotropically. They write "In review, in the few instances around nearby major galaxies where we have information, in every case there is evidence that gas poor companions lie in flattened distributions."

This proves that the two major galaxies of the Local Group are no exception. The above discussion also suggests what the dominant origin of satellite galaxies appears to be. All known satellite galaxies of the MW are members of the VPOS such that at least the majority of them would be ancient TDGs. The Andromeda satellite system is richer than that of the MW. About halve of all the satellites of Andromeda are in the GPoA that rotates in one sense. Thus, at least about halve of the Andromeda satellites would be ancient TDGs. Since these do not 
differ in any discernible way from the other satellites, it appears reasonable to assume that most of Andromeda's satellite galaxies are also TDGs.

EMPIRICAL EVIDENCE: At least nine host galaxies have been documented to have satellites which are correlated in phase space. Thus, most dwarf satellite galaxies may be TDGs.

\subsubsection{The mass-metallicity relation of TDGs}

Galaxies are known to follow a mass-metallicity relation which is largely understood as a result of more massive galaxies having larger star-formation rates, lesser galactic winds and increasingly top-heavy galaxy-wide stellar initial mass functions (IMFs; [189-192], see also Sec. 7). An important question is whether TDGs, which form from pre-enriched gas, also follow such a relation. This is particularly relevant for the satellite galaxies of the MW and of Andromeda: if they are TDGs as is suggested by them populating correlated disk-like distributions around their host galaxies, then how can they follow a mass-metallicity relation of PDGs? The essential argument here is that by ruling-out the existence of exotic dark matter above, it transpires that galaxies all follow the same gravitational rules (Sec. 6). The evolution in the mass-metallicity diagramme will thus be indistinguishable, apart from possible physical processes such as ram-pressure stripping which contributes to the truncation of star-formation activity in a lowmass satellite dwarf galaxy, and the tracks will depend on an initial pre-enrichment of the gas in the tidal arm from which the TDGs form.

Fig. 8 shows the theoretical mass-metallicity relations of TDGs in comparison to observed TDGs (upper panel) and to the dSph satellite galaxies of the MW and Andromeda (lower panel). The models are computed with the IGIMF theory (the galaxy-wide star-formation-rate(SFR)-dependent IMF, Theory C in Sec. 7.5). The models are modifications of standard leakybox models [193] in which the hypothesis of an invariant IMF is relaxed [196]. The top panel shows gas abundances in comparison to observed TDGs. The lower panel shows stellar abundances derived from the same models in comparison with MW satellites. The different curves have different initial metallicities corresponding to initial pre-enrichment. Thus, the MW and Andromeda dSph satellites formed as TDGs about $11 \mathrm{Gyr}$ ago when the gas in the outer region of the pre-encounter galaxies was not much pre-enriched, while the present-day TDGs (upper panel) formed from pre-enriched material, as is to be expected.

Conclusion: Using standard chemical evolution models (with the IGIMF) dSph satellites and TDGs naturally fit the massmetallicity relation when one takes into account that they form at different epochs from pre-enriched gas.

The observed mass-metallicity relation of dwarf satellite galaxies is thus consistent with them being TDGs.

\subsubsection{SMoC-internal conflicts and inconsistencies}

It is important to assess whether dwarf galaxy satellite structures similar to the observed VPOS and GPoA can result within the SMoC in terms of the distribution of dark-matter sub-halos, because the dSph and UFD satellite galaxies within the VPOS
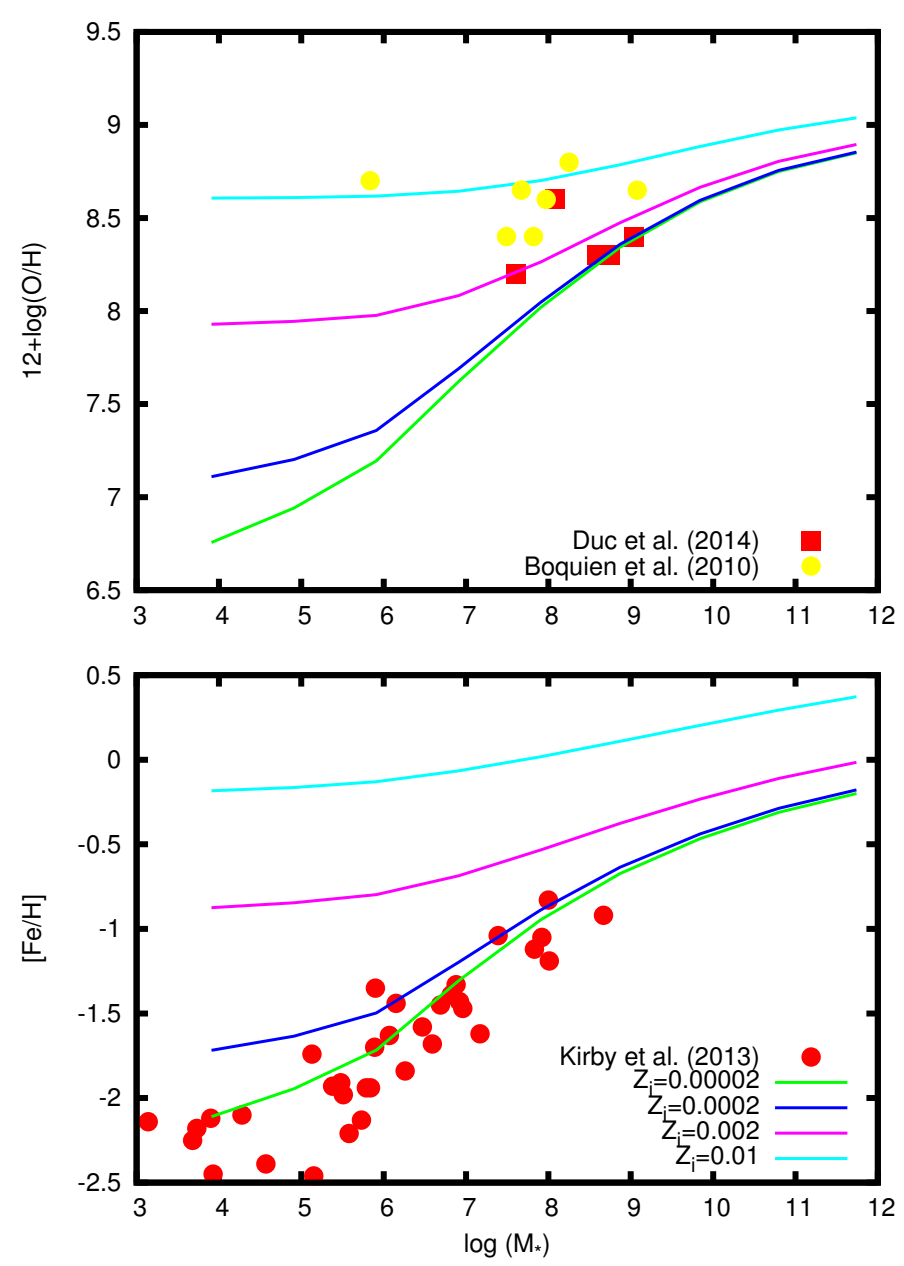

Fig. 8. The mass-metallicity relation of TDGs. The curves are one-zone leaky-box chemical evolution models (compare with [193]) starting the chemical evolution at an initial metallicity, $Z_{i}$, as given in the key. The galaxy-wide stellar IMF is assumed to be the star-formation-rate-dependent IGIMF (Sec. 7). The outflow rate is chosen to be larger in low-mass galaxies, smaller in more-massive ones, in compliance with the results obtained by [191]. The upper panel shows the oxygen abundance in the gas phase, as compared with observations of TDGs (from [108, 194]). The lower panel shows the stellar [Fe/H] abundances predicted by the same models in comparison with observations of MW and M31 satellites summarized in [195]. The lower panel shows thus that the MW and Andromeda satellite galaxies are consistent with the laws of star formation (Sec. 7), if the pre-enrichment $Z_{i}$ is very low. The same models able to fit the satellites of the MW and M31, are able to fit the oxygen content of younger TDGs (upper panel), with different degrees of pre-enrichment (from very low to moderately-high). 
and GPoA appear to be the most dark-matter dominated galaxies known to exist [90]. If dark-matter-made VPOS or GPoAlike structures cannot arise in the SMoC then an inconsistency between theory and data arises and the $\mathrm{SMoC}$ would need to be discarded in agreement with the conclusion reached in Sec. 4. The inference on the dark-matter content of the satellite galaxies is valid if Einsteinian/Newtonian dynamics is applied in analyzing the structural and kinematical data of the stars orbiting within the satellite galaxies. If the VPOS and GPoA structures cannot arise in the $\mathrm{SMoC}$, then the observed high dynamical $M / L$ ratios of the MW and Andromeda satellite galaxies would hint at effective non-Einsteinian/non-Newtonian dynamics (see also Sec. 6).

How mutually consistent are the various attempts at accounting for the VPOS and GPoA structures, that is, are inconsistencies and perhaps even mutually logically exclusive results evident in applying the SMoC to the VPOS and the GPoA? Incompatible results, if they exist, point towards the underlying theory being wrong.

Consulting the available published research it is indeed evident that consistency issues do arise. In attempting to reproduce VPOS- and GPoA-like structures with dark-matter subhalos, various teams may focus on one or a few aspects thereby possibly neglecting the wider context or even similar attempts by other teams who may have come up with solutions that are inconsistent with the new proposal:

How should the community judge the scientific situation if a team $\mathrm{T} 1$ proposes a $\mathrm{SMoC}$ solution A to some phenomenon, and if team T2 proposes a SMoC solution B to the same phenomenon, but the solutions A and B contradict each other, which, however, is neither discussed by $\mathrm{T} 1$ nor by $\mathrm{T} 2$ ?

Two important contributions illustrate this situation: [197] find that in SMoC simulations the recent infall (at redshift $z<$ 1) of a group of type A dwarfs may account for an anisotropically distributed population of satellite galaxies at the present $(z=0)$. This confirms [198] who write "Although the satellites often form groups, they are loosely bound within them and do not interact with each other. The infall of a large group could however explain the observed peculiar distribution of the LG satellites, but only if it occurred recently.” But, [199] obtain the result that in order to explain the observed lack of gas in the MW satellites, they must have fallen in early at $z=3-10$. Both are mutually exclusive but claim consistency with the data and the model. This discrepancy lies in [197] not accounting for the MW satellites being void of gas, while [199] do not account for the VPOS. But the MW satellites are both, gas poor and in the VPOS:

MUTUALLY INCONSISTENT RESUlTS: For the VPOS to be accounted for with PDGs the MW satellites must have fallen in at a high redshift and at the same time at low redshift. Given the observational data, this logically incompatible result invalidates the underlying hypothesis (that the SMoC is valid), unless one of the studies needs to be revised.

The Durham team around Carlos Frenk has been addressing the VPOS challenge for the SMoC with important results. Of $400 \mathrm{MW}$-scale dark matter halos that form in their cosmological simulations, the occurrence of the DoS/VPOS around the MW becomes negligibly likely if more than 8 satellite galaxies have orbital angular momenta aligned to within 45 deg (fig. 9 in Libeskind et al. 2009, [200]). [197] confirm this result. As shown by [92], taking into account the additional cuts that are required to end up with the MW-like satellite system in the SMoC simulation of the Durham team, the number of MW satellites with orbital angular momenta as closely clustered as is observed becomes negligible with a likelihood less than about $0.5 \%$ (see also a detailed discussion of this in [62]). Thus, according to the Durham team neither the VPOS nor the GPoA can be accounted for with primordial, dark-matter dominated dwarf galaxies. Since the publication of the Durham analysis, further SMoC teams working on this issue have not addressed this conclusion, while postulating their own solutions (e.g. [201-203]). Libeskind (2011, [204]) write "While the planarity of $M W$ satellites is no longer deemed a threat to the standard model, its origin has evaded a definitive understanding.", and study anisotropic infall of PDGs into the MW halo to better understand its satellite distribution but do not apply their results for a direct statistical assessment of whether the VPOS may be explainable this way.

For example, in attempting to explain the GPoA around Andromeda in terms of PDGs with dark matter, [202] write in their abstract that "we find that planes with an rms lower than the VTPD are common in Millennium II" but in the body of the paper the probability of this occurring is stated to be $2 \%$, which is not common. As pointed out by [89] the analysis by [202] is flawed by it not taking into account the appropriate criteria $^{9}$, and the true likelihood of the occurrence of systems comparable to the GPoA is negligible in the SMoC $(0.04 \%)$. The proposition by [203] that GPoA- and VPOS-like structures can be understood naturally within the $\mathrm{SMoC}$ as arising from a few thin filaments connecting to the host galaxy along which the $\gtrsim 20$ dark-matter dominated satellites accrete has, at the time of writing of this text, not been accepted for publication. But the manuscript is already being cited in the community as having shown that GPoA- and VPOS-like structures do arise naturally in the SMoC (e.g. [202,205]). Pawlowski et al. (2014, [9]) analyse and rule-out the proposition by [203] in detail.

Metz et al. [85] have developed statistics tools from the mathematical literature to assess the degree of clustering of data

\footnotetext{
${ }^{9}$ Of the three criteria that define a disk-like distribution of satellite galaxies, [202] have adopted two at a time yielding a probability of occurrence of such a structure within the $\mathrm{SMoC}$ simulation data of $2 \%$ which [202] describe as being common. [89] confirm the [202] likelihoods and show that the three criteria that are in truth required to define GPoA-like structures lead to a combined likelihood of occurrence of GPoA structures of $0.04 \%$, consistent with their own independent calculation based on the cosmological simulation data and with those of [62]. That is, obtaining VPOS or GPoA-like structures does not seem to be possible in the SMoC if these are to consist of PDGs. [89] give the following example which visualises why using the correct selection criteria is essential for inferring the workings of nature (cited from their footnote 8): "To make the issue perfectly clear, consider measuring the incidence of animals that have stripes and paws and are nocturnal. Clearly, selecting only two of these three properties will yield a larger (and incorrect) sample of such animals, giving a falsely optimistic measurement of how common they are."
} 
distributed on the sphere in terms of strength and shape parameters. These tools have been tested carefully and proven to be ideal for robustly determining the significance of satellite anisotropies given their three-dimensional spatial information $[8,85]$ :

The scientific community is highly encouraged and invited to compare their own criteria for quantifying the statistical likelihood of satellite anisotropy in cosmological models to the robust method of [85] (see also the above discussion and $[9,89]$ ).

Some recent work on the distribution of satellite galaxies continues to only concentrate on their radial distribution. For example, [87] state "The accurate prediction for the spatial distribution of satellites is intriguing given the fact that we do not explicitly model satellite-specific processes after infall, ...” but do not mention the significant anisotropy of the satellite galaxy distribution now established for the MW and Andromeda.

At the same time, [88] stress that "current semi-analytic simulations, where satellite profiles always parallel those of the dark matter". This recent contribution does not address the question posed originally by [177] why the satellites of the MW and Andromeda are in thin planes while the host dark matter halos are spheroidally distributed. Furthermore, that the model satellite galaxies violate the observed lack of a correlation between the satellite masses and their luminosities has not found an explanation in the SMoC framework, where such a correlation needs to be present [62].

It has been appreciated since a long time [174-176] that the Magellanic Stream and the orbits of the Large and Small Magellanic Clouds (LMC and SMC, respectively) define a plane within which also lie globular clusters and dwarf spheroidal satellite galaxies. Today this is known as the DoS or VPOS (Sec. 5.1.1). The most recent work on the orbital evolution of the Large and Small Magellanic Clouds by Besla et al. (2012, [206]) studies, for the very first time, the two galaxies selfconsistently within the SMoC. Therewith this is a landmark study which has advanced knowledge of the Magellanic system appreciably. The LMC and SMC have, respectively, DM halo masses of about $2 \times 10^{11} M_{\odot}$ and $2 \times 10^{10} M_{\odot}$ with radii of $R_{200}=117 \mathrm{kpc}$ and $R_{200}=57 \mathrm{kpc}$ where the enclosed average density is 200 times the critical density of the universe. For the MW the authors use a DM halo mass of $1.5 \times 10^{12} M_{\odot}$ with $R_{200}=220 \mathrm{kpc}$. This halo is treated as a rigid potential, while the LMC and SMC halos are live. Thus, dynamical friction occurs between the LMC and the SMC but is neglected for both as they orbit through the MW DM halo. The results are useful in interpreting whether the two galaxies are on a first-time passage around the $\mathrm{MW}$, and the authors find that an encounter between the LMC and the SMC generated tidal tails which are observed as the Magellanic Stream today. The authors stress that they are not intending to reproduce the exact properties of the stream, but the existing models do account for overall properties of it. Future work will have to be done to assess how neglecting dynamical friction of the LMC and the SMC on the MW DM halo affects the conclusions, and if solutions may be found at all. Also, a discussion is lacking entirely as to the coincidence of the plane of the first-time incoming orbit of the LMC and SMC being oriented within the VPOS such that the resulting Magellanic Stream also lies within the VPOS although it is created in a pre-infall encounter between the LMC and the SMC. Thus, the fine-tuning between the VPOS and the LMC-SMC binary orbit with semi-major axis near $100 \mathrm{kpc}$, this orbit and the hyperbolic orbit of the $\mathrm{LMC} / \mathrm{SMC}$ barycentre around the MW, and the encounter geometry between the LMC and SMC such that the Magellanic Stream is produced which lies within the VPOS although it is generated as tidal tails well before the LMC/SMC binary has crossed the MW $R_{200}$ radius, remains to be discussed. This is thus a situation reminiscent of the M81 case of Sec. 4.2.3. As is evident, these models raise important questions and they are therefore essential and valuable for gaining a deeper understanding of processes that may be playing a role in establishing the properties of the Local Group.

Leaving the problem of the VPOS and of the GPoA and other anisotropic satellite systems, it is not uncommon indeed to perform simulations within the framework of the SMoC of the motions of satellite galaxies by ignoring dynamical friction. Thus, [207] study the formation of ultra-compact dwarf (UCD) galaxies from nuclei of stripped dE galaxies. For computational ease the expansive dark matter halos of the dE satellites are not treated, but dynamical friction of such sub-halos on the massive dark matter halo of the host galaxy would lead to the merging within one or two crossing times thus possibly eliminating any viable solution to the problem altogether. For example, a dE galaxy with a baryonic mass of $M=10^{8} M_{\odot}$ would have a dark matter halo of $M_{\mathrm{DM}} \approx 10^{10.5} M_{\odot}$ while a $\mathrm{dE}$ with with $M=10^{9} M_{\odot}$ would have $M_{\mathrm{DM}} \approx 10^{11.2} M_{\odot}$ $[70,124]$. By footnote 7 a short dynamical friction time scale obtains assuming the host galaxy to have a dark matter halo $M_{\mathrm{DM}} \approx 10^{12} M_{\odot}$. Since dynamical friction is not taken into account, it remains unknown if any viable solution to the problem of UCD formation exists, but the published work states "we consider tidal stripping to be a likely origin of UCDs".

Concluding, it is thus apparent that while important and useful work has been done within the framework of the SMoC, typically such work had to neglect important aspects or has not taken into account the conclusions reached by others or has even been contradictory, time and again. The results thus arrived at within this framework are not convincing, although being important for aiding to assess the possible validity of the SMoC.

\subsubsection{Cold filamentary infall}

In order to account for the gas accretion onto galaxies and their star-formation histories, [208, 209] proposed that gas accretes into the host dark matter halos in long-lived gaseous filaments that are thinner than the virial radii of the host dark matter halos. This fuels the formation of major galaxies. [210] demonstrate with cosmological simulations that such very thin cold gaseous filaments arise within a warm dark matter cosmological model and that they feed MW-type galaxies mostly before a redshift $z=2$ after which the streams subside. Such filaments reside in the much thicker dark matter web.

One attempt at reconciliating the existence of the VPOS and of the GPoA with dark-matter (type A) PDGs is to postulate that these structures are a result of infall from cosmological filaments. The incompatibility of late versus early infall noted in Sec. 5.1.3 persists but is not usually addressed.

[92] and [91] analyse in much detail the claims that the 
VPOS of the MW and the GPoA of Andromeda may be a result of filamentary infall and demonstrate that these claims can be ruled out. Basically, the cosmological simulations show the dark matter filaments or sheets to be too thick to account for the thin VPOS (thickness about $50 \mathrm{kpc}$, diameter about $500 \mathrm{kpc}$ ) and the even thinner GPoA (thickness about $14 \mathrm{kpc}$, diameter about $400 \mathrm{kpc}$ ).

Perhaps the last hope would be to assume that the VPOS and GPoA would stem from accretion of dwarf galaxies along cold filaments [203]. This proposition has at least two problems: Firstly, it would require the cold filaments of baryons to be populated by dark matter sub-halos which would then fall into the hosting halo like beads on a string with thickness less than $50 \mathrm{kpc}$ (Fig. 9). Such beads of dark matter subhalos, each with a virial radius of about $100 \mathrm{kpc}$ given that they would have to be hosting pre-infall dwarf galaxies that are to become MW or Andromeda dSph and UFD satellites (for their putative dark matter halo masses see [70,124] and for their radii see [138]), have never been shown to arise in any self-consistent structure formation computation, and it is difficult to see how such highly-focussed infall of dark matter halos could arise. The baryonic-matter stream cannot influence the orbital path of dark matter sub-halos, given that the baryonic mass component is small compared to the dark matter mass component. That is, it is difficult to see a physical mechanism by which the cold gaseous filaments align at least about 20 PDGs which later, after infall, become all of the known satellite galaxies in the VPOS and GPoA. The second problem is that the streams are radial, and infall would have to have occurred at a redshift larger than $z=2$ [199]. It is unclear how disks of satellites are to arise at a redshift of $z=0$ since a given sub-halo would fall-into a host halo and would launch on a Rosetta-type orbit with changing orbital orientation in the typical triaxial host dark matter halo. Each satellite would end up on a different orbital plane, subject to dynamical friction.

COLD FILAMENTS: Infall from cold filaments of dark-matter dominated dwarf galaxies can be ruled out as an explanation for the existence of the VPOS and GPoA.

\subsection{Falsification and the theory confidence graph}

In principle a single robust observation which significantly contradicts a predicted phenomenon suffices to falsify the underlying theory. In practice it is important to understand if the prediction, in the face of failure, may not be amendable by including previously not accounted-for physical processes.

Thus, for example, the "satellite over-prediction problem" (i.e. the "missing satellite problem", [81, 82]) has not been taken to rule out the SMoC. Instead, many research teams attempted to reconcile the much larger number of predicted PDG satellites in MW-type host halos with baryonic-physical processes, such as suppression of the formation of stars due to reionisation, or suppression of satellite galaxy formation through destructive feedback processes. It has indeed become possible to account for the small number of observed MW and Andromeda satellites in this way, but with the caveat that many hundreds of even fainter satellite galaxies distributed spheroidally around the MW or Andromeda should be found once the surveys become more sensitive than the SDSS. Thus, [211] provide "a convincing solution to the "missing satellite" prob-

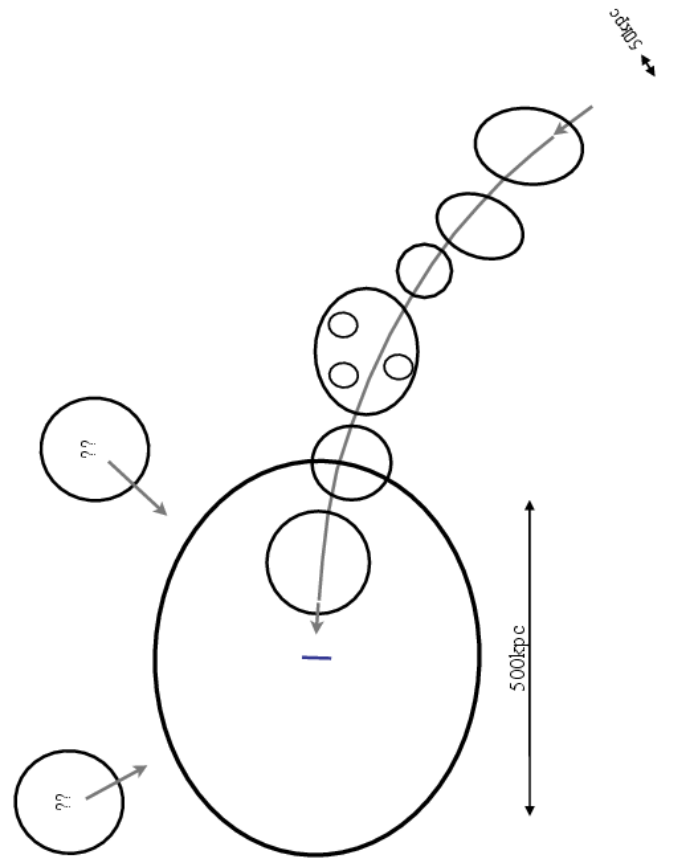

Fig. 9. Cartoon of how the infall of a sequence of about 20 or more PDGs would need to have occurred for the resulting distribution to become comparable to the VPOS (Fig. 2) or GPoA (Fig. 3). The host galaxy is shown as the edge-on solid disk and its hosting dark matter halo is the near-circular black outline with a diameter of about $500 \mathrm{kpc}$. The grey line indicates a cold filament with thickness no larger than $50 \mathrm{kpc}$ along which a long sequence of dark-matter sub-halos need to fall in. The diameters of these are between 100 and $200 \mathrm{kpc}$ but their centres need to follow the cold filament. More than one or two dark matter sub haloes cannot accrete from elsewhere onto the host halo, in order to preserve the VPOS of the MW. Note that some of the sub-halos may have their own sub-sub-halos. 
lem.", but do not address the DoS/VPOS problem. Similarly. [99] state "that there are likely between $\sim 300$ and $\sim 600$ satellites brighter than Boo II within the Milky Way virial radius". This gives new predictions which can be falsified with deeper more sensitive surveys.

However, if a theory has a history of failed predictions each of which need individual attention in searching for possible solutions within the theory, the scientific community has a right to loose confidence in the theory, independently of the availability of a single cataclysmic failure (such as the violation of the dual dwarf galaxy theorem):

In fact, if a single cataclysmic failure has been shown to exist, then this failure, if correct, implies the theory to not be valid. In this case this theory should have a history of failures since it does not describe reality. A history of failures would be equivalent to a history of loss of confidence.

One possibility to assess the confidence in a theory is through the confidence theory graph [5]. To construct this graph one begins with a confidence of one hundred $\%$ at the point in time when the theory is mathematically formulated whereby parameter values are taken to be part of the definition of the model. Each time a prediction is found to be inconsistent with observational data a step downwards is taken in the logarithm of the confidence that the theory is correct. The step may, in the simplest application, be equal in every reported failure, or it may depend on the gravity of the failure. This would, however, require a quantification of the loss of confidence which is difficult.

One example are the many tests of the SMoPP which have, to this date, not found significant failures. The SMoPP thus remains near a confidence of $100 \%$. The fact that neutrinos have masses requires an extension of the SMoPP and can be considered a step downwards in the confidence graph and the question of the origin of inertial mass has been clarified though the discovery of the predicted Higgs boson.

In comparison, the $\mathrm{SMoC}$ has been found to fail massively in its expansion behavior. This is remedied by introducing the novel but not understood physical property of dark energy. This "failure" is thus considered to be the discovery of major new physics, in particular since it is possible to fix the failure in the mathematical description of the theory through introduction of Einstein's constant. One could, in this case, allow one step upwards in the confidence graph, but only if the new physics can be verified independently (e.g., if the postulated existence of dark matter were to be verified by a direct detection). Nevertheless, if a theory acquires a long history of failed predictions, each of which need the addition of additional hypotheses without independent verification or which cannot be convincingly remedied (e.g. the angular-momentum or cusp/core problems have no remedies to date), the theory can be seen as having lost in confidence.

In [5] a list of at least 22 failures (including the Bullet cluster) of SMoC predictions is provided and the theory confidence graph is introduced to graphically document the long history of failed predictions (termed "problems" in the literature). If each confidence-loss step would correspond to a loss of confidence by $50 \%$, the history of failures of the SMoC would amount to a cumulative loss of confidence to a level of $0.5^{22}=2.4 \times 10^{-7}$.
That is, by the year 2012 the observational data have reduced the confidence that the $\mathrm{SMoC}$ represents reality to a likelihood of $2.4 \times 10^{-5} \%$.

Significant failures in the list of [5], for which solutions within the SMoC are not evident, are the high fraction of galaxies with baryonic masses larger than $1.5 \times 10^{10} M_{\odot}$ that are late-type star-forming galaxies ( $\gtrsim 90 \%$ ) rather than early-types ( [152], see also fig. 4.14 in [153]), and the dominant fraction of galaxies without classical bulges (about $60 \%$, [149]). [150] further suggest that the very large fraction (94\%) of disk galaxies have pseudo bulges which appear to be largely ancient but may be rejuvenated during galaxy interactions.

The core/cusp, the angular-momentum and the satellite over prediction problems are still qualitatively conceived to be due to not understood baryonic processes. Energetic feedback, which blows out gas for it to cool and condense back to form a disk galaxy, and the expulsion of which may form the cores of dark matter halos inferred from observations while dark-matter only simulations yield cusps, have been suggested as the remedy. [212] studied this problem using simulations and analytical approaches and conclude "We find that no star formation effect can resolve the problems of CDM cusps." Apart from the typically wrong application of a fully sampled stellar initial mass function (IMF) which would contain unphysical fractions of massive stars (see Sec. 7), observations have shown that gas-outflows that might damage the inner parts of starbursting dwarf galaxies are not evident, despite the injection of $\approx 10^{56}$ erg into the inter-stellar medium (ISM) in the last $\approx 500 \mathrm{Myr}$ [213]. That even repeated outflows from the central regions of simulated galaxies by supernova-driven winds and black hole quasar feedback cannot produce dark-matter cores from cusps is verified by the most recent and novel simulations of galaxy formation using the AREOP5 algorithm by [164]. Based on observational and theoretical work it thus follows:

FEEDBACK: These problems do not have a solution in baryonic physics, contrary to the claims often made in the community even at present times.

The observed Karachentsev-Keenan under-density of matter around the Sun on the scale of 50-400 Mpc appears to be completely in discord with the fundamental hypothesis that the universe is homogeneous. The highly significant under-density of baryonic and dark matter by a factor of three to four within the local volume with radius of $50 \mathrm{Mpc}$ has been noted in 2012 by Karachentsev [68]. A highly significant under-density of luminous and dark matter by a factor of nearly two within $300 \mathrm{Mpc}$ is reported in 2013 by Keenan et al. [69] based on measurements of the photometric K-band luminosity density as a function of redshift (see also [214]). Both, the Karachentsev and the Keenan findings are in significant tension with the SMoC. Either we live in a hole, or there is an unexplained issue with redshift measurements. According to augmentary hypothesis 1 (inflation, Sec. 2) the Universe cannot have fluctuations larger than about $10 \%$ in the density of matter on scales $>100 \mathrm{Mpc}$ (see also fig. 12 in [69] and [215]). The Karachentsev-Keenan under density is depicted in Fig. 1.

An interesting point raised by Keenan et al. [69] is that the observed under density is just the right amount of local inhomogeneity required to annulate the observational evidence for 
dark energy by SNIa. How observed cosmological quantities are affected in an at present inhomogeneous universe over the assumption of a homogeneous SMoC is discussed in terms of timescape cosmology by Wiltshire [215].

In conclusion, while individual solutions to the problems have been suggested in the form of additional hypotheses, most are not generally accepted. The falsification of the SMoC by the tests above is consistent with the (poor) performance of the $\mathrm{SMoC}$ in accounting for the observed Universe.

\section{Scale-Invariant Dynamics (SID)}

Stepping back and reconsidering the problem: galactic dynamics, galaxy evolution and galaxy formation does not seem to be understood within the context of dark matter halos made of exotic particles. With strong if not un-refutable observational evidence against the existence of exotic dark matter, the observed rotation curves of late-type galaxies and the supervirial velocity dispersion in galaxy clusters need to be explained. Note that the evidence by weak gravitational lensing for dark matter in galaxy clusters stems from applying Einstein's GR to calculate, from the weak lensing signal, the amount of matter within the lens. But if this theory is challenged we cannot use it to argue for the presence of dark matter, a trap the carefree researcher can readily fall into.

\subsection{The dynamics of galaxies}

The rules underlying galactic dynamics turn out to be extraordinarily simple, as noted by Milgrom at the Weizmann Institute in Rehovot, Israel ( [216], see also [217]):

Scale the space-time vector by a number $\lambda$,

$(\vec{r}, t) \longrightarrow \lambda(\vec{r}, t)$,

where $\vec{r}=(x, y, z)$ are Cartesian coordinates and $r^{2}=x^{2}+$ $y^{2}+z^{2}$. Under this transformation the kinematical acceleration scales as

$a \equiv \frac{d^{2} r}{d t^{2}} \longrightarrow \lambda^{-1} a$.

The Newtonian gravitational acceleration, $g_{\mathrm{N}}=G M / r^{2}$, however, scales as

$g_{\mathrm{N}} \longrightarrow \lambda^{-2} g_{\mathrm{N}}$.

Here, $G$ is Newton's gravitational constant, $M$ is the relevant gravitating baryonic mass and $r$ the distance to $M$ where $g_{\mathrm{N}}$ is evaluated. One can force the gravitational acceleration to scale invariantly to the kinematical acceleration by redefining it as

$g=\left(a_{0} g_{\mathrm{N}}\right)^{\frac{1}{2}}$,

where $a_{0}$ is a constant that needs to be introduced on dimensionality grounds. Now $g \longrightarrow \lambda^{-1} g$ under eq. 2 . We refer to $a_{0}$ as Milgrom's constant. Eq. 5 thus arises from scaleinvariance of the equations of motions in a gravitational field, and is referred to as scale-invariant dynamics (SID) $)^{10}$.

${ }^{10}$ This had originally in 1983 been called the "deep-MOND regime" by Milgrom [10].
Because the centrifugal acceleration equals the centripetal acceleration for a circular orbit with speed, $v_{c}$, about $M$,

$g=\frac{\sqrt{G M a_{0}}}{r}=\frac{v_{c}^{2}}{r}$.

Thus

$v_{c}=\left(G M a_{0}\right)^{\frac{1}{4}}$,

which is exactly the BTFR (Fig. 5). Thus, if SID were the correct description of dynamics in the ultra-weak-field limit $\left(g<a_{0}\right)$, then all rotationally-supported late-type galaxies must lie on the BTFR (exceptions may occur due to the external field effect, see below). The observed flat rotation-curve data are consistent with eq. 7 for $g<a_{0}$, which is the validity of SID. For $g>a_{0}$ the system approaches the Newtonian regime.

Since the Newtonian circular velocity around $M$ is the circular velocity an observer would see in the absence of dark matter, $v_{c, b}^{2}=G M / r$, it follows by using eq. 7 that

$\left(\frac{v_{c}}{v_{c, b}}\right)^{2}=\left(\frac{a_{0}}{g_{\mathrm{N}}}\right)^{\frac{1}{2}}$.

This is exactly the Sanders-McGaugh mass-discrepancy-acceleration (MDA) correlation which late-type galaxies are seen to obey (Fig. 6).

Here it is important to re-iterate what has been found above: SID: By adopting a simple classical space-time scaling symmetry and forcing the gravitational acceleration to scale as the kinematical one, a law emerges which relates the scaled circular velocity to the Keplerian circular velocity. While as such this law is merely a hypothetical mathematical result, the observational data show it to be excellently fulfilled by nature for describing the observed gravitational law in galaxies. This is shown in Fig. 6 and 7.

Sanders (1990, [54]) pointed out for the first time that disk galaxies obey the MDA relation (eq. 8) and that exotic dark matter halos would not be able to account for it. The MDA relation was verified with many more data by McGaugh (2004, [165]). To construct this law, the observed circular velocity, $v_{c}$, is compared to the circular velocity the star or gas cloud should have in the absence of dark matter, $v_{c, b}$, and their ratio is plotted as a function of the Newtonian acceleration, $g_{\mathrm{N}}$, required for the star or gas cloud to remain on a circular orbit given the baryonic mass of the galaxy. Scarpa (2006, [58]) studied the correlation between the velocity dispersion and luminosity of pressure-supported systems (globular clusters, elliptical galaxies, groups of galaxies and clusters of galaxies). In his fig. 12 impressive agreement between SID and the observations over at least 6 orders of magnitude in acceleration is evident.

Milgrom's constant, $a_{0}$, which is introduced with eq. 5 has the value of about $a_{0}=3.8 \mathrm{pc} / \mathrm{Myr}^{2}$ (e.g. [11,217]). It is unknown at present whether $a_{0}$ is a constant in space and time, but the measurements of it are consistent with it being a true constant of nature. This constant has possible deep associations. A de Sitter universe with a cosmological constant $\Lambda \approx$ $10^{-19} \mathrm{pc}^{-2}$ is characterized by a radius $R_{\Lambda}=1 / \sqrt{\Lambda / 3} \approx$ 
$5 \times 10^{9}$ pc which is about the radius of the observable Universe. Thus $a_{0} \approx\left(c^{2} / 2 \pi\right) / R_{\Lambda}$, which could point to a deeper relation between the two (see [218], footnote 9 in [11] and Sec. 6.3)." Also, constructing the value of an acceleration from cosmological and vacuum constants, we note that

$a_{0}=c H_{0} /(2 \pi)$,

where $H_{0}$ is Hubble's constant such that the observed MDA correlation data are reproduced by eq. 8 (Fig. 6) and $c$ is the speed of light in vacuum. This also suggests a possible relation of SID and of Milgrom's constant to cosmology. Studying the BTFR at high redshifts may provide constraints on a possible variation of Milgrom's constant $a_{0}$ with cosmic time (e.g. [219]). A suggestion that $a_{0}$ may depend on cosmic time within the framework of pure-metric non-Einsteinian gravity models [51] may provide a possible test of such field equations.

The physical origin for the departure of gravitation from Newton's empirical law when $g_{\mathrm{N}}<a_{0}$ is not understood today, but [218] suggests this to be related to quantum-mechanical processes in the vacuum (see also Sec. 8), and [47] has shown a relativistic generalization of Einstein's field equation to provide this departure (for more approaches see Footnote. 4).

SID, as defined above, implies flat rotation curves (eq. 7) and is thus mathematically fully equivalent to the baryonic point mass $M$ sourcing an isothermal dark matter halo about it in Einsteinian/Newtonian dynamics. Because this halo is not made of exotic dark matter particles, but is instead a mathematical property of SID, it is commonly referred to as a phantom dark matter halo.

The phantom dark matter halo has an acceleration at radial distance $r$ of

$g=\frac{v_{c}^{2}}{r}=\frac{\left(G M a_{0}\right)^{\frac{1}{2}}}{r}$.

The Newtonian observer would interpret this acceleration to be due to a (phantom) dark mass, $M_{\mathrm{pDM}}$, within the radius $r$, $g=G\left(M+M_{\mathrm{pDM}}(<r)\right) / r^{2}$, whereby $M \ll M_{\mathrm{pDM}}$ for large $r$ where $g<a_{0}$. It thus follows [156]

$M_{\mathrm{pDM}}(<r)=\sqrt{\frac{M a_{0}}{G}} r$.

The gravitating but not inertial mass of such a phantom dark matter halo can be calculated given that the halo is truncated due to the ambient matter density. The phantom dark matter halo mass as a function of the baryonic mass is given by eq. 5 in [220].

Eq. 10 can be written as

$g=\left(G \pi \Sigma_{M} a_{0}\right)^{\frac{1}{2}}$,

where $\Sigma_{M}=M /\left(\pi r^{2}\right)$ is the baryonic surface density of the disk galaxy in its disk plane evaluated at large $r$ (see also sec. 4.3.2 in [11]). [20] discusses this in more detail and shows that this prediction of SID is verified by observational data. It is unclear how the correlation, $g \propto \Sigma_{M}^{1 / 2}$, can emerge in darkmatter models within the framework of the SMoC.
IMPORTANT: SID implies galaxies to have flat rotation curves, to lie on the BTFR, to obey the MDA correlation and $g \propto$ $\Sigma_{M}^{1 / 2}$

If the galaxy of baryonic mass $M$ is immersed in an external gravitational field with acceleration $a_{e}<a_{0}$, which is constant across the galaxy, then the phantom dark matter halo is effectively truncated at the galactocentric radial distance $r_{t}$,

$r_{t}=\frac{\sqrt{G M a_{0}}}{a_{e}}$,

such that the mass of the phantom dark matter halo is reduced (eq. 11). The depth of the equivalent Newtonian (phantom dark matter halo) potential sourced by this truncated isothermal phantom dark matter mass is thus reduced. Since SID is a non-linear dynamics theory ${ }^{11}$, the influence of $a_{e}$ at all radii plays a role and the gradient of the potential changes and as a consequence the density of the phantom dark matter halo is smaller within $r$ as well. Lüghausen et al. [221] have quantified this in detail. A star orbiting the galaxy of baryonic mass $M$ beyond $r_{t}$ will be accelerated in the external field, while if its orbital radius is within $r_{t}$ it will be accelerated by the galaxy. When $a_{e}>a_{0}$ then the galaxy appears as a naked baryonic system with a Keplerian rotation curve.

A system of baryonic mass $M$, when exposed to an external field, will be spatially more extended than the same isolated system. This comes from the virial theorem according to which a system within a potential must expand if the potential is reduced. A more thorough discussion of this important SID effect can be found in sec. 3.1 of [222], and Sec. 9.7 contains a prediction due to the external field effect.

This external-field effect (EFE) is thus an important prediction from SID. Self-consistent simulations by $\mathrm{Wu} \&$ Kroupa [223] demonstrate the contraction of a system propagating from the Newtonian regime (strong $a_{e}$ ) to the SID regime (weak $a_{e}$ ). Further details on SID and its implication for dwarf galaxies are available in [224] and in [156,221]. Given the EFE, local (i.e. terrestrial) tests of MOND become impossible [225].

Note that the EFE is an unavoidable phenomenon in SID. But is could be different than the simple discussion above suggests: [226] points out that there are two interpretations of SID (or, more precisely of the full classical formulation of Sec. 6.3). The one interpretation is that SID is classical but a non-Newtonian gravitation theory. Another interpretation is for SID to be a theory in which inertial mass does not equal gravitational mass in the SID regime. This latter interpretation leads to SID being non-local in time. This implies that the system's state depends on its full trajectory in phase space. Milgrom writes "A subsystem, such as a globular cluster or a dwarf galaxy, moving in the field of a mother galaxy, or a galaxy in a cluster, may be subject to an EFE that depends on the accelerations all along its orbit, not only on the instantaneous value." It is possible to construct SID theories which have "practically no EFE". "Practically no EFE" here means that in theory there is a small unavoidable effect, but in practice it can be neglected.

\footnotetext{
${ }^{1}$ Note that GR is also non-linear while Newtonian dynamics is linear. Newtonian dynamics can thus be viewed as a linearized approximation of an otherwise non-linear space-time-matter coupling.
} 
Is the EFE evident in observational data?

\subsection{Testing the external field effect (EFE)}

Detailed and self-consistent calculations are required to test this important prediction of SID. But it is possible to already study available data for evidence against or for this prediction.

\subsubsection{Evidence for the $\mathrm{EFE}$}

The satellite galaxies of Andromeda are known to be systematically larger than those of the MW [227]. This has been noted but has found no explanation in the SMoC. That the Andromeda satellites are systematically larger than the MW satellites appears to be qualitatively consistent with SID if Andromeda is more massive than the MW or if the satellites of Andromeda are systematically closer to Andromeda than those of the MW.

It has also been observed that elliptical galaxies in galaxy clusters are systematically larger than isolated elliptical galaxies [72]. Again, this appears to be consistent with the EFE.

However, so far no quantifications of the EFE concerning sizes of galaxies have become available, so that final statements on a verification of the prediction cannot be made. ${ }^{12}$

\subsubsection{Evidence against the EFE}

Hernandez et al. [228] study the statistical properties of very wide binary stars in the Galactic field. If the EFE were active then such binaries, by being immersed in the Galactic external field, should show the Keplerian relative velocity fall-off with separation. But the data suggest the motions of the binary-star companions to conform to SID in contradiction to the EFE. [228] thus finds evidence that Kepler's third law is violated for wide binaries (separation $\gtrsim 7000 \mathrm{AU}$ ) and for the EFE to be suppressed.

A similar behavior is found by Scarpa et al. [229] for the velocity dispersion profile of seven globular clusters which remains flat in their outer regions in disagreement with the expected Keplerian fall-off if the EFE were active. That is, the outskirts of the globular clusters appear to remain in the SID regime rather than showing the EFE-induced suppression of SID. [230] develops self-consistent dynamical models of globular clusters, based on the gravitational theory of [231], to account for this SID behavior.

Further observational constraints on the important EFE are needed to clarify this situation.

\subsubsection{Globular clusters}

Related to the above, it has been noted that if a globular cluster (GC) is of sufficient low density and sufficiently distant then it ought to be in the SID regime [232]. A number of teams have since tested this prediction. So far no clear evidence for SID has been found ( [11]; see also the Ibata/Sanders disagreement: [233]). On the other hand, SID (and the full Milgromian dynamics theory, see Sec. 6.3) is valid for continuum dynamics. A Milgromian-dynamics theory in the collisional

\footnotetext{
${ }^{12}$ Here it is perhaps useful to know that the scientific community actively studying and testing SID/Milgromian dynamics amounts to less than ten persons, in contrast to the hundreds working in the SMoC framework. Asking for even modest research grants to foster work in SID has typically been greeted with rejections.
}

regime, in which two-body encounters and thus the energyequipartition process, play a significant role in the dynamical evolution of a system, has not been formulated yet (see Footnote 12 ).

\subsection{Milgromian dynamics}

It is well known that SID breaks down in the Newtonian regime. The transition from the SID regime into the Newtonian regime is subject to on-going research. [218] studied this transition for the non-relativistic case. By assuming that inertia is linked to the physical processes in the vacuum through a balance between the Unruh radiation and the Gibbons-Hawking background radiation (see also Appendix A in [62] and Sec. 8 here), Milgrom suggests a transition function such that the resulting theory extends from the deep SID regime into the Newtonian regime. It is commonly known as modified Newtonian dynamics (MOND, [10]). Reviews of this field are provided by $[11,58,60,64,234]$ and [20]. In particular, with their major review, Famaey \& McGaugh (2012, [11]) cover various gravitational theories and the possible deeper physics of Milgromian dynamics. The complete Milgromian description of classical gravity has a Lagrangian formulation such that this theory is energy and angular momentum conserving [235]. Minimizing the action leads to the non-linear generalized Poisson equation,

$\vec{\nabla} \cdot\left[\mu\left(\frac{|\vec{\nabla} \phi|}{a_{0}}\right) \vec{\nabla} \phi\right]=4 \pi G \rho$,

where $\phi$ is the potential such that $\vec{g}=-\vec{\nabla} \phi$ (whereby $\overrightarrow{g_{\mathrm{N}}}=$ $-\nabla \vec{\phi}_{\mathrm{N}}$ is the Newtonian acceleration derived from the Newtonian potential sourced by the baryonic matter density $\rho$ and which obeys the Poisson equation $\nabla^{2} \phi_{\mathrm{N}}=4 \pi G \rho$ ). In eq. 14 the function $\mu(x) \rightarrow 1$ for $x \gg 1$ and $\mu(x) \rightarrow x$ for $x \ll 1$ (see [11, 235] for details). This formulation (eq. 14) is not unique, as yet another formulation at the classical level has recently been presented (see $[11,236]$ for details) and implemented in the Phantom of Ramses (POR) simulation code (Lüghausen, Famaey \& Kroupa 2014, [237]).

Simulations of the evolution of galaxies in Milgromian dynamics have been performed showing generally better agreement with the observed galaxies than galaxies modelled in Newtonian dynamics and dark matter [238]. The formation of disk galaxies in growing (phantom) dark matter halos that have no significant merging histories is successful [166]. The observational result that galaxies are simpler than expected if the SMoC were true [61] is consistent with galaxies obeying SID. Such simulations have furthermore demonstrated that mergers are rare because dynamical friction is significantly suppressed given the absence of large and massive dark matter halos, as shown by Tiret \& Combes [238,239]. Galaxies may thus interact with each other repeatedly which can be tested with the longevity of Hickson compact groups (prediction 9.5 in Sec. 9). It will remain to be seen if the peculiar symmetric Local Group structure and the relative orientation of the VPOS and GPoA [91] as well as other dwarf galaxy arrangements nearby the Local Group [184] may follow naturally from a past encounter between the MW and Andromeda in SID [181]. Such an encounter may have produced sufficient debris that 
follow-up encounters between parts of it and the original major galaxies may lead to the symmetrical organized distributions of dwarfs evident in and nearby to the Local Group.

An isolated star is surrounded by its own phantom isothermal dark matter halo, as would be deduced by an observer who interprets the data in terms of Newtonian dynamics. By eq. 10 SID will break down at a radius, $r_{\mathrm{N}}$, at which $g=a_{0}$, i.e. at

$r_{\mathrm{N}}=\sqrt{\frac{G M}{a_{0}}}$.

If $M=1 M_{\odot}$ and since $G=0.0045 \mathrm{pc}^{3} M_{\odot}^{-1} \mathrm{Myr}^{-2}$ and $a_{0} \approx 3.8 \mathrm{pc} / \mathrm{Myr}^{2}$, it follows that each star carries a Newtonian bubble around it with radius $r_{\mathrm{N}} \approx 0.03 \mathrm{pc}$. Thus, only in regions where the star-star separations are $\lesssim 0.03 \mathrm{pc}$ will the system be in the Newtonian regime, while at lower density it will be in the SID regime and will thus show evidence for dark matter. Thus, most of the Universe is in the SID regime.

A note to history: often it is argued that Milgrom's law [10] has been derived from the mass-discrepancy-acceleration correlation discussed in Sec. 6. This is not correct. These correlations were used as tests of Milgrom's original proposal of 1983. Likewise, Milgromian dynamics has not been derived from the BTFR. In actual fact, that low-surface brightness galaxies and gas-rich dwarf galaxies such as DDO210 (baryonic mass $M \approx 5 \times 10^{6} M_{\odot}, v_{c} \approx 15 \mathrm{~km} / \mathrm{s}$ ) lie on the BTFR constitutes a highly significant and successful verification of Milgrom's law in a regime not known to exist in 1983.

A theoretical argument sometimes voiced against MOND is that it has a preferred frame, because it is based on accelerations relative to $a_{0}$. In one formulation of Milgromian dynamics, bimond [50], the acceleration is relative to another metric in the form of the subtraction of two Christoffel symbols. Milgrom's suggestion (1999, [218] that SID may result from a modification of the laws of motion through the quantum mechanical vacuum probably needs a preferred frame indeed, and TeVeS [47] has some preferred-frame effects too. But this does not imply a fundamental obstacle against such theories, since nature may have this property. That there is a preferred frame is evident from the $\mathrm{CMB}$, since the motion relative to the $\mathrm{CMB}$ is measurable. And, Einstein-aether theories (e.g. the review by Eling et al., 2004 [240]) are quite popular despite having preferred frames.

As a final note, a citation from Milgrom [216] on SID, and especially on the symmetry eq. 2 , is important: "(1) it may help put MOND on more sound footings, showing that it need not be imposed as an ad hoc dictate of phenomenology, (2) identifying an underlying symmetry may help extend MOND to non-gravitational systems for which we have no phenomenological guidance, at present, (3) identifying a partial symmetry valid in the deep-MOND regime may lead us to identify a larger symmetry in this regime, or to identifying a symmetry group for the full MOND theory, and this will help constrain the MOND theory itself, and (4) this description is more readily amenable to direct predictions." The symmetry underlying SID may thus be pointing to a new or at least improved understanding of gravitation.

\subsection{Testing Milgromian dynamics}

Milgromian dynamics has, so far, been found to be a remarkably successful despite the many tests that have been tried to disprove it [11]. Perhaps the hardest test has been invented by my research group in Bonn by using GCs (Baumgardt et al. 2005 [232], Sec. 6.2.3). A theory-confidence graph has also been constructed for Milgromian dynamics in Kroupa (2012, [5]). It appears to fare significantly better than the one for the SMoC (Sec. 5.2) because virtually all of the failures of the $\mathrm{SMoC}$ on galactic and galaxy-cluster scales disappear. The literature contains numerous cases of claims against Milgromian dynamics which have, time and again, been refuted or even withdrawn (e.g. on the rotation curve of Holmberg II: [241] as a rebuttal of [242] and the retraction of the original claim [243]; on the rotation curve of M33: [244] which is refuted by [11]).

A clear-cut falsification of Milgromian dynamics would be available if a truly isolated disk galaxy is found with a rotation curve which deviates significantly from the Milgromian one. No such case is known. At the same time, the galaxy population which arises in a universe governed by Milgromian dynamics has not been computed yet, so it is not possible now to perform the same in-depth tests as are available for the SMoC $[5,62]$. At the present it is not known how galaxies form in a Milgromian universe, nor is it known how they evolve over cosmic time. It is hoped that all of this may now become possible with the availability of the POR code by Lüghausen, Famaey \& Kroupa (2014, [237], Sec. 10).

\section{The build-up of stellar populations in galaxies}

The accretion of gas onto galaxies is an important cosmological problem and is inferred largely through the observed galaxy-wide star-formation rates (SFR) which need to be balanced by the in falling material for near-constant star-formation histories. For example, the MW is known to be accreting gas at a rate comparable to the SFR (a few $M_{\odot} \mathrm{yr}^{-1}$ [182]). If dark matter is not present, then the dark halos cannot aid in focussing the gas flows onto galaxies. Observationally deduced gas accretion rates, e.g. through cold filamentary flows (e.g. [208, 245]), thus yield constraints on the effective gravitational potential of the galaxies.

Inferring SFRs is based on various tracers, most of which rely on detecting the massive-stellar population. Assuming a stellar initial mass function (IMF) which is normalized by the observational estimate or the quantity of the tracer (e.g. the $\mathrm{H} \alpha$ luminosity) then allows an estimate of the SFR [246,247]. The time-evolving SFR defines the evolution of a galaxy.

The evolution of galaxies constitutes one of the greatest current research problems in astrophysics (e.g. [151, 248-250]). The many physical processes involved are reviewed by [251] which suggests that evolution driven by mergers between darkmatter halos cannot be the correct theory, a deduction which is consistent with the apparent validity of SID in galaxies (Sec. 6) and the lack of galaxy-galaxy mergers in this effective gravitational framework [238, 239]. Galaxies appear to be selfregulated systems [252] and are much simpler than expected if the SMoC were true, by being populations governed largely by one parameter only [61]. This is particularly demonstrated by the too-small number of late type galaxies with bulges and the need for smooth accretion probably from filaments to fuel the gas supply in galaxies. Late-type star-forming galaxies thus 
constitute the by far dominant ( $\gtrsim 90 \%$ ) population of galaxies with baryonic masses $\gtrsim 10^{10} M_{\odot}$ ( [152], see also fig. 4.14 in [153]) and their formation and evolution is quite well understood as long as the dynamics of the putative particle dark matter is suppressed and they are allowed to accrete baryons smoothly $[166,251]$. However, observations do show that in the past late-type galaxies had more complex morphology with signs of interactions increasing with increasing redshift (e.g. $[152,253])$ and/or signs of internal instabilities such as evident in the chain galaxies at redshifts between 0.5 and $2[254,255]$. Noteworthy in this context is that accounting for the thick disk of the Milky Way through clustered star-formation (Sec. 7.3) predicted the Milky Way to have been a chain galaxy about 11 Gyr ago [256].

Elliptical or early-type galaxies with baryonic mass $\gtrsim 10^{10} M_{\odot}$ have been observationally deduced to form rapidly early on and not to build-up from mergers over cosmic time of late-type galaxies $[57,155,190,257,258]$. This remarkable result had been suggested already by the pioneering work of Matteucci (1994, [259]), being later confirmed by [257, 258, 260]. Requiring elliptical galaxies instead to conform to merger-driven buildup requires the postulate that they have a merger history with early-type (gas-poor) galaxies. But such a population of galaxies remains undiscovered, unless the merging sub-units are faint early-type dwarf galaxies or most of the merging has finished by 6 Gyr ago. Since then the population of elliptical galaxies does not seem to have evolved [152], which would make the present cosmological epoch special. [261] explicitly test this scenario and rule it out.

The stellar populations which arise in the galaxies are the most important tracers that allow an observational approach to galaxies. But how do these build-up?

Most models of star formation and feedback in galaxies have been assuming the stellar initial mass function (IMF) to be invariant. The theoretical and observational evidence has however shown this to be incorrect. Before turning to the observationally confirmed variation of the IMF in Sec. 7.4, the hottopic of a bottom-heavy IMF in elliptical galaxies is briefly discussed.

\subsection{A bottom-heavy IMF in elliptical galaxies?}

The cooling-flow problem in galaxy-clusters led to the suggestion that the X-ray emitting cooling gas may be forming low mass stars in the central elliptical galaxies as these would not be observable as star-forming systems. Stellar gravitysensitive spectral absorption features in the $\mu \mathrm{m}$ spectral region were proposed to test the notion that such elliptical (E) galaxies may have a large population of low-mass stars (Kroupa \& Gilmore 1994, [262]). Applying the dwarf-sensitive spectral features $\mathrm{Na}$ I, Ca II, and $\mathrm{FeH}$ in the $\mu \mathrm{m}$ spectral range uncovered the signature of stellar mass functions heavily dominated by low-mass stars. The work of van Dokkum \& Conroy [263] (see also [264]) unambiguously showed these spectral indicators to strengthen with increasing E galaxy mass such that massive E galaxies would have had stellar IMFs much steeper than the Salpeter index. But, as pointed out by [265], there has not been a theoretical prediction of such extreme IMFs; theory ever only predicted the IMF to become top-heavy with increasing temperature or decreasing metallicity and in star-bursting systems. The IMF results on E galaxies are thus highly puzzling but also very interesting. In particular, a bottom-heavy IMF would pose a challenge for understanding the observationally deduced rapid chemical evolution of E galaxies such that hybrid models of a top-heavy galaxy-wide IMF in the initial star burst evolving to a bottom-heavy galaxy-wide IMF over an extended time of lower-intensity star formation would be required $[260,266]$.

The notion that the IMF becomes extremely bottom-heavy in massive $\mathrm{E}$ galaxies has been tested by observing the closest massive E galaxy with a strong-lensing signal [267]. This lensing signal allows the mass of the E galaxy to be constrained, and it is found to yield a $M / L$ ratio consistent with a canonical IMF (which is botom-light compared to a Salpeter IMF) at the age of the galaxy. This same galaxy has, however, the strong spectral absorption bands that appear to imply a strongly bottom-heavy IMF. Such a strongly bottom-heavy IMF would be inconsistent with the measured amount of mass within the strong lens. Thus, these spectral features must have a different origin, i.e., they cannot stem from a large population of low-mass stars. An independent test of the bottom-heavy IMF in E galaxies has been performed by [268] by testing whether the number of low-mass X-ray binaries (LMXBs) varies with E-galaxy mass as it should if the relative number of massive to about solar-mass-stars in the IMF changes. Their results are not consistent with the IMF becoming strongly botom-heavy in more massive E galaxies. The origin of the dwarf-star-sensitive spectral features is suggested by [269] to come from a certain type of evolved binary stars being disrupted more in lowermass $\mathrm{E}$ galaxies than in massive $\mathrm{E}$ galaxies which have lower stellar number densities in combination with such stars missing in the spectral libraries used to synthesize the models that, due to the omission of such stars, imply strong dwarf-star populations.

In conclusion, although the spectral signatures do seem to be convincing evidence, it appears that the independently used constraints do not support the notion that the IMF becomes increasingly and significantly bottom-heavy in increasingly massive $\mathrm{E}$ galaxies. The trend of increasing dynamical $M / L$ ratio with increasing E-galaxy mass $[66,270]$ may be explained by an increasingly top-heavy galaxy-wide IMF through the presence of an increasing fraction of stellar remnants in more massive E galaxies. This is consistent with the IGIMF (Sec. 7.5) becoming top-heavy in star-bursting galaxies and with the chemical enrichment histories of massive $\mathrm{E}$ galaxies, as discussed below. However, this result must also be tested independently by constraining the mass via strong lensing and the relative number of remnant and main sequence stars using LMXBs.

\subsection{Is star formation stochastic or self-regulated?}

There are two fundamentally different approaches to account for the emergence of stellar populations in galaxies. The one is to assume that star formation is a stochastic process such that the distribution of stellar masses, i.e. the IMF, is a probability density distribution function (PDF), which is usually assumed to be invariant. Complete logical consistency would follow if the form of the IMF was stochastically variable. This is not a well-motivated physical description but is simplest to model. The other is to assume that the IMF is subject to constraints posed by local physical conditions such that the process of 
star formation is not stochastic, but is self-regulated instead in galaxies that are in dynamical equilibrium. This approach is well-motivated physically but is harder to formulate since the underlying laws need to be discovered. It opens the avenue for studies with systematically varying IMFs.

Dynamical population synthesis [271] and with the advent of infrared imaging capabilities and high-resolution $(<1 \mathrm{pc})$ observations of star-forming molecular clouds have shown that star-formation occurs in compact embedded clusters (ECs). The review by [272] established this and that the ECs are distributed by mass according to a power-law embedded star-cluster mass function (ECMF). The build-up of galaxies therefore occurs from such structures.

The application of the above notions on stochastic versus constrained star formation to ECs leads to the two following possibilities: The emergence of ECs can be modelled either as a stochastic process such that the ECMF is assumed to be a PDF. Or the ECMF is subject to constraints posed by local physical conditions such that the process of clustered star formation is not stochastic.

\subsection{Embedded clusters: the fundamental building blocks of galaxies}

An EC forms from a molecular cloud core on a pc-scale or less and within a Myr or less, with a star-formation efficiency smaller than $50 \%$ and probably close to $33 \%$. The EC expands significantly [273] after self-removal of its residual gas through the feedback of its own young stars thereby dispersing a large fraction of them into the galactic field. This has been computed for ECs on a stellar mass scale of $10<M_{\mathrm{ecl}} / M_{\odot}<100$ [274], for $M_{\mathrm{ecl}} \approx 10^{3} M_{\odot}$ [275], for $M_{\mathrm{ecl}} \approx 10^{4} M_{\odot}$ [276], and for $M_{\mathrm{ecl}} \approx 10^{5} M_{\odot}$ [277]. Observations with the Hershel Space Observatory have shown the groups of stars and clusters to form in molecular cloud cores along thin filaments with radii or cross sections near $0.1 \mathrm{pc}$ [278], which is consistent with the independently obtained radius-mass relation of embedded clusters [279]. The masses of embedded clusters range from groups of a dozen binaries $\left(\approx 10 M_{\odot}\right.$, which appear to an observer as "isolated" star formation) to an upper limit which is unknown but may be as large and perhaps larger than $10^{7} M_{\odot}$ [279-281].

Thus, the formation of the stellar population of galaxies occurs, over time, through the successive addition of stellar populations individually born in star-formation events which are correlated in space and time. In this sense we arrive at the lego principle [282] according to which stellar populations in galaxies are obtained by adding together the individual populations formed in embedded clusters. The "lego principle" is visualized in Fig. 10.

These "fundamental building blocks" (i.e. the embedded clusters) are observed to obey empirically discovered laws which are simple. When considered these lead to a computable, predictive description of young populations of stars in galaxies. For example, the thickening of thin galactic disks over time can be derived from this lego principle [256], the binary population in different galaxies can be predicted [286], and the stellar IMF and its variation with sar-formation rate of a whole galaxy can be calculated $[265,281]$.

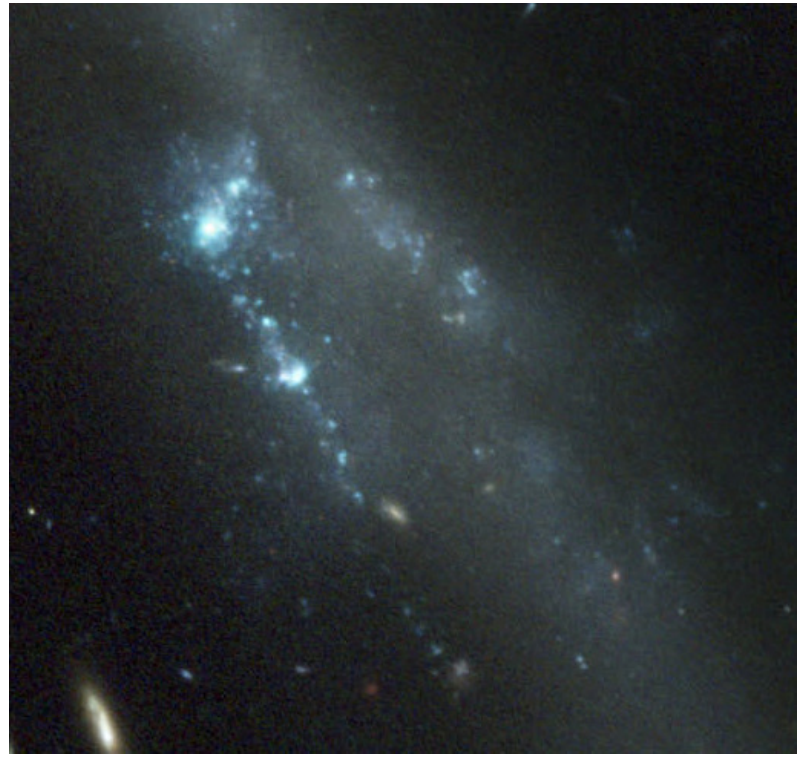

Fig. 10. A close-up of one of the TDGs in the tadpole galaxy shown in Fig. 4. This demonstrates the "lego principle" [282] according to which star formation in galaxies occurs in embedded star clusters which expel the residual gas not used up in the star-formation process and become visible as very young stellar populations seen here as the small faint blue dots. [283] estimate the mass of the TDG in the upper left part of the image to be about $1.3 \times 10^{6} M_{\odot}$ with a radius of about $160 \mathrm{pc}$ and age of about 4.5 Myr. This star-cluster complex may be a progenitor of much older objects as it typically evolves into spheroidal dwarf galaxies, depending on the details of the orbit and the tidal field [77,284]. Compare this to the masses $\left(<10^{7} M_{\odot}\right)$ and radii $(\approx 250 \mathrm{pc},[285])$ of the MW and Andromeda dSph satellite galaxies. The scale of the figure is approximately $8.6 \mathrm{kpc} \times 9.1 \mathrm{kpc}$ (vertical $\mathrm{x}$ horizontal). 


\subsection{The empirical laws of star formation}

The empirical laws can be summarized as follows (see [281] for a thorough description): the stellar IMF in each EC is forminvariant; the mass of the most massive star, $m_{\max }$, within the $\mathrm{EC}$ is a function of the mass of the EC, $m_{\max }=\mathrm{K}_{1}\left(M_{\mathrm{ecl}}\right)$ (the $m_{\max }-M_{\text {ecl }}$ relation); the ECMF is a power-law distribution function with index $\beta(\beta=2.35$ is the Salpeter index); the most-massive EC forming in a population of ECs is a function of the galaxy-wide star formation rate (SFR), $M_{\mathrm{ecl} \text {, } \max }=\mathrm{K}_{2}(S F R)$ (the galaxy-wide $M_{\mathrm{ecl}, \max }-S F R$ relation).

The parameters entering the empirical laws are constrained using observational data of well-resolved star-formation events. Thus, the stellar IMF is well-known to be the canonical form (i.e. a two-part-power law with Salpeter-Massey index $\alpha_{2}=$ 2.3 for stars more massive than $0.5 M_{\odot}$ and with $\alpha_{1}=1.3$ for less massive stars, [256,265,287]). It is particularly important to be aware of the biases that enter observational deductions on the shape of the IMF [265]: binary star populations evolve on a cluster dynamical time-scale and may wrongly suggest IMF variations if not corrected for, massive stars are efficiently dynamically ejected from clusters such that they are missing from the star counts, the expulsion of residual gas during star formation unbinds low-mass stars in mass-segregated clusters leading to wrong inferences about the IMF shape unless this is taken into account. The $m_{\max }-M_{\text {ecl }}$ relation, $m_{\max }=\mathrm{K}_{1}\left(M_{\mathrm{ecl}}\right)$, has been established from observed ECs [288] and $M_{\text {ecl,max }}=\mathrm{K}_{2}(S F R)$ is constrained by extragalactic star-forming galaxies [289]. Furthermore, the functions $K_{1}$ and $K_{2}$ readily follow from mass-constraints on the respective distribution functions (the IMF and the ECMF).

Accounting for the biases, the observational constraints suggest that the stellar IMF becomes top-heavy when an EC surpasses the critical mass of $2.7 \times 10^{5} M_{\odot}$ which obtains from a critical level of star-formation rate density during the formation of an individual massive EC. This evidence for top-heavy IMFs in massively star-bursting clusters appears from four entirely independent data sets: (i) the elevated dynamical $M / L$ ratios of UCDs [290,291], (ii) the higher incidence of LMXBs in UCDs [292], (iii) globular clusters in the Milky Way which have "damaged" present-day mass functions of low-mass stars [280], and (iv) the element anti-correlations observed in massive globular clusters [293]. This recently discovered tendency of the stellar IMF to be increasingly top-heavy with increasing star-formation rate density within (extremely massive) ECs is the key to understanding the independently obtained evidence from elliptical galaxies and from large surveys of star-forming galaxies for top-heavy galaxy-wide IMFs at high SFRs ( [258, 259, 294-299], see [281] for a review of the evidence including the cosmological star formation history). The top-heavy stellar IMF may result in molecular clouds that are cosmic-ray heated [300,301].

The only parameters not constrained by observation independently of the IGIMF framework are $1.5 \lesssim \beta \lesssim 2.5$ and the minimum embedded-cluster mass in the population of embedded clusters, $M_{\text {ecl,min }} \gtrsim 10 M_{\odot}$, both of which enter the ECMF. These two parameters may be varied for improved fits, and they are found to obey systematic variation with the galaxywide SFR [281].

\subsection{Star formation theories}

From hereon the following three classes of theories will be discussed:

A: Purely stochastic star formation such that the IMF is an invariant PDF, and the ECMF does not play a role. Stars may be clustered randomly into ECs without conditions which themselves form a random population.

This is the simplest method to model the forming stellar population and is also the most popular. A star-formation event in an analytical model of galaxy evolution consists typically of a fully populated IMF which may thus have fractions of massive stars and the corresponding fractional feedback energy. If variations of the IMF are admitted it is either necessary to assume systematic variation subject to a rule while the formation of individual stars would be stochastic, which leads to an inconsistent approach. Or, the variation of the IMF would need to be stochastic as well, which leads to consistency issues concerning the shape of the IMF at a given drawing of a population.

B: Stochastic but clustered star formation such that the IMF is a PDF but it must fulfill constraints posed by the mass of an embedded cluster and the ECMF is also a PDF.

This is the primitive version of the IGIMF theory. According to this description star formation in a galaxy always occurs in embedded star clusters, but these may be made up of individual single stars (e.g. an EC of mass $100 M_{\odot}$ may be comprised of a single star of mass $\left.100 M_{\odot}\right)$.

C: Regulated star formation such that neither the IMF nor the ECMF are PDFs with stellar and cluster masses allowed to be sampled over their entire ranges $\left(\approx 0.1-150 M_{\odot}\right.$ for stars, $\gtrsim 10 M_{\odot}$ for ECs). This is the IGIMF theory as proposed by Weidner \& Kroupa [302,303] for an invariant stellar IMF, while the extension to allow for the systematically varying IMF as obtained from data on ultra-compact dwarf galaxies and on globular clusters is formulated by $[265,281]$. The above empirical laws of star formation enter this description.

According to this description an EC contains a forminvariant IMF up until a most massive star, $m_{\text {max }}$, such that the mass of the stellar population equals the mass of the EC yielding the correct function $m_{\max }=\mathrm{K}_{1}\left(M_{\mathrm{ecl}}\right)$. A star-formation epoch consists of a full ECMF, the most massive star cluster of which scales with the galaxywide star formation rate yielding the correct function $M_{\mathrm{ecl}, \max }=\mathrm{K}_{2}(S F R)$. The integrated galactic initial mass function of stars (the IGIMF), i.e. the galaxywide IMF, is calculated by integrating over all newly formed ECs with their individual stellar IMFs. An extreme version of the IGIMF formulation assumes both the IMF and the ECMF to be optimally sampled distribution functions [265].

The three procedures lead to very different results on galactic scales, because a galaxy model will have a different history of chemical enrichment and feedback energy release, and the 
photometric and spectral emission will differ (e.g. [107, 189, 190, 304, 305]). Bekki (2013, [306]) has incorporated, for the first time, a galaxy-wide variable IMF based on the empirical laws of star formation (Sec. 7.4) showing that chemical enrichment proceeds faster in massive galaxies which form a smaller mass in stars than if the IMF were universal.

The differences between the different approaches become most apparent for star-forming dwarf galaxies. With theory A (purely stochastic star formation) an ensemble of dwarf galaxies will have, for example, the same average ratio of $\mathrm{H} \alpha$-to-UV emission as massive galaxies, while in theory $\mathrm{C}$ (regulated star formation) dwarfs will have a significantly lower ratio. This comes about because dwarf galaxies have low SFRs [307] such that only low-mass ECs form which do not contain massive stars. The following result is obtained [281]:

GALAXY-WIDE VARIATION OF THE IMF: According to the IGIMF theory dwarf galaxies have top-light IGIMFs, while massive galaxies have top-heavy IGIMFs.

\subsection{Tests of star formation theories}

Table 1 tests the three theories sevenfold against observational data. It follows that no tests are passed by theory A, a few tests are passed by theory B while all are passed by theory $\mathrm{C}$. The modest success of theory B has been used by some groups to erroneously argue that star formation is stochastic. Such research groups fail to take into account the other constraints evident in Table 1 . In reality, theory $\mathrm{C}$ accounts for the star formation observed locally in the MW and at the same time for the chemical and photometric tracers on galaxy-wide scales. This approach allows a computable evolution of the SFR with cosmic evolution as it accounts also for the observed evidence for top-heavy galaxy-wide IMFs in galaxies with high SFRs.

Scatter in the data, e.g. amongst dwarf galaxies, can be erroneously interpreted by some researchers to be evidence for stochastic star formation, especially in combination with a compromised or oversimplified analysis (see e.g. [308]). But scatter in observational data arises due to measurement uncertainties as well as from variations in the SFRs due to galaxyinternal processes (e.g. "breathing" or "gasping" modes) or from external perturbations (e.g. [309]). For example, the scatter in observational data around the theoretical $m_{\max }=\mathrm{K}_{1}\left(M_{\text {ecl }}\right)$ relation can be ascribed entirely to the observational uncertainties such that it cannot be excluded that there is no intrinsic scatter in the $m_{\max }-M_{\mathrm{ecl}}$ relation [288].

\subsection{The main sequence of galaxies}

That theory $\mathrm{C}$ passes all tests leads to a simple and computable understanding of star-formation on galaxy scales, being consistent with the observational result that galaxies appear to be simple objects as already concluded by Disney et al. from analyzing a major observational sample [61]. A significant implication of the above is that dwarf galaxies have a deficit of massive stars such that the $\mathrm{H} \alpha$ tracer leads to a wrong normalisation of the SFR if it is assumed (wrongly) that the IMF remains fully sampled. In truth, dwarf galaxies may have SFRs up to two orders of magnitude larger than the standard calibra-

\begin{tabular}{l|cccc} 
Test & Theory & $\mathrm{A}$ & $\mathrm{B}$ & $\mathrm{C}$ \\
\hline I & L1641 & $\mathrm{N}$ & $\mathrm{N}$ & $\mathrm{Y}$ \\
II & $m_{\max }=\mathrm{K}_{1}\left(M_{\mathrm{ecl}}\right)$ & $\mathrm{N}$ & $\mathrm{N}$ & $\mathrm{Y}$ \\
III & very small dispersion of $\alpha_{3}$ values & $\mathrm{N}$ & $\mathrm{N}$ & $\mathrm{Y}$ \\
IV & maximum cluster mass & $\mathrm{N}$ & $\mathrm{N}$ & $\mathrm{Y}$ \\
V & $\mathrm{H} \alpha / \mathrm{UV}$ & $\mathrm{N}$ & $\mathrm{Y}$ & $\mathrm{Y}$ \\
VI & mass-metallicity relation & $\mathrm{Y})$ & $(\mathrm{Y})$ & $\mathrm{Y}$ \\
VII & observed variation of IMF & $\mathrm{N}$ & $\mathrm{N}$ & $\mathrm{Y}$
\end{tabular}

Table 1. Testing theories A, B and $\mathrm{C}$ on observational data: Test I: Molecular cloud L1641: low-density population of $\approx 1600$ stars but no O stars [310]. This cannot be accounted for with stochastic star formation. Test II: $m_{\max }=\mathrm{K}_{1}\left(M_{\mathrm{ecl}}\right)$ relation: small observed scatter of data rules out stochastic sampling from an invariant IMF [288]. The growth of embedded clusters follows such a relation [311]. Test III: for $m>3 M_{\odot}$ the observationally derived IMF power-law indices show a very small dispersion about the Salpeter value which is in conflict with random sampling of stars from an invariant IMF ( [312]; especially sec. 9.5 in [265]). Test IV: The maximum masses of young star clusters in the galaxy M33 show a significant decrease with increasing radial distance ruling out stochastic star formation [313]. A similar result is found by [314] in the variation of the most-massive young cluster with galaxy SFR. Test V: H $\alpha / \mathrm{UV}$ flux ratio for dwarf galaxies decreases systematically with decreasing SFR [307] - this can be explained by theory B (Pflamm-Altenburg, priv. comm.) but has been predicted by theory C [315]. Test VI: Galaxies have an increasing metallicity with increasing mass which can only be explained with fine-tuned metallicity-dependent winds for theories A and B but follows naturally in the IGIMF theory [189, 190]. Test VII: Multiphotometric-band surveys of thousands of late-type galaxies by independently working teams have uncovered strong evidence of a systematic change of the galaxy-wide IMF with increasing galaxy mass and increasing SFR [297-299,307]. Pioneering deductions of this had already brilliantly been achieved by Matteucci (1994, [259]) being confirmed by [258]. This is incompatible with stochastic/random sampling of an invariant IMF but has been predicted qualitatively by the IGIMF theory [303] and has been quantitatively accounted for by the most modern version which includes top-heavy IMFs in massive star-bust clusters [281]. 
tions imply. Late-type dwarf galaxies may thus be as efficient in forming stars as are major disk galaxies:

IMPORTANT: Theory $\mathrm{C}$ (the IGIMF theory) thus leads to the result that the gas-depletion time-scale is $\tau_{\text {gas }} \approx 3 \mathrm{Gyr}$ for all star-forming galaxies [316].

Since $\tau_{\text {gas }}=M_{\text {gas }} / S F R$ and for a gas fraction per stellar mass, $\eta=M_{\text {gas }} / M_{*}$, this result implies for the specific SFR, sSFR,

$\mathrm{sSFR} \equiv \frac{S F R}{M_{*}}=\frac{\eta}{\tau_{\text {gas }}}$,

such that $\mathrm{sSFR} \approx 10^{-10.18} \mathrm{yr}^{-1}$ for $\eta=0.20$. Thus, by analyzing galaxies within the IGIMF theory it follows that the galaxies are on a main sequence, as is observed $[151,317]$. Note that the assumption $\eta=0.2$ (20\% of the stellar mass) may not hold for late-type dwarf galaxies. Dwarf galaxies are observed to have increasing $\eta$ with decreasing stellar mass such that sSFR would be decreasing with increasing galaxy stellar mass,

$\operatorname{sSFR}=\frac{\eta\left(M_{*}\right)}{\tau_{\text {gas }}}$.

Applying the IGIMF theory to the observed fluxes from the dwarf galaxies, Pflamm-Altenburg \& Kroupa (2009, [316]) show that such dwarfs would have acquired up to 10 or more times the stellar mass in late-type stars than an invariant standard IMF would imply, such that the baryonic gas fractions, $f_{g}=$ $M_{\text {gas }} /\left(M_{\text {gas }}+M_{*}\right)=\eta /(1+\eta)$, reported in [318] may need some revision.

The IGIMF-analysis by [316] implies that late-type galaxies may be replenishing their gas supply on this same time-scale, $\tau_{\text {gas }}$, and that the observed problem with stellar-mass buildup time are solved within the IGIMF theory. This in turn has implications for understanding the cosmological matter cycle, but is not evident in the standard, stochastic descriptions based on dark-matter merging trees.

IN CONCLUSION: galaxies follow a simple gravitational law as well as a simple star-formation law. They appear to be largely self-regulated systems through the SFR-dependent IGIMF.

Self-regulated star formation in galaxies has been introduced by Koeppen, Theis \& Hensler (1995, [252]), and emerges in simulations of the smooth formation of Milky Way type galaxies (Samland \& Gerhard, [166]). How self-regulation emerges within the IGIMF theory, which leads to a stronger coupling between the SFR and feedback energy, is studied with the pioneering work by Plöckinger et al. ( $[107,319]$ ), who incorporated a SFR-dependent IGIMF formulation into gas-dynamical simulations of galaxy formation with feedback. This work demonstrates that dwarf galaxies can build-up a larger stellar mass than is possible with a universal IMF. Previous models on TDGs applying the IGIMF theory have been computed by Recchi et al. (2007, [121]). Recchi et al. (2009, [190] has shown that the alpha-element abundances in early-type galaxies are better and more naturally matched within the IGIMF theory, and that the need for downsizing is relaxed somewhat.
That the mass-metallicity relation of galaxies emerges naturally within the IGIMF theory has been shown by Köppen et al. (2007, [189], see also Fig. 8). The observational data thus appear to indicate that self-regulated, SFR-dependent IGIMF description of galaxy evolution may be more realistic than an invariant IMF. This is nicely consistent with the independent evidence from galaxy surveys which suggest a systematic change of the galaxy-wide IMF with SFR (Sec. 7.4, Tab. 1).

Excursions from self-regulated evolution, e.g. through galaxygalaxy encounters, and a possible initial spread of galaxy-formation times [151] lead to a dispersion of properties about the main sequence, which are easily accommodated with the SFRdependent IGIMF theory rather than being evidence for stochastic star formation. The dispersion is indeed observed to be remarkably small [151], in agreement with the conclusions reached previously [61].

\section{A conservative cosmological model?}

If cold or warm dark matter particles do not exist, then the $\mathrm{SMoC}$ is ruled out as a consequence. Does an alternative and realistic cosmological model exist?

A Milgromian-dynamics-based cosmological model has indeed been computed. The CMB can be accounted for well, as shown in 2009 by Angus ( [320], see also [321]). Structure formation remains a computational challenge for this model which adopts a hot big bang and Einstein's GR to describe the cosmological dynamics of the expansion at all times as well as the growth of perturbations until recombination. The model also requires dark energy and a hot dark matter component in the form of about $11 \mathrm{eV}$ sterile neutrinos [322], which can be linked to the SMoPP through neutrino oscillations though. It has a flat geometry and is thus very similar to the SMoC, except that the problems with cold or warm dark matter discussed in the previous sections do not arise. Computations of structure formation in Milgromian dynamics are compromised not only by the computational challenge posed by non-linear gravitational dynamics but also by the matter being baryonic rather than non-interacting, as is the case in the much easier SMoC computations of structure formation dealing only with exotic dark matter and linear equations of motions. Available results based on assuming hypothesis 0ii suggest structures to grow more rapidly than in the SMoC $[322,323]$. Issues that arise with the CMB that have been pointed out by [324] have thus partly or wholly been adressed (see also the major review [11]). The dynamics in galaxy clusters, including the Bullet cluster, are accounted for well with the sterile neutrinos making up the small amount of missing mass in Milgromian dynamics [325].

The following may be stated: the "Angus cosmological model" is much more conservative than the SMoC because:

1. It does not change GR.

2. It does not invoke exotic particles.

3. The sterile neutrinos can be linked to the standard model via the neutrino oscillations.

4. Milgromian dynamics for the formation of structures (and dynamics thereof) can be viewed as simply arising as a vacuum effect (Sec. 6.3), rather than changing GR. The 
modification would only be effective at low accelerations due to the presence of the vacuum acting as a lower inertial resistance at very low accelerations.

The last point is the most contentious one, as it implies a particular interpretation of Milgromian dynamics and SID as being due to quantum-mechanical properties of the vacuum. This idea was proposed by Milgrom (1999, [218]), but does not constitute a proper theory with general covariance. It provides a direction and an intuition, something that has been missing from theoretical physics for quite some time.

If Milgromian dynamics arises due to the vacuum, then how can one understand the motion of a star in the SID regime while its constituent matter, its atoms, are embedded in a strong gravitational field? If the underlying fundamental theory can be cast in terms of a modified Poisson equation of Bekenstein \& Milgrom (1984, [235], see their section IV for a discussion of the present issue) then the centre of mass motion of a star can be shown to follow Milgromian dynamics despite its interior being in a self-generated strong field. This works because the force on a body (a star) made of smaller bodies (atoms) can be written as an integral over any surface surrounding the body (excluding all other bodies). One can take the integral on a surface surrounding the star far from the star itself, and show that it is proportional to the total mass of the star, as long as this mass is small compared to that of the galaxy. Note that exactly the same already happens with GR in the strong field and in Newtonian dynamics. Consider a binary BH orbiting a Newtonian galaxy. Each BH is in a very strong field and calculating the exact orbit of each black hole in the three-body system galaxy- $\mathrm{BH}-\mathrm{BH}$ is a complicated general relativistic calculation. But the center of mass of the binary BH obeys Newtonian dynamics in the field of the galaxy. This analogy is made clearer by replacing GR by Newtonian dynamics and Newtonian dynamics by Milgromian dynamics resulting in the same situation for a star and its atoms. Returning to the vacuum notion, each atom can be in a strong field and move according to Newtonian dynamics (or GR) in the frame of the star, but if the centre-of-mass of the star is in a weak gravitational field, then the star orbits according to Milgromian dynamics. As this discussion demonstrates, Milgromian dynamics provides a rich research field for theoretical and mathematical physicists.

As a final note, it is to be pointed out that the existence of "dark energy" could have been predicted by Milgrom by virtue of his argument of 1999 [218]: By proposing that the transition from the Newtonian regime to the SID regime in the weak gravitational regime comes from the quantum mechanical properties of the vacuum, it would have been possible to compute the energy density of the vacuum given observational constraints on $a_{0}$ from the baryonic Tully-Fisher relation or from rotation curves of galaxies. With this vacuum energy in hand, it would have been possible to predict the accelerated expansion of the Universe. Remember that the underlying model is the very conservative Milgrom-based model by Angus [321], i.e. GR is not changed but SID arises as a vacuum effect [218].

But how would the significant local matter under density (Fig. 1) fit-in here? This would need to be calculated along the principles given by e.g. Wiltshire [215]. But in the conservative model the under density would only be associated with baryonic matter and not with a lack of local dark matter, such that the effect on SNIa redshifts may be reduced. That is, the vacuum would nevertheless possibly lead to late-time acceleration. As discussed above, the conservative model appears to lead to more structure on large scales, and this may, qualitatively at least, be consistent with the existence of fluctuations in the matter density as is observed locally. Nevertheless, the assumptions on homogeneity and isotropy inherent in the Angus model do need to be analysed in future work.

As a final comment, the inquisitive reader may be interested to consider the discussion of the CMB in terms of its possible physical properties and origin provided by [326].

\section{Predictions}

The contents of the text above suggest that the astrophysical evolution of galaxies may require major revision. While simulations need to be performed allowing for self-regulated galaxy evolution within the SID and the IGIMF theoretical frameworks, a few predictions can already be stated which ought to be open to experimental scrutiny.

Before turning to new predictions, it is worth remembering that once exotic DM is abandoned, successful predictions seem to emerge. A particularly noteworthy prediction of a Herculestype dSph without DM was obtained by modelling the longterm dynamical evolution of TDGs (Kroupa 1997, [111]). This turned out to match observed dSph and UFD satellit galaxies quite well [178]. The models (see also [114, 115, 179]) of ancient TDGs have shown that the TDGs loose stars at every periastron passage until only about $1-10 \%$ of the original population remains in a quasi-stable remnant with a highly non-isotropic phase space distribution function. An observer applying the usual models for virial systems then incorrectly deduces large dynamical $\mathrm{M} / \mathrm{L}$ ratios. The remnants are not satellite galaxies in the process of disruption.

The Hercules satellite galaxy was discovered a decade later [327] and has the near-exact morphological, luminous and kinematical properties as the model of 1997 (fig. 6 in [62]). Another case may be Bootes III which also resembles the TDGremnant model. Grillmair (2009, [328]) discovered this stellar overdensity to have a complex sub-structured morphology (fig. 10 in [328]) which resembles the DM-free models of ancient remnant TDGs. This is an important point valid for the other satellite galaxies as well: Kroupa (2012, [5]) stresses that given the large velocity dispersion observed within the satellite galaxies and their large distances from the MW, "any internal sub-structure would phase-mix away within 100 Myr. Given their ages of about $10 \mathrm{Gyr}$, it follows that the satellites ought to appear as smooth and symmetric as GCs." The measured velocity dispersions therefore cannot be due to the satellites being immersed within their own DM halos. Grillmair and [329] suggest that Bootes III may be a remnant satellite with a long extension along the line of sight, in agreement with the models of Kroupa (1997, fig. 12 in [111] and figs. 16 and 18 in [114]).

Furthermore, without exotic DM an entirely new dynamical deceleration process for satellite galaxies emerges: discovered for the first time by Yang et al. (2014, [179]), a gas-rich dwarf galaxy which orbits past a major galaxy with coronal gas may have its ISM stripped through ram-pressure. If the ISM is sufficiently heavy it's systematic displacement behind the stellar component leads to "ram-friction" on the stellar component; 
the dwarf galaxy decelerates and may be captured by the major galaxy despite the absence of dynamical friction on a DM halo.

These predictions and theoretical discoveries were obtained in Newtonian dynamics, which is not consistent with abandoning DM. However, they demonstrate above all that the large observationally deduced dynamical $\mathrm{M} / \mathrm{L}$ ratios of $\mathrm{dSph}$ and UFD satellite galaxies do not unequivocally imply them to be DM dominated. For consistency with abandoning DM, the above simulations need to be re-done in Milgromian dynamics. This has not been possible until recently due to the lack of appropriate Milgromian dynamics simulation codes. Early results are available suggesting improved fits of the Milgromian satellites with the observed ones [330], although the details of the binary populations within the satellite galaxies which affect the observed velocity dispersions need to be taken into account [221,331]. The POR code [237] will become an important simulation tool for this purpose, but it is already apparent that the EFE (Sec. 6.1) leads to the satellite loosing its phantom dark matter halo as it approaches periastron such that it is likely to be disrupted more easily than a Newtonian system, although the phantom dark matter halo reappears as the satellite orbits past periastron and towards apoastron. The EFE may thus lead to a critical distance from the MW within which dwarf satellite galaxies are disrupted. It is observed that the satellite population appears to largely extend beyond about $50 \mathrm{kpc}$ suggesting this to be the critical distance for the MW, but quantitative work needs to be done to asses the impact of the richness of Milgromian dynamics on the satellite population.

Other remarkable predictions concern galactic rotation curves: returning to the Gedankenexperiment "Challenge for Theory" in Sec. 2, it is true that the rotation curve of every late-type galaxy for which the spatial baryonic matter content is known, Milgromian dynamics allows the rotation curve to be predicted without a free parameter.

\subsection{Prediction: the number of MW satellite galaxies}

There is today no doubt any longer that the VPOS is established to be a physical property of the MW galaxy (Sec. 5.1.1). It will nevertheless be necessary to search for more satellite galaxies even though finding fainter members will not affect the fact that the at present brighter ones are distributed within a mass-segregated VPOS [5]. If the VPOS is an ancient structure such that it is phase-mixed then the number of satellite galaxies ought to be symmetrically distributed about the MW. The north Galactic hemisphere is known today to have in total 19 $\mathrm{dSph}$ and UFD satellite galaxies (Fig. 2, this includes Crater or PSO J174.0675-10.8774). Until the present day no additional satellites have been found significantly off the VPOS. If the population of satellites northwards of the Galactic disk is complete, then the southern Galactic hemisphere should have in total about 19 satellites as well. Nine are already known (Fig. 2). This leads to the prediction made here for new satellites with luminosities in the range of the existing satellites [285]:

Assuming the VPOS to be a phase-mixed and thus ancient structure, about 10 additional satellite galaxies should be found in the southern Galactic hemisphere.

Alternatively hundreds of dark-matter ultra-faint satellite galaxies have been predicted within the framework of the SMoC to surround the MW spheroidally ( $[99,211]$, Sec. 3.1).

However, even in the unlikely event that this were to be the case, the observed fact is that the VPOS is made of the brightest satellite galaxies such that the models based on dark matter would have to account for why the most luminous satellites would be in a disk of satellites (Sec. 5.1.1). None of the existing research papers working within the SMoC framework address this issue (e.g. the most recent land-mark simulation of the galaxy population by Vogelsberger et al. (2014, [167]).

\subsection{Prediction: proper motions of MW satellite galaxies}

Of the known 28 satellite galaxies that are found within about $270 \mathrm{kpc}$ of the centre of the MW, 11 have proper motion measurements. Of these, 9 are found to be orbiting within the VPOS within the uncertainties. Leo I is on a very radial orbit, and Sgr may have been diverted onto an energetic orbit [139] and it is now on a near-perpendicular orbit to both the MW disk and to the VPOS (Fig. 2). This state-of-affairs allows the proper motions of the remaining known satellite galaxies to be predicted:

Assuming the satellite galaxies to orbit within the VPOS, the proper motions of almost all satellite galaxies of the MW can be predicted. These predictions are available in [92].

Alternatively the satellite galaxies that do not have measured proper motions would have random motions.

However, even in the unlikely event that this were to be the case, the observed fact is that, with the exception of Sgr and Leo I, the brightest satellites have velocity vectors within the VPOS. The models based on dark matter would have to account for why the fainter satellites are in the VPOS today while they would have motions that take them out of the VPOS on a time scale of $\Delta \tau \approx$ thickness/velocity dispersion $\approx 50 \mathrm{kpc} /$ $300 \mathrm{pc} / \mathrm{Myr}=167 \mathrm{Myr}$, which is significantly shorter than the age of the system and than the orbital time scale.

\subsection{Prediction: frequency of anisotropic dwarf satellite systems}

In Sec. 5.1.1 it has been found that the incidence of anisotropic satellite systems around host galaxies that have been well studied appears to be typical. In fact, nine such systems are already known, and in the Local Group, where the best 3D data with kinematical information is available, disk-of-satellites are the rule. This suggests that most satellite galaxies may be TDGs. This leads to the following prediction:

If most dwarf satellite galaxies are TDGs then anisotropic and rotating flattened satellite populations should be common, as long as encounters between major galaxies are typically less frequent than twice per Hubble time (Sec. 5.1).

Alternatively the known host galaxies with correlated satellite populations are exceptions.

But this is unlikely because, as emphasized by Chiboucas et al. (2013, [188]), in every case for which data exist so far, anisotropic and flattened satellite populations have been found (see Sec. 5.1.1). 


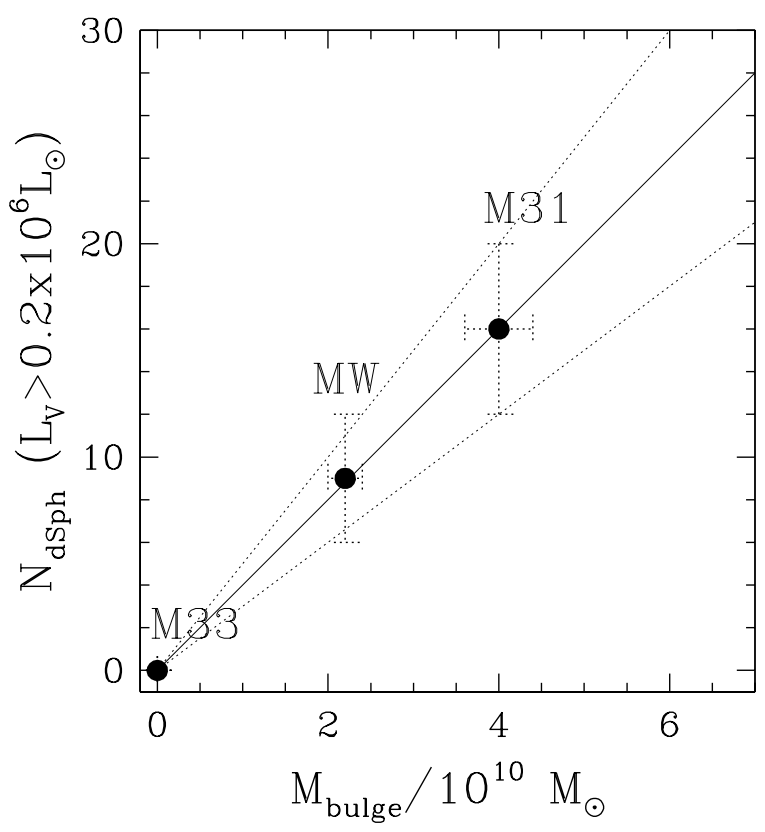

Fig. 11. The number of $\mathrm{dSph}$ and $\mathrm{dE}$ satellite galaxies, $N_{\text {sat }}$, each more luminous than $0.2 \times 10^{6} L_{\odot}$, is plotted versus the bulge mass, $M_{\text {bulge }}$ of the host galaxy. Only satellites within a distance of $270 \mathrm{kpc}$ of the MW and M 31 are used. The solid line is $N_{\text {sat }}=(4.03 \pm 0.04) \times M_{\text {bulge }} /\left(10^{10} M_{\odot}\right)$ (eq. 17 in [62]). See [62] for more details. Figure reprinted from [62]. Reproduced with permission from Astronomy \& Astrophysics, (c) ESO.

\subsection{Prediction: satellite galaxies and galactic bulges}

If the majority of dwarf satellite galaxies are TDGs, then the formation of satellite systems is associated with galaxygalaxy encounters. The formation of galactic bulges is also associated with galaxy-galaxy encounters because the torques in such encounters induce radial gas motions which can buildup a central spheroidal component on a time scale of 100 s of Myr. Thus, a correlation between the mass of the bulge and the number of satellite galaxies is expected in this scenario. In the Local Group such a correlation exists (Fig. 11). It is noteworthy that the MW has a bulge which appears to have formed within less than a Gyr [295, 296] about 10 Gyr ago and also before the thin disk of the MW. Together with the existence of an older thick disk this may be the smoking gun of a major encounter the young MW may have had about 9 to $11 \mathrm{Gyr}$ ago. This is a viable solution to the origin of the Local Group [181]. Note that the bulge of the MW may be a pseudo bulge formed from the early pre-thick disk structure as a result of a bar instability induced by the encounter.

The following prediction emerges [62]:

If most dwarf satellite galaxies are TDGs then their number around a host galaxy should correlate positively with the mass of the bulge of the host galaxy.

Alternatively, in the SMoC not the bulge mass but the circular velocity should correlate with the number of satellite galaxies because heavier dark matter halos have a larger number of subhalos within them [138].
This is unlikely to be the case because heavier dark matter halos are more likely to have had major mergers and are thus less likely to have major disk galaxies without classical bulges. But the majority of disk galaxies do not have a classical bulge $[148,149]$, while $94 \%$ of isolated disk galaxies which typically must also have grown via mergers if the $\mathrm{SMoC}$ is to be valid do not appear to have classical bulges [150].

\subsection{Prediction: Hickson compact groups}

Given that the existence of exotic dark matter particles appears to be inconsistent with the data, it follows that dynamical friction would not be a significant process for the evolution of galaxy pairs or groups of galaxies (Sec. 4.2). This allows to be tested by the following experiment suggested by $[5,62,332]$ :

Hickson compact groups of galaxies should not merge within a crossing time (see also the M81 case in Sec. 4.2.3). Thus, a survey of such groups about $1 \mathrm{Gyr}$ in the past, i.e. at a redshift of about 0.1 , ought to show about the same abundance of such groups if there is no dark matter.

Alternatively, Hickson compact groups of galaxies should merge within a crossing time (see also the M81 case in Sec. 4.2.3). A survey of such groups about $1 \mathrm{Gyr}$ in the past ought to show many more such groups if the SMoC were true. The remnants of merged Hickson compact groups should be evident as elliptical galaxies with significant young stellar populations. This would require Hickson compact groups to constantly reform from the field population of galaxies.

However, no prediction to this effect exists, and this seems already to be ruled out by the observation that the population of early-type galaxies does not evolve since the last 6 Gyr [152]. The observational constraints provided by [333] do not appear to support the notion that Hickson compact groups merge. Indeed, [332] write "we can conclude that SCGs ${ }^{13}$ are mostly gravitationally bound structures with a longer lifetime than that predicted by current numerical simulations."

As a cautionary note: there is evidence for young and intermediate-age stellar populations in some elliptical galaxies (e.g. [123]), and this is often interpreted to be due to mergers. While some mergers are likely to occur, due to Chandrasekhar dynamical friction on the extended stellar components and the ejection of orbital angular momentum and binding energy with massive tidal tails, mergers are much less frequent in Milgromian dynamics due to the absence of dynamical friction on the expansive dark matter halos. This have been studied by Tiret \& Combes (2008, [239]). The observed populations of young or intermediate-age stars in some E galaxies may also be formed when a pre-existing E galaxy interacts with a gasrich disk galaxy without merging. Encounters involving a wet galaxy can lead to one of the gaseous tidal arms being partially accreted where it can form a new generation of stars in the other galaxy. That tidal accretion is likely the dominant physical mechanism for making polar ring galaxies has been shown by Bournaud \& Combes (2003, [146]; see also [93,334]).

\footnotetext{
${ }^{1} \mathrm{SCG}$, southern compact group.
} 


\subsection{Prediction: TDGs and the BTFR}

If SID/Milgromian dynamics is the correct effective description of galaxies, then a trivial prediction follows:

Virialised TDGs must lie on the BTFR (Fig. 5), subject to the EFE.

Alternatively, if the arguments presented in this contribution are incorrect and if the SMoC were valid, then virialised isolated TDGs cannot lie on the BTFR but must be off set from it towards significantly smaller velocities.

\subsection{Prediction: sizes of satellite galaxies and galaxies in clusters}

The exposition of SID in Sec. 6 led to the possible existence of the "external field effect". If this effect is true then the following prediction ensues:

Elliptical galaxies in galaxy clusters and spheroidal satellite dwarf galaxies should be physically larger than their isolated counterparts due to the external field effect (Sec. 6.2).

Alternatively, if dwarf galaxies are in their own dark matter halos then the size of their baryonic component is typically unaffected through galactic tides and through the stripping of their dark matter halos even after the tidal stripping of over $99 \%$ of the initial dark halo mass [335].

\subsection{Prediction: number of massive stars in dwarf galaxies}

The rate with which galaxies accrete baryons can be inferred from the rate with which they are forming stars. The SFR is obtained from observations but depends on assuming whether the galaxy-wide IMF is an invariant stochastically sampled probability density distribution function, or whether it is a computable systematically varying IGIMF (Sec. 7). The various possibilities can be distinguished with the following star-count test based on the IGIMF prediction:

If the IGIMF theory is correct then late-type dwarf galaxies with $\mathrm{SFR}<10^{-2} M_{\odot} \mathrm{yr}^{-1}$ have on average a significantly smaller number of $\mathrm{O}$ stars than expected for an invariant stochastically sampled galaxy-wide IMF [281].

\section{Conclusions}

Based on assuming Einstein's field equations are valid in an extrapolation by many orders of magnitude in acceleration and spatial scale to galaxies and beyond, significant departures of observed motions from the expectations are found. The additional hypothesis that this indicates the presence of exotic dark matter particles is currently very popular and forms the basis of the standard model of cosmology. This SMoC is held by many to be an excellent account of the Universe, although many tensions between data and model have been and are being reported. Applying the dual dwarf galaxy theorem to the SMoC suggests it to be falsified: the available observational data do not allow a distinction to be found between the dynamical properties of primordial dwarf galaxies and tidal dwarf galaxies, although they must differ if the SMoC is true. At the

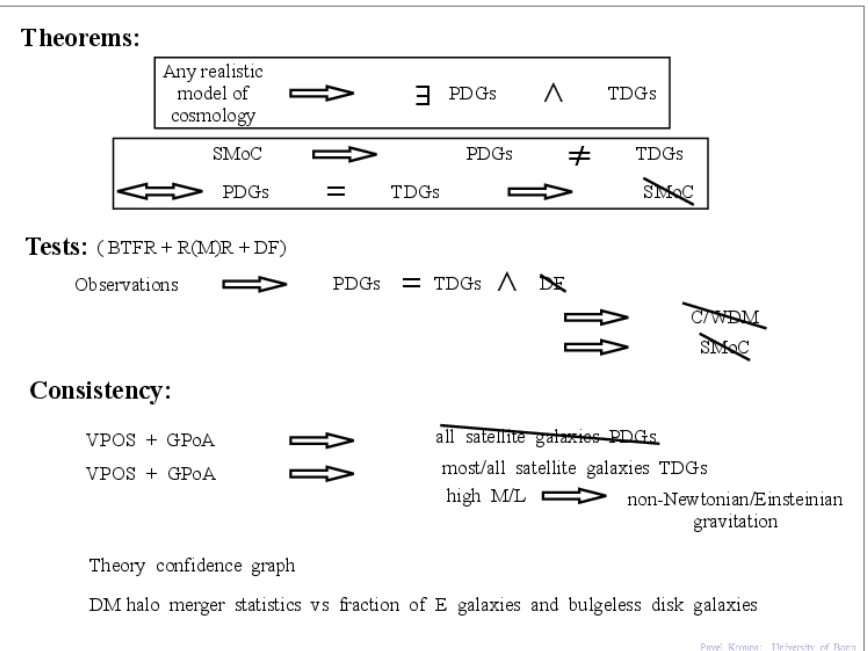

Fig. 12. Structure of the falsification of dynamically relevant cold or warm dark matter. The theorems are discussed in Sec. 3.3 and 4.1. The tests using the baryonic Tully Fisher relation (BTFR), the radius-mass relation $(\mathrm{R}(\mathrm{M}) \mathrm{R})$ of pressure supported dwarf galaxies and using dynamical friction (DF) are performed in Secs. 4.1.1, 4.1.2 and 4.2, respectively. The consistency checks based on the vast polar structure or the Milky Way (VPOS) and on the great plane of Andromeda (GPoA) are in Sec. 5.1.1. The theory confidence graph is discussed in Sec. 5.2 and the evidence for a lack of mergers in the observed galaxy population is covered in Sec. 4.2.4. Other acronyms: PDGs=primordial dwarf galaxies (Sec. 3.1) TDGs=tidal dwarf galaxies (Sec. 3.2), $\mathrm{C} / \mathrm{WDM}=$ cold or warm dark matter.

same time the arrangements of satellite dwarf galaxies in the VPOS around the MW and in the GPoA/VTDS around Andromeda and other anisotropic dwarf galaxy distributions are strongly suggestive of them being TDGs; the predicted large numbers of independently or group accreted, DM dominated spheroidally distributed dwarfs are most inconspicuously absent. Confident evidence for dynamical friction is found neither in interacting galaxies nor in the motions of satellite galaxies. The overall galaxy population is best explained with dynamical friction being suppressed. The most notorios case is the galaxy group of M81 (Sec. 4.2.3). Two independent groups have been unable to find solutions for the matter distribution within the M81 system as a result of the interactions between group members, assuming the SMoC to be valid. But solutions do exist for the case without dynamical friction. That the SMoC may not be the correct description of the Universe finds consistency in the documented history of many failures of the SMoC in accounting for observational data, as can be visualized with the theory-confidence graph (Sec. 5.2). None of these failures has been solved to-date. The structure of the exotic-dark-matter-falsification argument is depicted in compact form in Fig. 12.

The conclusion from these arguments based only on the available astronomical data, namely that exotic dark matter particles that are dynamically relevant on galaxy scales do not exist, would have deep consequences for current physics and uncomfortable sociological implications, but it is the reality that needs to be faced. This astronomical falsification of the cosmological concept of dark matter is echoed in the present-day 
null-evidence for physics beyond the SMoPP, despite searches at the highest available energies. The community had hoped to explain a number of issues in theoretical physics with physics beyond the SMoPP, but the null-results on evidence for such physics such as for supersymmetry is now putting theoretical approaches under crisis [42].

Thus, neither is there direct evidence for the existence of exotic dark matter particles, nor has experimental evidence for extensions of the SMoPP, which accommodate possible candidates for dark matter particles, been found.

As pointed out by Milgrom [216], gravitation on the scale of galaxies and beyond follows a very simple law which is referred to as scale-invariant dynamics. This SID leads to a number of interesting consequences, such as the baryonic Tully Fisher relation and possibly physically larger galaxies when they are subject to an external field from a nearby host. The tight correlation of observational data on the BTFR, which cannot be understood in terms of dark matter in the SMoC because the circular velocity of the dark matter halo is not well correlated with the baryonic galaxy within it [71], is a necessary consequence of this law of nature which all galaxies must abide by. The originally voiced need for a dark matter component to ensure disk stability by Ostriker \& Peebles (1973, [24]) and the requirement that this component be fine tuned with the baryonic one (Bahcall \& Casertano 1985, [25]) are both immediately and naturally solved by SID/Milgromian dynamics. That TDGs have been found to lie near the BTFR finds a trivial explanation in terms of SID. In SID, and for the same environmental conditions, primordial dwarf galaxies and virialised TDGs must look alike dynamically and morphologically, because they are required to follow this law of nature. Also, in SID pressure supported primordial dwarf galaxies and old TDGs must look alike, since there is no physical distinction between them apart from their epoch of formation. In SID there is therefore no tension between the dual dwarf galaxy theorem and observational data. In SID galaxies do not merge readily when they interact, because dynamical friction on dark matter halos does not occur. The present-day understanding of the dynamical behavior and of the evolution of galaxies needs to be revised because the dynamics of exotic dark matter particles do not play a role. Returning to the Gedankenexperiment "Challenge for Theory" in Sec. 2, it is by now clear that the rotation curve of the galaxy cannot be predicted within the SMoC, but it has a unique prediction in SID.

This revision is associated with a more thorough understanding of stellar populations in galaxies and the star-formationrate dependent IGIMF, which is leading to the insight that the chemo-dynamical evolution of galaxies may be more self-regulated and thus simpler than appreciated hitherto. In a major study of the evolution of the main-sequence of galaxies over cosmic time, Speagle et al. (2014. [151]) re-emphasize the major tension between the theoretical hierarchical-buildup of stellar mass in the SMoC in comparison with the observed rather simple self-similar evolution of galaxies, confirming the conclusions reached by Disney et al. (2008, [61]). Galaxy evolution appears to be fundamentally self-regulated and not a stochastic processes, such that dynamical-friction-driven mergers play a small if not an insignificant role. Cold or warm dark matter halos therefore do not appear to exist. Star-formation appears to be self-regulated, even on the smallest sub-pc scales, and follows well defined laws such that composite stellar populations, such as what galaxies are, become computable and predictable over cosmic time via the IGIMF theory and related concepts (e.g. dynamical population synthesis of binary star populations). Gauging the few parameters that enter the IGIMF theory using local observationally well resolved star formation, it follows that the galaxy-wide IMF (the IGIMF) changes systematically from top-light to top-heavy with increasing galaxy-wide star-formation rate. Late-type dwarf galaxies thus have a deficit of massive stars when compared to an assumed invariant fully sampled IMF while massive late-type galaxies have a top-heavy IMF. It emerges that there is no mystery in how the IMF is to know what type of galaxy it is forming in. Rather, the galaxy-wide IMF, i.e. the IGIMF, follows naturally from the simple addition of all star-forming events that are occurring in a galaxy which are distributed according to distribution functions that are subject to physical constraints, the most important one being the amount of mass being converted into the stellar masses of embedded clusters over about ten Myr timescales. The star-formation behavior of late type galaxies (the vastly dominant type of galaxy) yields the galaxy main sequence. It is consistent with the IGIMF theory, confirming the self-regulated evolution of galaxies rather than the haphazard processes associated with the SMoC. This advanced concept of galaxy evolution, which does not have an underlying merger tree [73], is shown schematically in Fig. 13.

The transition of SID to the Newtonian regime around gravitating masses may be derivable from the physical processes of the vacuum, as suggested by Milgrom (1999, [218], see Sec. 6.3 and 8). The complete description in the classical nonrelativistic regime, SID plus Newtonian dynamics, is referred to here as Milgromian dynamics. This framework accounts well for the observed internal properties of the satellite galaxies of Andromeda and the MW (e.g. [330,336]). It naturally leads to the kinematical properties of polar ring galaxies [337], and the anisotropic distribution of satellite galaxies can also be accounted for well in Milgromian dynamics [217]. Furthermore, the hitherto not expected highly symmetrical structure of the Local Group [91] may be a consequence of an early encounter between the MW and Andromeda [181], which is not possible in the SMoC because the two galaxies would have merged due to dynamical friction on their dark matter halos. Interpreting Milgromian dynamics as Einsteinian dynamics in the classical regime plus quantum-mechanical effects from the vacuum which become effective in the weak gravitational regime, a conservative cosmological model emerges which appears to be broadly consistent with available observational data on large and small scales (Sec. 8). Generalizations of Einstein's field equation (Sec. 2) may explicitly incorporate the physics of the vacuum, or they may have Milgromian gravitation as the correct classical limit.

The conclusion that exotic dark matter does not exist seems to be inescapable, if the data used for the tests remain valid and/or are verified, and thus new avenues for understanding gravitation in the weak field (acceleration $<a_{0} \approx 3.8 \mathrm{pc} /-$ $\mathrm{Myr}^{2}$ ) regime are encouraged. SID appears to be the correct effective description of the observed dynamics in this regime. An excellent in-depth review of various approaches is provided by [11]. Some ideas can be found for example in [338, 339] 


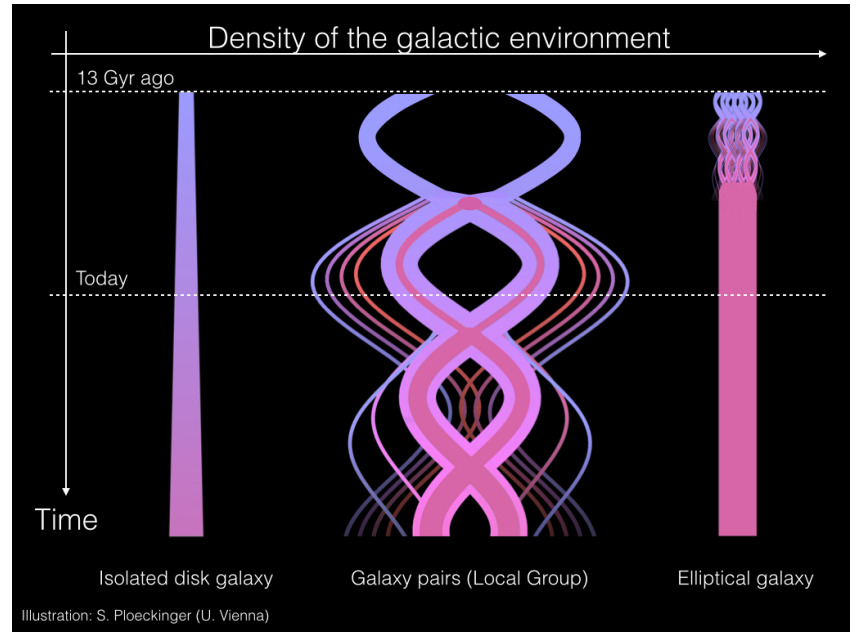

Fig. 13. Cartoon of galaxy evolution in SID in a universe without cold or warm dark matter (not to scale). The vast majority of galaxies with baryonic mass $M>10^{10} M_{\odot}$ are late-type since about 6 Gyr ago (about $97 \%$, [152], fig. 4.14 in [153]) and mergers do not play a significant role due to the absence of dynamical friction [238,239]. The left vertical blue track represents a growing largely non-interacting "island-universe" galaxy $[61,151,166]$. It reddens with time as the old stellar population builds up. It does not attain a classical bulge, but may appear as a chain galaxy $[254,255]$ if it experiences a disk-wide star-burst about $11 \mathrm{Gyr}$ ago, therewith building up a thick disk [256]. The central two tracks depict a Local Group with the motion of the MW and Andromeda around each other once they decouple from the cosmic expansion [181]. They spawn a new generation of TDGs at each encounter. These TDGs typically evolve to the present-day ( $t=$ today) faint red $\mathrm{dSph}$ and UFD satellite galaxies with bolometric luminosities $<10^{8} L_{\odot}$ but remain dIrr galaxies at larger distances from their hosts [101]. After each encounter the two galaxies obtain an increased bulge. A bulge-mass-satellite-number correlation arises (Fig. 11). The two major galaxies redden with time as the isolated galaxy on the left. This model of the Local Group would have looked as a peculiar galaxy about $11 \mathrm{Gyr}$ ago when the early MW and Andromeda interacted. A minority of galaxies with $M>10^{10} M_{\odot}$ (about $3 \%$, [152], fig. 4.14 in [153]) form in dense environments very early on through rapid accretion and gas-dominated mergers to become elliptical galaxies which evolve passively without significant later evolution. This had been deduced already by Matteucci (1994, [259]) and is depicted in the right-most track. These may have satellite galaxies which formed as TDGs during the early gaseous mergers. The observed size-evolution of these galaxies may be related to the external field effect in SID, but this has not been studied to date. Downsizing is accounted for in this cartoon. This cartoon supersedes the cosmological merger tree and fig. 9 in [62]. This figure was kindly provided by S. Plöckinger on an approach for understanding exotic dark matter and dark energy in terms of gravitational polarisation leading to SMoCproperties on large scales while recovering the correct SIDbehavior on galactic scales, in [340] on mirror dark matter, in [341] on modified gravity, in [342,343] on an approach with massive gravitons, in [171] on interpreting gravitation as an emergent property through a holographic scenario, and in [344-346] on a nonlocal generalization of Einstein's theory of gravitation in which a Yukawa-type repulsion appears and which reproduces galactic rotation curves and galaxy clusters without exotic dark matter. [20] gives an independent review and account of the issues covered here with a brief discussion of various approaches to non-standard models such as $f(R)$ gravity. Other independent reviews of related issues can be found in [58, 60, 64].

As a final note, the scientific community has put an incredible amount of effort to support and develop technologies to search for exotic dark matter and to perform computer simulations within the SMoC. There are nearly countless major research groups and centers world wide performing such simulations of the Universe. Attempts at establishing funding for even a small independent group in which numerical tools would be developed to perform similar simulations within the SID context have been rejected, on multiple occasions. In Bonn the POR code is being developed (Lüghausen, Famaey \& Kroupa 2014, [237]), on a small budget and thanks to local support from the university's rectorate. This code will allow simulations of galaxy formation and evolution within a Milgromian universe and is to become publicly available, but the future of this and similar projects is unclear. Cold or warm dark matter has not been found to this date, and if it does not exist, as this contribution argues based on astronomical data, then none of the simulations within the $\mathrm{SMoC}$ would represent reality. In a more realistic cosmological model the $\mathrm{CMB}$ may require a new interpretation [326] and, independently of this, distanceredshift-age relations may be different to those in the SMoC:

THE FUTURE OF COSMOLOGY: It follows that, without exotic cold or warm dark matter and with a systematically varying IGIMF with SFR, all observational quantities that are derived at present, such as star-formation rate densities, distances and ages from redshifts, and galaxy masses, are likely to require possibly major revision.

\section{Acknowledgements}

I thank the staff at the Institute for Astrophysics at the University of Vienna, and especially G. Hensler, for the hospitality during my guest professorship in March and April 2014. Much of this text was written in Vienna. M. Pawlowski, S. Plöckinger, S. Recchi, X. Wu and I. Stewart are thanked for helpful comments which improved this contribution. Benoit Famaey is thanked especially for providing me with important insights into the fundamental aspects of Milgromian dynamics. I also thank the staff at the Institute for Advanced Study (IAS) in Princeton for hosting me as a Visitor in April 2014, the place where Milgrom discovered his dynamics in the 1980s. Sec. 8 is a result of the discussions at the IAS. At the University of Bonn I would like to extend my gratitude to the Rektor, Jürgen 
Fohrmann, the Dean, Ulf Meissner, and Jürgen Kerp and Cristiano Porciani for playing helpful and decisive roles during the past 12 months.

\section{References}

1. W.-M. Yao, C. Amsler, D. Asner, R. M. Barnett, J. Beringer, P. R. Burchat, C. D. Carone, C. Caso, O. Dahl, G. D’Ambrosio, A. De Gouvea, M. Doser, S. Eidelman, J. L. Feng, T. Gherghetta, M. Goodman, C. Grab, D. E. Groom, A. Gurtu, K. Hagiwara, K. G. Hayes, J. J. Hernández-Rey, K. Hikasa, H. Jawahery, C. Kolda, Y. Kwon, M. L. Mangano, A. V. Manohar, A. Masoni, R. Miquel, K. Mönig, H. Murayama, K. Nakamura, S. Navas, K. A. Olive, L. Pape, C. Patrignani, A. Piepke, G. Punzi, G. Raffelt, J. G. Smith, M. Tanabashi, J. Terning, N. A. Törnqvist, T. G. sTrippe, P. Vogel, T. Watari, C. G. Wohl, R. L. Workman, P. A. Zyla, B. Armstrong, G. Harper, V. S. Lugovsky, P. Schaffner, M. Artuso, K. S. Babu, H. R. Band, E. Barberio, M. Battaglia, H. Bichsel, O. Biebel, P. Bloch, E. Blucher, R. N. Cahn, D. Casper, A. Cattai, A. Ceccucci, D. Chakraborty, R. S. Chivukula, G. Cowan, T. Damour, T. DeGrand, K. Desler, M. A. Dobbs, M. Drees, A. Edwards, D. A. Edwards, V. D. Elvira, J. Erler, V. V. Ezhela, W. Fetscher, B. D. Fields, B. Foster, D. Froidevaux, T. K. Gaisser, L. Garren, H.-J. Gerber, G. Gerbier, L. Gibbons, F. J. Gilman, G. F. Giudice, A. V. Gritsan, M. Grünewald, H. E. Haber, C. Hagmann, I. Hinchliffe, A. Höcker, P. Igo-Kemenes, J. D. JAckson, K. F. Johnson, D. Karlen, B. Kayser, D. Kirkby, S. R. Klein, K. Kleinknecht, I. G. Knowles, R. V. Kowalewski, P. Kreitz, B. Kursche, Y. V. Kuyanov, O. Lahav, P. Langacker, A. Liddle, Z. Ligeti, T. M. Liss, L. Littenberg, J. C. Liu, K. S. Lugovsky, s. B. Lugovsky, T. Mannel, D. M. Manley, W. J. Marciano, A. D. Martin, D. Milstead, M. Narain, P. Nason, Y. Nir, J. A. Peacock, S. A. Prell, A. Quadt, S. Raby, B. N. Ratcliff, E. A. Razuvaev, B. Renk, P. Richardson, S. Roesler, G. Rolandi, M. T. Ronan, L. J. Rosenberg, C. T. Sachrajda, Y. Sakai, S. Sarkar, M. Schmitt, O. Schneider, D. Scott, T. Sjöstrand, G. F. Smoot, P. Sokolsky, S. Spanier, H. Spieler, A. Stahl, T. Stanev, R. E. Streitmatter, T. Sumiyoshi, N. P. Tkachenko, G. H. Trilling, G. Valencia, K. van Bibber, M. G. Vincter, D. R. Ward, B. R. Webber, J. D. Wells, M. Whalley, L. Wolfenstsein, J. Womersley, C. L. Woody, A. Yamamoto, O. V. Zenin, J. Zhang, and R.-Y. Zhu. Review of Particle Physics. Journal of Physics G Nuclear Physics, 33:1-1232, July 2006.

2. A. Riotto. Possible cosmological explanation of why supersymmetry is hiding at the LHC. Phys. Rev. D, 86(12):125038, December 2012.

3. N. Shaham and T. Piran. Implications of the Penetration Depth of Ultrahigh-Energy Cosmic Rays on Physics at $100 \mathrm{TeV}$. Physical Review Letters, 110(2):021101, January 2013.

4. R. Pohl, R. Gilman, G. A. Miller, and K. Pachucki. Muonic Hydrogen and the Proton Radius Puzzle. Annual Review of Nuclear and Particle Science, 63:175-204, October 2013.

5. P. Kroupa. The Dark Matter Crisis: Falsification of the Current Standard Model of Cosmology. PASA, 29:395-433, June 2012.

6. R. A. Ibata, G. F. Lewis, A. R. Conn, M. J. Irwin, A. W. McConnachie, S. C. Chapman, M. L. Collins, M. Fardal, A. M. N. Ferguson, N. G. Ibata, A. D. Mackey, N. F. Martin, J. Navarro, R. M. Rich, D. Valls-Gabaud, and L. M. Widrow. A vast, thin plane of corotating dwarf galaxies orbiting the Andromeda galaxy. Nature, 493:62-65, January 2013.

7. A. R. Conn, G. F. Lewis, R. A. Ibata, Q. A. Parker, D. B. Zucker, A. W. McConnachie, N. F. Martin, D. Valls-Gabaud, N. Tanvir, M. J. Irwin, A. M. N. Ferguson, and S. C. Chapman. The Threedimensional Structure of the M31 Satellite System; Strong Evidence for an Inhomogeneous Distribution of Satellites. ApJ, 766:120, April 2013.

8. M. S. Pawlowski, J. Pflamm-Altenburg, and P. Kroupa. The VPOS: a vast polar structure of satellite galaxies, globular clusters and streams around the Milky Way. MNRAS, 423:1109_ 1126, June 2012.

9. M. S. Pawlowski, B. Famaey, H. Jerjen, D. Merritt, P. Kroupa, J. Dabringhausen, F. Lüghausen, D. A. Forbes, G. Hensler, F. Hammer, M. Puech, S. Fouquet, H. Flores, and Y. Yang. Coorbiting satellite galaxy structures are still in conflict with the distribution of primordial dwarf galaxies. MNRAS, 442:23622380, August 2014.

10. M. Milgrom. A modification of the Newtonian dynamics as a possible alternative to the hidden mass hypothesis. ApJ, 270:365-370, July 1983.

11. B. Famaey and S. S. McGaugh. Modified Newtonian Dynamics (MOND): Observational Phenomenology and Relativistic Extensions. Living Reviews in Relativity, 15:10, September 2012.

12. P. Di Bari, A. Masiero, and R. Mohapatra. Focus on the origin of matter. New Journal of Physics, 15(3):035030, March 2013.

13. R. A. Alpher, H. Bethe, and G. Gamow. The origin of chemical elements. Physical Review, 73:803-804, April 1948.

14. S. Burles, K. M. Nollett, and M. S. Turner. Big Bang Nucleosynthesis Predictions for Precision Cosmology. ApJL, 552:L1-L5, May 2001.

15. A. Coc, J.-P. Uzan, and E. Vangioni. Standard big bang nucleosynthesis and primordial CNO Abundances after Planck. ArXiv e-prints, March 2014.

16. BICEP2 Collaboration, P. A. R Ade, R. W. Aikin, D. Barkats, S. J. Benton, C. A. Bischoff, J. J. Bock, J. A. Brevik, I. Buder, E. Bullock, C. D. Dowell, L. Duband, J. P. Filippini, S. Fliescher, S. R. Golwala, M. Halpern, M. Hasselfield, S. R. Hildebrandt, G. C. Hilton, V. V. Hristov, K. D. Irwin, K. S. Karkare, J. P. Kaufman, B. G. Keating, S. A. Kernasovskiy, J. M. Kovac, C. L. Kuo, E. M. Leitch, M. Lueker, P. Mason, C. B. Netterfield, H. T. Nguyen, R. O’Brient, R. W. Ogburn, IV, A. Orlando, C. Pryke, C. D. Reintsema, S. Richter, R. Schwarz, C. D. Sheehy, Z. K. Staniszewski, R. V. Sudiwala, G. P. Teply, J. E. Tolan, A. D. Turner, A. G. Vieregg, C. L. Wong, and K. W. Yoon. BICEP2 I: Detection Of B-mode Polarization at Degree Angular Scales. ArXiv e-prints, March 2014.

17. R. Flauger, J. C. Hill, and D. N. Spergel. Toward an Understanding of Foreground Emission in the BICEP2 Region. ArXiv e-prints, May 2014.

18. M. J. Mortonson and U. Seljak. A joint analysis of Planck and BICEP2 B modes including dust polarization uncertainty. ArXiv e-prints, May 2014.

19. J. Einasto. Dark Matter. Brazilian Journal of Physics, 43:369374, December 2013.

20. S. Trippe. The "Missing Mass Problem" in Astronomy and the Need for a Modified Law of Gravity. Zeitschrift Naturforschung Teil A, 69:173-187, March 2014. 
21. A. Del Popolo. Nonbaryonic Dark Matter in Cosmology. International Journal of Modern Physics D, 23:30005, March 2014.

22. V. C. Rubin and W. K. Ford, Jr. Rotation of the Andromeda Nebula from a Spectroscopic Survey of Emission Regions. ApJ, 159:379, February 1970.

23. A. Bosma. 21-cm line studies of spiral galaxies. I - Observations of the galaxies NGC 5033, 3198, 5055, 2841, and 7331. II - The distribution and kinematics of neutral hydrogen in spiral galaxies of various morphological types. AJ, 86:1791-1846, December 1981.

24. J. P. Ostriker and P. J. E. Peebles. A Numerical Study of the Stability of Flattened Galaxies: or, can Cold Galaxies Survive? ApJ, 186:467-480, December 1973.

25. J. N. Bahcall and S. Casertano. Some possible regularities in the missing mass problem. ApJL, 293:L7-L10, June 1985.

26. M. Kilic, A. Gianninas, and T. von Hippel. Moving Objects in the Hubble Ultra Deep Field. ApJ, 774:88, September 2013.

27. K. Griest, A. M. Cieplak, and M. J. Lehner. New Limits on Primordial Black Hole Dark Matter from an Analysis of Kepler Source Microlensing Data. Physical Review Letters, 111(18):181302, November 2013.

28. F. Combes and D. Pfenniger. Perspectives for detecting cold H_2 in outer galactic disks. A\&A, 327:453-466, November 1997.

29. L. Bergström. Dark matter evidence, particle physics candidates and detection methods. Annalen der Physik, 524:479-496, October 2012.

30. G. R. Blumenthal, S. M. Faber, J. R. Primack, and M. J. Rees. Formation of galaxies and large-scale structure with cold dark matter. Nature, 311:517-525, October 1984.

31. M. Davis, G. Efstathiou, C. S. Frenk, and S. D. M. White. The evolution of large-scale structure in a universe dominated by cold dark matter. ApJ, 292:371-394, May 1985.

32. A. G. Riess, A. V. Filippenko, P. Challis, A. Clocchiatti, A. Diercks, P. M. Garnavich, R. L. Gilliland, C. J. Hogan, S. Jha, R. P. Kirshner, B. Leibundgut, M. M. Phillips, D. Reiss, B. P. Schmidt, R. A. Schommer, R. C. Smith, J. Spyromilio, C. Stubbs, N. B. Suntzeff, and J. Tonry. Observational Evidence from Supernovae for an Accelerating Universe and a Cosmological Constant. AJ, 116:1009-1038, September 1998.

33. B. P. Schmidt, N. B. Suntzeff, M. M. Phillips, R. A. Schommer, A. Clocchiatti, R. P. Kirshner, P. Garnavich, P. Challis, B. Leibundgut, J. Spyromilio, A. G. Riess, A. V. Filippenko, M. Hamuy, R. C. Smith, C. Hogan, C. Stubbs, A. Diercks, D. Reiss, R. Gilliland, J. Tonry, J. Maza, A. Dressler, J. Walsh, and R. Ciardullo. The High-Z Supernova Search: Measuring Cosmic Deceleration and Global Curvature of the Universe Using Type IA Supernovae. ApJ, 507:46-63, November 1998.

34. S. Perlmutter, G. Aldering, G. Goldhaber, R. A. Knop, P. Nugent, P. G. Castro, S. Deustua, S. Fabbro, A. Goobar, D. E. Groom, I. M. Hook, A. G. Kim, M. Y. Kim, J. C. Lee, N. J. Nunes, R. Pain, C. R. Pennypacker, R. Quimby, C. Lidman, R. S. Ellis, M. Irwin, R. G. McMahon, P. Ruiz-Lapuente, N. Walton, B. Schaefer, B. J. Boyle, A. V. Filippenko, T. Matheson, A. S. Fruchter, N. Panagia, H. J. M. Newberg, W. J. Couch, and Supernova Cosmology Project. Measurements of Omega and Lambda from 42 High-Redshift Supernovae. ApJ, 517:565-586, June 1999.
35. J. A. Frieman, M. S. Turner, and D. Huterer. Dark Energy and the Accelerating Universe. ARAA, 46:385-432, September 2008.

36. M. J. Mortonson, D. H. Weinberg, and M. White. Dark Energy: A Short Review. ArXiv e-prints, December 2014.

37. P. J. E. Peebles. Dark Matter. ArXiv e-prints, May 2013.

38. Planck Collaboration, P. A. R. Ade, N. Aghanim, C. ArmitageCaplan, M. Arnaud, M. Ashdown, F. Atrio-Barandela, J. Aumont, C. Baccigalupi, A. J. Banday, and et al. Planck 2013 results. XVI. Cosmological parameters. ArXiv e-prints, March 2013.

39. Y. Baryshev. Conceptual Problems of the Standard Cosmological Model. In E. J. Lerner and J. B. Almeida, editors, First Crisis in Cosmology Conference, volume 822 of American Institute of Physics Conference Series, pages 23-33, March 2006.

40. J. A. Peacock. Cosmological Physics. January 1999.

41. LUX Collaboration, D. S. Akerib, H. M. Araujo, X. Bai, A. J. Bailey, J. Balajthy, S. Bedikian, E. Bernard, A. Bernstein, A. Bolozdynya, A. Bradley, D. Byram, S. B. Cahn, M. C. Carmona-Benitez, C. Chan, J. J. Chapman, A. A. Chiller, C. Chiller, K. Clark, T. Coffey, A. Currie, A. Curioni, S. Dazeley, L. de Viveiros, A. Dobi, J. Dobson, E. M. Dragowsky, E. Druszkiewicz, B. Edwards, C. H. Faham, S. Fiorucci, C. Flores, R. J. Gaitskell, V. M. Gehman, C. Ghag, K. R. Gibson, M. G. D. Gilchriese, C. Hall, M. Hanhardt, S. A. Hertel, M. Horn, D. Q. Huang, M. Ihm, R. G. Jacobsen, L. Kastens, K. Kazkaz, R. Knoche, S. Kyre, R. Lander, N. A. Larsen, C. Lee, D. S. Leonard, K. T. Lesko, A. Lindote, M. I. Lopes, A. Lyashenko, D. C. Malling, R. Mannino, D. N. McKinsey, D.-M. Mei, J. Mock, M. Moongweluwan, J. Morad, M. Morii, A. S. J. Murphy, C. Nehrkorn, H. Nelson, F. Neves, J. A. Nikkel, R. A. Ott, M. Pangilinan, P. D. Parker, E. K. Pease, K. Pech, P. Phelps, L. Reichhart, T. Shutt, C. Silva, W. Skulski, C. J. Sofka, V. N. Solovov, P. Sorensen, T. Stiegler, K. O‘Sullivan, T. J. Sumner, R. Svoboda, M. Sweany, M. Szydagis, D. Taylor, B. Tennyson, D. R. Tiedt, M. Tripathi, S. Uvarov, J. R. Verbus, N. Walsh, R. Webb, J. T. White, D. White, M. S. Witherell, M. Wlasenko, F. L. H. Wolfs, M. Woods, and C. Zhang. First results from the LUX dark matter experiment at the Sanford Underground Research Facility. ArXiv e-prints, October 2013.

42. M. Shifman. Reflections and Impressionistic Portrait at the Conference "Frontiers Beyond the Standard Model," FTPI, Oct. 2012. ArXiv e-prints, October 2012.

43. A. Diaferio. The evidence for unusual gravity from the largescale structure of the Universe. ArXiv e-prints, February 2008.

44. S. S. McGaugh. A Tale of Two Paradigms: the Mutual Incommensurability of LCDM and MOND. ArXiv e-prints, April 2014.

45. A. Einstein. Die Grundlage der allgemeinen Relativitätstheorie. Annalen der Physik, 354:769-822, 1916.

46. M. P. Hobson, G. P. Efstathiou, and A. N. Lasenby. General Relativity. February 2006.

47. J. D. Bekenstein. Relativistic gravitation theory for the modified Newtonian dynamics paradigm. Phys. Rev. D, 70(8):083509, October 2004.

48. J. D. Bekenstein. Tensor-vector-scalar-modified gravity: from small scale to cosmology. Royal Society of London Philosophical Transactions Series A, 369:5003-5017, December 2011. 
49. T. G. Zlosnik, P. G. Ferreira, and G. D. Starkman. Modifying gravity with the aether: An alternative to dark matter. Phys. Rev. D, 75(4):044017, February 2007.

50. M. Milgrom. Bimetric MOND gravity. Phys. Rev. D, 80(12):123536, December 2009.

51. C. Deffayet, G. Esposito-Farese, and R. P. Woodard. Field equations and cosmology for a class of nonlocal metric models of MOND. ArXiv e-prints, May 2014.

52. J. R. Primack. Cosmology: small-scale issues. New Journal of Physics, 11(10):105029, October 2009.

53. C. Scannapieco, M. Wadepuhl, O. H. Parry, J. F. Navarro, A. Jenkins, V. Springel, R. Teyssier, E. Carlson, H. M. P. Couchman, R. A. Crain, C. Dalla Vecchia, C. S. Frenk, C. Kobayashi, P. Monaco, G. Murante, T. Okamoto, T. Quinn, J. Schaye, G. S. Stinson, T. Theuns, J. Wadsley, S. D. M. White, and R. Woods. The Aquila comparison project: the effects of feedback and numerical methods on simulations of galaxy formation. MNRAS, 423:1726-1749, June 2012.

54. R. H. Sanders. Mass discrepancies in galaxies - Dark matter and alternatives. AAPR, 2:1-28, 1990.

55. S. S. McGaugh and W. J. G. de Blok. Testing the Dark Matter Hypothesis with Low Surface Brightness Galaxies and Other Evidence. ApJ, 499:41, May 1998.

56. M. J. Disney. The Case Against Cosmology. General Relativity and Gravitation, 32:1125-1134, June 2000.

57. F. Matteucci. What determines galactic evolution? Astrophysics and Space Science, 284:539-548, April 2003.

58. R. Scarpa. Modified Newtonian Dynamics, an Introductory Review. In E. J. Lerner and J. B. Almeida, editors, First Crisis in Cosmology Conference, volume 822 of American Institute of Physics Conference Series, pages 253-265, March 2006.

59. J. R. Primack. Precision Cosmology: Successes and Challenges. Nuclear Physics B Proceedings Supplements, 173:1-5, November 2007.

60. R. Sanders. Modified Gravity Without Dark Matter. In L. Papantonopoulos, editor, The Invisible Universe: Dark Matter and Dark Energy, volume 720 of Lecture Notes in Physics, Berlin Springer Verlag, page 375, 2007.

61. M. J. Disney, J. D. Romano, D. A. Garcia-Appadoo, A. A. West, J. J. Dalcanton, and L. Cortese. Galaxies appear simpler than expected. Nature, 455:1082-1084, October 2008.

62. P. Kroupa, B. Famaey, K. S. de Boer, J. Dabringhausen, M. S. Pawlowski, C. M. Boily, H. Jerjen, D. Forbes, G. Hensler, and M. Metz. Local-Group tests of dark-matter concordance cosmology . Towards a new paradigm for structure formation. A\&A, 523:A32, November 2010.

63. P. J. E. Peebles and A. Nusser. Nearby galaxies as pointers to a better theory of cosmic evolution. Nature, 465:565-569, June 2010.

64. X. Hernandez, A. Jimenez, and C. Allen. Gravitational anomalies signaling the breakdown of classical gravity. ArXiv e-prints, January 2014.

65. M. G. Walker and A. Loeb. Is the Universe Simpler than LCDM? ArXiv e-prints, January 2014.

66. R. H. Sanders. A dearth of dark matter in strong gravitational lenses. MNRAS, 439:1781-1786, February 2014.

67. H. J. de Vega, M. C. Falvella, and N. G. Sanchez. Highlights and Conclusions of the Chalonge 14th Paris Cosmology Colloquium 2010: 'The Standard Model of the Universe: Theory and Observations'. ArXiv e-prints, September 2010.
68. I. D. Karachentsev. Missing dark matter in the local universe. Astrophysical Bulletin, 67:123-134, April 2012.

69. R. C. Keenan, A. J. Barger, and L. L. Cowie. Evidence for a 300 Megaparsec Scale Under-density in the Local Galaxy Distribution. ApJ, 775:62, September 2013.

70. I. Ferrero, M. G. Abadi, J. F. Navarro, L. V. Sales, and S. Gurovich. The dark matter haloes of dwarf galaxies: a challenge for the $\Lambda$ cold dark matter paradigm? MNRAS, 425:28172823, October 2012.

71. Y. Lu, H. J. Mo, N. Katz, and M. D. Weinberg. Bayesian inference of galaxy formation from the K-band luminosity function of galaxies: tensions between theory and observation. MNRAS, 421:1779-1796, April 2012.

72. F. Shankar, S. Mei, M. Huertas-Company, J. Moreno, F. Fontanot, P. Monaco, M. Bernardi, A. Cattaneo, R. Sheth, R. Licitra, L. Delaye, and A. Raichoor. Environmental dependence of bulge-dominated galaxy sizes in hierarchical models of galaxy formation. Comparison with the local Universe. MNRAS, 439:3189-3212, February 2014.

73. C. Lacey and S. Cole. Merger rates in hierarchical models of galaxy formation. MNRAS, 262:627-649, June 1993.

74. K. R. Stewart, J. S. Bullock, R. H. Wechsler, A. H. Maller, and A. R. Zentner. Merger Histories of Galaxy Halos and Implications for Disk Survival. ApJ, 683:597-610, August 2008.

75. O. Fakhouri, C.-P. Ma, and M. Boylan-Kolchin. The merger rates and mass assembly histories of dark matter haloes in the two Millennium simulations. MNRAS, 406:2267-2278, August 2010.

76. A. Pontzen and F. Governato. Cold dark matter heats up. Nature, 506:171-178, February 2014.

77. P. Kroupa. The dynamical evolution of stellar superclusters. MNRAS, 300:200-204, October 1998.

78. D. A. Forbes and P. Kroupa. What Is a Galaxy? Cast Your Vote Here. PASA, 28:77-82, March 2011.

79. S. J. Aarseth. Gravitational N-Body Simulations. October 2003.

80. E. K. Grebel and J. S. Gallagher, III. The Impact of Reionization on the Stellar Populations of Nearby Dwarf Galaxies. ApJL, 610:L89-L92, August 2004.

81. A. Klypin, A. V. Kravtsov, O. Valenzuela, and F. Prada. Where Are the Missing Galactic Satellites? ApJ, 522:82-92, September 1999.

82. B. Moore, S. Ghigna, F. Governato, G. Lake, T. Quinn, J. Stadel, and P. Tozzi. Dark Matter Substructure within Galactic Halos. ApJL, 524:L19-L22, October 1999.

83. J. Diemand, M. Kuhlen, P. Madau, M. Zemp, B. Moore, D. Potter, and J. Stadel. Clumps and streams in the local dark matter distribution. Nature, 454:735-738, August 2008.

84. A. A. Klypin, S. Trujillo-Gomez, and J. Primack. Dark Matter Halos in the Standard Cosmological Model: Results from the Bolshoi Simulation. ApJ, 740:102, October 2011.

85. M. Metz, P. Kroupa, and H. Jerjen. The spatial distribution of the Milky Way and Andromeda satellite galaxies. MNRAS, 374:1125-1145, January 2007.

86. M. S. Pawlowski, P. Kroupa, G. Angus, K. S. de Boer, B. Famaey, and G. Hensler. Filamentary accretion cannot explain the orbital poles of the Milky Way satellites. MNRAS, 424:80-92, July 2012.

87. D. F. Watson, A. P. Hearin, A. A. Berlind, M. R. Becker, P. S. Behroozi, R. A. Skibba, R. Reyes, and A. R. Zentner. Predicting 
Galaxy Star Formation Rates via the Co-evolution of Galaxies and Halos. ArXiv e-prints, March 2014.

88. W. Wang, L. V. Sales, B. M. B. Henriques, and S. D. M. White. Satellite abundances around bright isolated galaxies II: radial distribution and environmental effects. ArXiv e-prints, March 2014.

89. R. A. Ibata, N. G. Ibata, G. F. Lewis, N. F. Martin, A. Conn, P. Elahi, V. Arias, and N. Fernando. A Thousand Shadows of Andromeda: Rotating Planes of Satellites in the Millennium-II Cosmological Simulation. ApJL, 784:L6, March 2014.

90. J. D. Simon and M. Geha. The Kinematics of the Ultra-faint Milky Way Satellites: Solving the Missing Satellite Problem. ApJ, 670:313-331, November 2007.

91. M. S. Pawlowski, P. Kroupa, and H. Jerjen. Dwarf galaxy planes: the discovery of symmetric structures in the Local Group. MNRAS, 435:1928-1957, November 2013.

92. M. S. Pawlowski and P. Kroupa. The rotationally stabilized VPOS and predicted proper motions of the Milky Way satellite galaxies. MNRAS, 435:2116-2131, November 2013.

93. M. S. Pawlowski, P. Kroupa, and K. S. de Boer. Making counterorbiting tidal debris. The origin of the Milky Way disc of satellites? A\&A, 532:A118, August 2011.

94. V. Belokurov, M. J. Irwin, S. E. Koposov, N. W. Evans, E. Gonzalez-Solares, N. Metcalfe, and T. Shanks. ATLAS lifts the Cup: Discovery of a New Milky Way satellite in Crater. ArXiv e-prints, March 2014.

95. B. P. M. Laevens, N. F. Martin, B. Sesar, E. J. Bernard, H.-W. Rix, C. T. Slater, E. F. Bell, A. M. N. Ferguson, E. F. Schlafly, W. S. Burgett, K. C. Chambers, L. Denneau, P. W. Draper, N. Kaiser, R.-P. Kudritzki, E. A. Magnier, N. Metcalfe, J. S. Morgan, P. A. Price, W. E. Sweeney, J. L. Tonry, R. J. Wainscoat, and C. Waters. A New Distant Milky Way Globular Cluster in the Pan-STARRS1 3 $\pi$ Survey. ApJL, 786:L3, May 2014.

96. M. S. Pawlowski and P. Kroupa. The Vast Polar Structure of the Milky Way Attains New Members. ApJ, 790:74, July 2014.

97. B. Moore, J. Diemand, P. Madau, M. Zemp, and J. Stadel. Globular clusters, satellite galaxies and stellar haloes from early dark matter peaks. MNRAS, 368:563-570, May 2006.

98. N. I. Libeskind, S. Cole, C. S. Frenk, T. Okamoto, and A. Jenkins. Satellite systems around galaxies in hydrodynamic simulations. MNRAS, 374:16-28, January 2007.

99. E. J. Tollerud, J. S. Bullock, L. E. Strigari, and B. Willman. Hundreds of Milky Way Satellites? Luminosity Bias in the Satellite Luminosity Function. ApJ, 688:277-289, November 2008.

100. V. Belokurov. Galactic Archaeology: The dwarfs that survived and perished. NAR, 57:100-121, September 2013.

101. D. A. Hunter, S. D. Hunsberger, and E. W. Roye. Identifying Old Tidal Dwarf Irregulars. ApJ, 542:137-142, October 2000.

102. F. Bournaud, P.-A. Duc, and E. Emsellem. High-resolution simulations of galaxy mergers: resolving globular cluster formation. MNRAS, 389:L8-L12, September 2008.

103. F. Menanteau, H. C. Ford, G. D. Illingworth, M. Sirianni, J. P. Blakeslee, G. R. Meurer, A. R. Martel, N. Benítez, M. Postman, M. Franx, D. R. Ardila, F. Bartko, R. J. Bouwens, T. J. Broadhurst, R. A. Brown, C. J. Burrows, E. S. Cheng, M. Clampin, N. J. G. Cross, P. D. Feldman, D. A. Golimowski, C. Gronwall, G. F. Hartig, L. Infante, R. A. Kimble, J. E. Krist, M. P. Lesser, G. K. Miley, P. Rosati, W. B. Sparks, H. D. Tran, Z. I. Tsvetanov, R. L. White, and W. Zheng. Internal Color Properties of Resolved Spheroids in the Deep Hubble Space Telescope Advanced
Camera for Surveys Field of UGC 10214. ApJ, 612:202-214, September 2004.

104. M. Wetzstein, T. Naab, and A. Burkert. Do dwarf galaxies form in tidal tails? MNRAS, 375:805-820, March 2007.

105. S. Fouquet, F. Hammer, Y. Yang, M. Puech, and H. Flores. Does the dwarf galaxy system of the Milky Way originate from Andromeda? MNRAS, 427:1769-1783, December 2012.

106. F. Hammer, Y. Yang, S. Fouquet, M. S. Pawlowski, P. Kroupa, M. Puech, H. Flores, and J. Wang. The vast thin plane of M31 corotating dwarfs: an additional fossil signature of the M31 merger and of its considerable impact in the whole Local Group. MNRAS, 431:3543-3549, June 2013.

107. S. Ploeckinger, G. Hensler, S. Recchi, N. Mitchell, and P. Kroupa. Chemo-dynamical evolution of tidal dwarf galaxies. I. Method and IMF dependence. MNRAS, 437:3980-3993, February 2014.

108. P.-A. Duc, S. Paudel, R. M. McDermid, J.-C. Cuillandre, P. Serra, F. Bournaud, M. Cappellari, and E. Emsellem. Identification of old tidal dwarfs near early-type galaxies from deep imaging and H I observations. MNRAS, 440:1458-1469, March 2014.

109. J. E. Barnes and L. Hernquist. Formation of dwarf galaxies in tidal tails. Nature, 360:715-717, December 1992.

110. B. G. Elmegreen, M. Kaufman, and M. Thomasson. An interaction model for the formation of dwarf galaxies and $10 \exp 8$ solar mass clouds in spiral disks. ApJ, 412:90-98, July 1993.

111. P. Kroupa. Dwarf spheroidal satellite galaxies without dark matter. NA, 2:139-164, July 1997.

112. F. Bournaud. Tidal Dwarf Galaxies and Missing Baryons. Advances in Astronomy, 2010, 2010.

113. S. Piatek and C. Pryor. The effect of galactic tides on the apparent mass-to-light ratios in dwarf spheroidal galaxies. $\mathrm{AJ}$, 109:1071-1085, March 1995.

114. R. S. Klessen and P. Kroupa. Dwarf Spheroidal Satellite Galaxies without Dark Matter: Results from Two Different Numerical Techniques. ApJ, 498:143, May 1998.

115. R. A. Casas, V. Arias, K. Peña Ramírez, and P. Kroupa. Dwarf spheroidal satellites of the Milky Way from dark matter free tidal dwarf galaxy progenitors: maps of orbits. MNRAS, 424:19411951, August 2012.

116. J. Dabringhausen and P. Kroupa. Dwarf elliptical galaxies as ancient tidal dwarf galaxies. MNRAS, 429:1858-1871, March 2013.

117. M. J. Disney and R. H. Lang. The galaxy ancestor problem. MNRAS, 426:1731-1749, November 2012.

118. T. Okazaki and Y. Taniguchi. Dwarf Galaxy Formation Induced by Galaxy Interactions. ApJ, 543:149-152, November 2000.

119. T. Lisker. Early-type dwarf galaxies in clusters: A mixed bag with various origins? Astronomische Nachrichten, 330:1043, December 2009.

120. D. A. Forbes, L. R. Spitler, A. W. Graham, C. Foster, G. K. T. Hau, and A. Benson. Bridging the gap between low- and highmass dwarf galaxies. MNRAS, 413:2665-2678, June 2011.

121. S. Recchi, C. Theis, P. Kroupa, and G. Hensler. The early evolution of tidal dwarf galaxies. A\&A, 470:L5-L8, July 2007.

122. F. Bournaud, P.-A. Duc, E. Brinks, M. Boquien, P. Amram, U. Lisenfeld, B. S. Koribalski, F. Walter, and V. Charmandaris. Missing Mass in Collisional Debris from Galaxies. Science, 316:1166-, May 2007. 
123. P.-A. Duc, J.-C. Cuillandre, P. Serra, L. Michel-Dansac, E. Ferriere, K. Alatalo, L. Blitz, M. Bois, F. Bournaud, M. Bureau, M. Cappellari, R. L. Davies, T. A. Davis, P. T. de Zeeuw, E. Emsellem, S. Khochfar, D. Krajnović, H. Kuntschner, P.-Y. Lablanche, R. M. McDermid, R. Morganti, T. Naab, T. Oosterloo, M. Sarzi, N. Scott, A.-M. Weijmans, and L. M. Young. The ATLAS $^{3 D}$ project - IX. The merger origin of a fast- and a slowrotating early-type galaxy revealed with deep optical imaging: first results. MNRAS, 417:863-881, October 2011.

124. P. S. Behroozi, R. H. Wechsler, and C. Conroy. The Average Star Formation Histories of Galaxies in Dark Matter Halos from $\mathrm{z}=$ 0-8. ApJ, 770:57, June 2013.

125. S. S. McGaugh. The Baryonic Tully-Fisher Relation of Gas-rich Galaxies as a Test of $\Lambda \mathrm{CDM}$ and MOND. AJ, 143:40, February 2012.

126. G. Gentile, B. Famaey, F. Combes, P. Kroupa, H. S. Zhao, and O. Tiret. Tidal dwarf galaxies as a test of fundamental physics. A\&A, 472:L25-L28, September 2007.

127. K. G. Begeman, A. H. Broeils, and R. H. Sanders. Extended rotation curves of spiral galaxies - Dark haloes and modified dynamics. MNRAS, 249:523-537, April 1991.

128. K. V. Johnston, S. R. Majewski, M. H. Siegel, I. N. Reid, and W. E. Kunkel. Constraining the History of the Sagittarius Dwarf Galaxy Using Observations of Its Tidal Debris. AJ, 118:17191726, October 1999.

129. R. Ibata, G. F. Lewis, M. Irwin, E. Totten, and T. Quinn. Great Circle Tidal Streams: Evidence for a Nearly Spherical Massive Dark Halo around the Milky Way. ApJ, 551:294-311, April 2001.

130. A. Helmi and S. D. M. White. Simple dynamical models of the Sagittarius dwarf galaxy. MNRAS, 323:529-536, May 2001.

131. M. Fellhauer, V. Belokurov, N. W. Evans, M. I. Wilkinson, D. B. Zucker, G. Gilmore, M. J. Irwin, D. M. Bramich, S. Vidrih, R. F. G. Wyse, T. C. Beers, and J. Brinkmann. The Origin of the Bifurcation in the Sagittarius Stream. ApJ, 651:167-173, November 2006.

132. D. R. Law and S. R. Majewski. The Sagittarius Dwarf Galaxy: A Model for Evolution in a Triaxial Milky Way Halo. ApJ, 714:229-254, May 2010.

133. M. Correnti, M. Bellazzini, R. A. Ibata, F. R. Ferraro, and A. Varghese. The Northern Wraps of the Sagittarius Stream as Traced by Red Clump Stars: Distances, Intrinsic Widths, and Stellar Densities. ApJ, 721:329-356, September 2010.

134. C. Vera-Ciro and A. Helmi. Constraints on the Shape of the Milky Way Dark Matter Halo from the Sagittarius Stream. ApJL, 773:L4, August 2013.

135. M. A. Gómez-Flechoso, R. Fux, and L. Martinet. Sagittarius, a dwarf spheroidal galaxy without dark matter? A\&A, 347:77-91, July 1999.

136. C. W. Purcell, J. S. Bullock, E. J. Tollerud, M. Rocha, and S. Chakrabarti. The Sagittarius impact as an architect of spirality and outer rings in the Milky Way. Nature, 477:301-303, September 2011.

137. J. Binney and S. Tremaine. Galactic dynamics. 1987.

138. T. Ishiyama, S. Rieder, J. Makino, S. Portegies Zwart, D. Groen, K. Nitadori, C. de Laat, S. McMillan, K. Hiraki, and S. Harfst. The Cosmogrid Simulation: Statistical Properties of Small Dark Matter Halos. ApJ, 767:146, April 2013.
139. H. Zhao. The Survival of the Sagittarius Dwarf Galaxy and the Flatness of the Rotation Curve of the Galaxy. ApJL, 500:L149, June 1998.

140. G. W. Angus, A. Diaferio, and P. Kroupa. Using dwarf satellite proper motions to determine their origin. MNRAS, 416:14011409, September 2011.

141. R. C. Thomson, S. Laine, and A. Turnbull. Towards an Interaction Model of M81, M82 and NGC 3077. In J. E. Barnes and D. B. Sanders, editors, Galaxy Interactions at Low and High Redshift, volume 186 of IAU Symposium, page 135, 1999.

142. M. S. Yun. Tidal Interactions in M81 Group. In J. E. Barnes and D. B. Sanders, editors, Galaxy Interactions at Low and High Redshift, volume 186 of IAU Symposium, page 81, 1999.

143. M. Thomasson and K. J. Donner. A Model of the Tidal Interaction Between M81 and NGC3077. A\&A, 272:153, May 1993.

144. J. E. Barnes. Dynamics of Galaxy Interactions. In R. C. Kennicutt, Jr., F. Schweizer, J. E. Barnes, D. Friedli, L. Martinet, and D. Pfenniger, editors, Saas-Fee Advanced Course 26: Galaxies: Interactions and Induced Star Formation, page 275, 1998.

145. G. C. Privon, J. E. Barnes, A. S. Evans, J. E. Hibbard, M. S. Yun, J. M. Mazzarella, L. Armus, and J. Surace. Dynamical Modeling of Galaxy Mergers Using Identikit. ApJ, 771:120, July 2013.

146. F. Bournaud and F. Combes. Formation of polar ring galaxies. A\&A, 401:817-833, April 2003.

147. A. Toomre. Mergers and Some Consequences. In B. M. Tinsley and R. B. G. Larson, D. Campbell, editors, Evolution of Galaxies and Stellar Populations, page 401, 1977.

148. T. Weinzirl, S. Jogee, S. Khochfar, A. Burkert, and J. Kormendy. Bulge $\mathrm{n}$ and B/T in High-Mass Galaxies: Constraints on the Origin of Bulges in Hierarchical Models. ApJ, 696:411-447, May 2009.

149. J. Kormendy, N. Drory, R. Bender, and M. E. Cornell. Bulgeless Giant Galaxies Challenge Our Picture of Galaxy Formation by Hierarchical Clustering. ApJ, 723:54-80, November 2010.

150. M. Fernández Lorenzo, J. Sulentic, L. Verdes-Montenegro, J. Blasco-Herrera, M. Argudo-Fernández, J. Garrido, P. Ramírez-Moreta, J. E. Ruiz, S. Sánchez-Expósito, and J. D. Santander-Vela. Are (pseudo)bulges in isolated galaxies actually primordial relics? ArXiv e-prints, May 2014.

151. J. S. Speagle, C. L. Steinhardt, P. L. Capak, and J. D. Silverman. A Highly Consistent Framework for the Evolution of the StarForming "Main Sequence" from z 0-6. ArXiv e-prints, May 2014.

152. R. Delgado-Serrano, F. Hammer, Y. B. Yang, M. Puech, H. Flores, and M. Rodrigues. How was the Hubble sequence $6 \mathrm{Gyr}$ ago? A\&A, 509:A78, January 2010.

153. J. Binney and M. Merrifield. Galactic Astronomy, Princeton University Press. 1998.

154. M. Puech, F. Hammer, P. F. Hopkins, E. Athanassoula, H. Flores, M. Rodrigues, J. L. Wang, and Y. B. Yang. Galaxy Disks Do Not Need to Survive in the $\Lambda$ CDM Paradigm: The Galaxy Merger Rate Out to $\mathrm{z}^{\sim} 1.5$ from Morpho-kinematic Data. ApJ, 753:128, July 2012.

155. T. Naab and J. P. Ostriker. Are Disk Galaxies the Progenitors of Giant Ellipticals? ApJ, 690:1452-1462, January 2009.

156. X. Wu and P. Kroupa. Galactic rotation curves, the baryonto-dark-halo-mass relation and space-time scale invariance. MNRAS, 446:330-344, January 2015.

157. T. J. Cox and A. Loeb. The collision between the Milky Way and Andromeda. MNRAS, 386:461-474, May 2008. 
158. I. R. Walker, J. C. Mihos, and L. Hernquist. Quantifying the Fragility of Galactic Disks in Minor Mergers. ApJ, 460:121, March 1996.

159. T. Naab and A. Burkert. Statistical Properties of Collisionless Equal- and Unequal-Mass Merger Remnants of Disk Galaxies. ApJ, 597:893-906, November 2003.

160. S. Kazantzidis, A. R. Zentner, A. V. Kravtsov, J. S. Bullock, and V. P. Debattista. Cold Dark Matter Substructure and Galactic Disks. II. Dynamical Effects of Hierarchical Satellite Accretion. ApJ, 700:1896-1920, August 2009.

161. O. Agertz, R. Teyssier, and B. Moore. The formation of disc galaxies in a $\Lambda$ CDM universe. MNRAS, 410:1391-1408, January 2011.

162. J. Guedes, S. Callegari, P. Madau, and L. Mayer. Forming Realistic Late-type Spirals in a $\Lambda$ CDM Universe: The Eris Simulation. ApJ, 742:76, December 2011.

163. M. Aumer, S. D. M. White, T. Naab, and C. Scannapieco. Towards a more realistic population of bright spiral galaxies in cosmological simulations. MNRAS, 434:3142-3164, October 2013.

164. F. Marinacci, R. Pakmor, and V. Springel. The formation of disc galaxies in high-resolution moving-mesh cosmological simulations. MNRAS, 437:1750-1775, January 2014.

165. S. S. McGaugh. The Mass Discrepancy-Acceleration Relation: Disk Mass and the Dark Matter Distribution. ApJ, 609:652-666, July 2004.

166. M. Samland and O. E. Gerhard. The formation of a disk galaxy within a growing dark halo. A\&A, 399:961-982, March 2003.

167. M. Vogelsberger, S. Genel, V. Springel, P. Torrey, D. Sijacki, D. Xu, G. F. Snyder, S. Bird, D. Nelson, and L. Hernquist. Properties of galaxies reproduced by a hydrodynamic simulation. ArXiv e-prints, May 2014.

168. M. Vogelsberger, S. Genel, V. Springel, P. Torrey, D. Sijacki, D. Xu, G. F. Snyder, D. Nelson, and L. Hernquist. Introducing the Illustris Project: Simulating the coevolution of dark and visible matter in the Universe. ArXiv e-prints, May 2014.

169. R. Roškar, R. Teyssier, O. Agertz, M. Wetzstein, and B. Moore. A Systematic Look at the Effects of Radiative Feedback on Disc Galaxy Formation. ArXiv e-prints, August 2014.

170. R. Lang. Dunkle Materie bleibt im Dunklen. Physik Journal, March, 13:18, 2014.

171. E. Verlinde. On the origin of gravity and the laws of Newton. Journal of High Energy Physics, 4:29, April 2011.

172. R. B. Tully, L. Rizzi, A. E. Dolphin, I. D. Karachentsev, V. E. Karachentseva, D. I. Makarov, L. Makarova, S. Sakai, and E. J. Shaya. Associations of Dwarf Galaxies. AJ, 132:729-748, August 2006.

173. M. Metz, P. Kroupa, C. Theis, G. Hensler, and H. Jerjen. Did the Milky Way Dwarf Satellites Enter The Halo as a Group? ApJ, 697:269-274, May 2009.

174. D. Lynden-Bell. Dwarf galaxies and globular clusters in high velocity hydrogen streams. MNRAS, 174:695-710, March 1976.

175. D. Lynden-Bell. The Fornax-Leo-Sculptor system. The Observatory, 102:202-208, October 1982.

176. W. E. Kunkel and S. Demers. The Magellanic Plane. In R. J. Dickens, J. E. Perry, F. G. Smith, and I. R. King, editors, The Galaxy and the Local Group, volume 182 of Royal Greenwich Observatory Bulletins, page 241, 1976.
177. P. Kroupa, C. Theis, and C. M. Boily. The great disk of MilkyWay satellites and cosmological sub-structures. A\&A, 431:517521, February 2005.

178. M. Metz and P. Kroupa. Dwarf spheroidal satellites: are they of tidal origin? MNRAS, 376:387-392, March 2007.

179. Y. Yang, F. Hammer, S. Fouquet, H. Flores, M. Puech, M. S. Pawlowski, and P. Kroupa. Reproducing properties of MW dSphs as descendants of DM-free TDGs. MNRAS, 442:24192433, August 2014.

180. M. Metz, P. Kroupa, and N. I. Libeskind. The Orbital Poles of Milky Way Satellite Galaxies: A Rotationally Supported Disk of Satellites. ApJ, 680:287-294, June 2008.

181. H. Zhao, B. Famaey, F. Lüghausen, and P. Kroupa. Local Group timing in Milgromian dynamics. A past Milky Way-Andromeda encounter at $z>0.8$. A\&A, 557:L3, September 2013.

182. J. Yin, J. L. Hou, N. Prantzos, S. Boissier, R. X. Chang, S. Y. Shen, and B. Zhang. Milky Way versus Andromeda: a tale of two disks. A\&A, 505:497-508, October 2009.

183. F. Hammer, Y. B. Yang, J. L. Wang, M. Puech, H. Flores, and S. Fouquet. Does M31 Result from an Ancient Major Merger? ApJ, 725:542-555, December 2010.

184. M. S. Pawlowski and S. S. McGaugh. Perseus I and the NGC 3109 association in the context of the Local Group dwarf galaxy structures. MNRAS, 440:908-919, May 2014.

185. P. Galianni, F. Patat, J. L. Higdon, S. Mieske, and P. Kroupa. VLT observations of NGC 1097's "dog-leg” tidal stream. Dwarf spheroidals and tidal streams. A\&A, 521:A20, October 2010.

186. I. D. Karachentsev, D. Bautzmann, F. Neyer, R. Polzl, P. Riepe, T. Zilch, and B. Mattern. Three low surface brightness dwarfs discovered around NGC 4631. ArXiv e-prints, January 2014.

187. S. Paudel, P.-A. Duc, P. Côté, J.-C. Cuillandre, L. Ferrarese, E. Ferriere, S. D. J. Gwyn, J. C. Mihos, B. Vollmer, M. L. Balogh, R. G. Carlberg, S. Boissier, A. Boselli, P. R. Durrell, E. Emsellem, L. A. MacArthur, S. Mei, L. Michel-Dansac, and W. van Driel. The Next Generation Virgo Cluster Survey. IV. NGC 4216: A Bombarded Spiral in the Virgo Cluster. ApJ, 767:133, April 2013.

188. K. Chiboucas, B. A. Jacobs, R. B. Tully, and I. D. Karachentsev. Confirmation of Faint Dwarf Galaxies in the M81 Group. AJ, 146:126, November 2013.

189. J. Köppen, C. Weidner, and P. Kroupa. A possible origin of the mass-metallicity relation of galaxies. MNRAS, 375:673-684, February 2007.

190. S. Recchi, F. Calura, and P. Kroupa. The chemical evolution of galaxies within the IGIMF theory: the $[\alpha / \mathrm{Fe}]$ ratios and downsizing. A\&A, 499:711-722, June 2009.

191. E. Spitoni, F. Calura, F. Matteucci, and S. Recchi. The origin of the mass-metallicity relation: an analytical approach. A\&A, 514:A73, May 2010.

192. I. D. Gargiulo, S. A. Cora, N. D. Padilla, A. M. Muñoz Arancibia, A. N. Ruiz, A. A. Orsi, T. E. Tecce, C. Weidner, and G. Bruzual. Chemo-Archeological Downsizing in a Hierarchical Universe: Impact of a Top Heavy IGIMF. ArXiv e-prints, February 2014.

193. S. Recchi, E. Spitoni, F. Matteucci, and G. A. Lanfranchi. The effect of differential galactic winds on the chemical evolution of galaxies. A\&A, 489:555-565, October 2008.

194. M. Boquien, P.-A. Duc, F. Galliano, J. Braine, U. Lisenfeld, V. Charmandaris, and P. N. Appleton. Star Formation in Col- 
lision Debris: Insights from the Modeling of Their Spectral Energy Distribution. AJ, 140:2124-2144, December 2010.

195. E. N. Kirby, J. G. Cohen, P. Guhathakurta, L. Cheng, J. S. Bullock, and A. Gallazzi. The Universal Stellar Mass-Stellar Metallicity Relation for Dwarf Galaxies. ApJ, 779:102, December 2013.

196. S. Recchi and P. Kroupa. The chemical evolution of galaxies with a variable integrated galactic initial mass function. MNRAS, 446:4168-4175, February 2015.

197. A. J. Deason, I. G. McCarthy, A. S. Font, N. W. Evans, C. S. Frenk, V. Belokurov, N. I. Libeskind, R. A. Crain, and T. Theuns. Mismatch and misalignment: dark haloes and satellites of disc galaxies. MNRAS, 415:2607-2625, August 2011.

198. J. Klimentowski, E. L. Łokas, A. Knebe, S. Gottlöber, L. A. Martinez-Vaquero, G. Yepes, and Y. Hoffman. The grouping, merging and survival of subhaloes in the simulated Local Group. MNRAS, 402:1899-1910, March 2010.

199. M. Nichols and J. Bland-Hawthorn. Gas Depletion in Local Group Dwarfs on $250 \mathrm{kpc}$ Scales: Ram Pressure Stripping Assisted by Internal Heating at Early Times. ApJ, 732:17, May 2011.

200. N. I. Libeskind, C. S. Frenk, S. Cole, A. Jenkins, and J. C. Helly. How common is the Milky Way-satellite system alignment? MNRAS, 399:550-558, October 2009.

201. J. Wang, C. S. Frenk, and A. P. Cooper. The spatial distribution of galactic satellites in the $\Lambda$ cold dark matter cosmology. MNRAS, 429:1502-1513, February 2013.

202. H. Bahl and H. Baumgardt. A comparison of the distribution of satellite galaxies around Andromeda and the results of $\Lambda \mathrm{CDM}$ simulations. MNRAS, 438:2916-2923, March 2014.

203. T. Goerdt and A. Burkert. The co-planarity of satellite galaxies delivered by randomly aligned cold mode accretion streams. submitted, ArXiv e-prints, July 2013.

204. N. I. Libeskind, A. Knebe, Y. Hoffman, S. Gottlöber, G. Yepes, and M. Steinmetz. The preferred direction of infalling satellite galaxies in the Local Group. MNRAS, 411:1525-1535, March 2011.

205. E. J. Shaya and R. B. Tully. The formation of Local Group planes of galaxies. MNRAS, 436:2096-2119, December 2013.

206. G. Besla, N. Kallivayalil, L. Hernquist, R. P. van der Marel, T. J. Cox, and D. Kereš. The role of dwarf galaxy interactions in shaping the Magellanic System and implications for Magellanic Irregulars. MNRAS, 421:2109-2138, April 2012.

207. J. Pfeffer and H. Baumgardt. Ultra-compact dwarf galaxy formation by tidal stripping of nucleated dwarf galaxies. MNRAS, 433:1997-2005, August 2013.

208. A. Dekel and Y. Birnboim. Gravitational quenching in massive galaxies and clusters by clumpy accretion. MNRAS, 383:119138, January 2008.

209. A. Dekel, Y. Birnboim, G. Engel, J. Freundlich, T. Goerdt, M. Mumcuoglu, E. Neistein, C. Pichon, R. Teyssier, and E. Zinger. Cold streams in early massive hot haloes as the main mode of galaxy formation. Nature, 457:451-454, January 2009.

210. L. Gao, T. Theuns, and V. Springel. Star forming filaments in warm dark models. ArXiv e-prints, March 2014.

211. S. E. Koposov, J. Yoo, H.-W. Rix, D. H. Weinberg, A. V. Macciò, and J. M. Escudé. A Quantitative Explanation of the Observed Population of Milky Way Satellite Galaxies. ApJ, 696:21792194, May 2009.
212. O. Y. Gnedin and H. Zhao. Maximum feedback and dark matter profiles of dwarf galaxies. MNRAS, 333:299-306, June 2002.

213. F. Lelli, M. Verheijen, and F. Fraternali. Dynamics of Starbursting Dwarf Galaxies. III. A HI study of 18 nearby objects. ArXiv e-prints, April 2014.

214. R. C. Keenan, A. J. Barger, L. L. Cowie, W.-H. Wang, I. Wold, and L. Trouille. Testing for a Large Local Void by Investigating the Near-infrared Galaxy Luminosity Function. ApJ, 754:131, August 2012.

215. D. L. Wiltshire. Average observational quantities in the timescape cosmology. Phys. Rev. D, 80(12):123512, December 2009.

216. M. Milgrom. The Mond Limit from Spacetime Scale Invariance. ApJ, 698:1630-1638, June 2009.

217. P. Kroupa, M. Pawlowski, and M. Milgrom. The Failures of the Standard Model of Cosmology Require a New Paradigm. International Journal of Modern Physics D, 21:30003, December 2012.

218. M. Milgrom. The modified dynamics as a vacuum effect. Physics Letters A, 253:273-279, March 1999.

219. B. L. Ziegler, A. Böhm, K. J. Fricke, K. Jäger, H. Nicklas, R. Bender, N. Drory, A. Gabasch, R. P. Saglia, S. Seitz, J. Heidt, D. Mehlert, C. Möllenhoff, S. Noll, and E. Sutorius. The Evolution of the Tully-Fisher Relation of Spiral Galaxies. ApJL, 564:L69-L72, January 2002.

220. X. Wu and P. Kroupa. The specific frequency and the globular cluster formation efficiency in Milgromian dynamics. MNRAS, 435:1536-1540, October 2013.

221. F. Lüghausen, B. Famaey, and P. Kroupa. A census of the expected properties of classical Milky Way dwarfs in Milgromian dynamics. MNRAS, 441:2497-2507, July 2014.

222. B. Famaey, J.-P. Bruneton, and H. Zhao. Escaping from modified Newtonian dynamics. MNRAS, 377:L79-L82, May 2007.

223. X. Wu and P. Kroupa. The dynamical phase transitions of stellar systems and the corresponding kinematics. MNRAS, 435:728742, October 2013.

224. G. W. Angus, G. Gentile, A. Diaferio, B. Famaey, and K. J. v. d. Heyden. N-body simulations of the Carina dSph in MOND. MNRAS, 440:746-761, March 2014.

225. H. Meyer, E. Lohrmann, S. Schubert, W. Bartel, A. Glazov, B. Löhr, C. Niebuhr, E. Wünsch, L. Jönsson, and G. Kempf. Test of the law of gravitation at small accelerations. General Relativity and Gravitation, 44:2537-2545, October 2012.

226. M. Milgrom. MOND-particularly as modified inertia. ArXiv e-prints, November 2011.

227. M. L. M. Collins, S. C. Chapman, R. M. Rich, M. J. Irwin, J. Peñarrubia, R. A. Ibata, N. Arimoto, A. M. Brooks, A. M. N. Ferguson, G. F. Lewis, A. W. McConnachie, and K. Venn. The scatter about the 'Universal' dwarf spheroidal mass profile: a kinematic study of the M31 satellites And V and And VI. MNRAS, 417:1170-1182, October 2011.

228. X. Hernandez, M. A. Jiménez, and C. Allen. Wide binaries as a critical test of classical gravity. European Physical Journal C, 72:1884, February 2012.

229. R. Scarpa, G. Marconi, G. Carraro, R. Falomo, and S. Villanova. Testing Newtonian gravity with distant globular clusters: NGC 1851 and NGC 1904. A\&A, 525:A148, January 2011.

230. X. Hernandez and M. A. Jiménez. The Outskirts of Globular Clusters as Modified Gravity Probes. ApJ, 750:9, May 2012. 
231. S. Mendoza, X. Hernandez, J. C. Hidalgo, and T. Bernal. A natural approach to extended Newtonian gravity: tests and predictions across astrophysical scales. MNRAS, 411:226-234, February 2011.

232. H. Baumgardt, E. K. Grebel, and P. Kroupa. Using distant globular clusters as a test for gravitational theories. MNRAS, 359:L1L4, May 2005.

233. R. H. Sanders. NGC 2419 does not challenge MOND, Part 2. MNRAS, 422:L21, May 2012.

234. R. H. Sanders and S. S. McGaugh. Modified Newtonian Dynamics as an Alternative to Dark Matter. ARAA, 40:263-317, 2002.

235. J. Bekenstein and M. Milgrom. Does the missing mass problem signal the breakdown of Newtonian gravity? ApJ, 286:7-14, November 1984.

236. M. Milgrom. Quasi-linear formulation of MOND. MNRAS, 403:886-895, April 2010.

237. F. Lüghausen, B. Famaey, and P. Kroupa. Phantom of RAMSES (POR): A new Milgromian dynamics N-body code. Canadian Journal of Physics, 93:232-241, February 2015.

238. O. Tiret and F. Combes. Evolution of spiral galaxies in modified gravity. II. Gas dynamics. A\&A, 483:719-726, June 2008.

239. O. Tiret and F. Combes. Interacting Galaxies with Modified Newtonian Dynamics. In J. G. Funes and E. M. Corsini, editors, Formation and Evolution of Galaxy Disks, volume 396 of Astronomical Society of the Pacific Conference Series, page 259, October 2008.

240. C. Eling, T. Jacobson, and D. Mattingly. Einstein-Aether Theory. ArXiv General Relativity and Quantum Cosmology e-prints, September 2004.

241. G. Gentile, G. W. Angus, B. Famaey, S.-H. Oh, and W. J. G. de Blok. Isolated and non-isolated dwarfs in terms of modified Newtonian dynamics. A\&A, 543:A47, July 2012.

242. F. J. Sánchez-Salcedo, A. M. Hidalgo-Gámez, and E. E. Martínez-García. Slowly Rotating Gas-rich Galaxies in MOdified Newtonian Dynamics (MOND). AJ, 145:61, March 2013.

243. F. J. Sanchez-Salcedo, A. M. Hidalgo-Gamez, and E. E. Martinez-Garcia. The inclination of the dwarf irregular galaxy Holmberg II. ArXiv e-prints, May 2014.

244. E. Corbelli and P. Salucci. Testing modified Newtonian dynamic with Local Group spiral galaxies. MNRAS, 374:1051-1055, January 2007.

245. T. Goerdt, A. Dekel, A. Sternberg, O. Gnat, and D. Ceverino. Detectability of cold streams into high-redshift galaxies by absorption lines. MNRAS, 424:2292-2315, August 2012.

246. D. Rosa-González, E. Terlevich, and R. Terlevich. An empirical calibration of star formation rate estimators. MNRAS, 332:283295, May 2002.

247. D. Calzetti. Star Formation Rate Determinations. In J. H. Knapen, T. J. Mahoney, and A. Vazdekis, editors, Pathways Through an Eclectic Universe, volume 390 of Astronomical Society of the Pacific Conference Series, page 121, June 2008.

248. G. Hensler. How to simulate Galaxy Evolution: strengths and weaknesses of numerical techniques. In H. Wozniak and G. Hensler, editors, EAS Publications Series, volume 44 of EAS Publications Series, pages 3-18, January 2011.

249. J. Silk and G. A. Mamon. The current status of galaxy formation. Research in Astronomy and Astrophysics, 12:917-946, August 2012.
250. S. Recchi. Chemodynamical Simulations of Dwarf Galaxy Evolution. Advances in Astronomy, 2014, 2014.

251. I. Shlosman. Cosmological Evolution of Galaxies, page 555. October 2013.

252. J. Koeppen, C. Theis, and G. Hensler. Self-regulated starformation in chemodynamical models of galaxies. A\&A, 296:99, April 1995.

253. E. Kutdemir, B. L. Ziegler, R. F. Peletier, C. Da Rocha, A. Böhm, and $\mathrm{M}$. Verdugo. Internal kinematics of spiral galaxies in distant clusters. IV. Gas kinematics of spiral galaxies in intermediate redshift clusters and in the field. A\&A, 520:A109, September 2010.

254. D. M. Elmegreen, B. G. Elmegreen, and C. M. Sheets. Chain Galaxies in the Tadpole Advanced Camera for Surveys Field. ApJ, 603:74-81, March 2004.

255. D. M. Elmegreen, B. G. Elmegreen, and A. C. Hirst. Discovery of Face-on Counterparts of Chain Galaxies in the Tadpole Advanced Camera for Surveys Field. ApJL, 604:L21-L23, March 2004.

256. P. Kroupa. Thickening of galactic discs through clustered star formation. MNRAS, 330:707-718, March 2002.

257. B. L. Ziegler and R. Bender. The $\mathrm{Mg}(\mathrm{b})$-sigma relation of elliptical galaxies at $Z$ of about 0.37. MNRAS, 291:527, November 1997.

258. D. Thomas, C. Maraston, R. Bender, and C. Mendes de Oliveira. The Epochs of Early-Type Galaxy Formation as a Function of Environment. ApJ, 621:673-694, March 2005.

259. F. Matteucci. Abundance ratios in ellipticals and galaxy formation. A\&A, 288:57-64, August 1994.

260. A. Vazdekis, R. F. Peletier, J. E. Beckman, and E. Casuso. A New Chemo-evolutionary Population Synthesis Model for Early-Type Galaxies. II. Observations and Results. ApJS, 111:203-232, July 1997.

261. A. Pipino and F. Matteucci. Are dry mergers of ellipticals the way to reconcile model predictions with downsizing? A\&A, 486:763-769, August 2008.

262. P. Kroupa and G. F. Gilmore. Low-mass stars in cooling-flow galaxies. MNRAS, 269:655, August 1994.

263. C. Conroy and P. G. van Dokkum. The Stellar Initial Mass Function in Early-type Galaxies From Absorption Line Spectroscopy. II. Results. ApJ, 760:71, November 2012.

264. C. Spiniello, S. Trager, L. V. E. Koopmans, and C. Conroy. The stellar IMF in early-type galaxies from a non-degenerate set of optical line indices. MNRAS, 438:1483-1499, February 2014.

265. P. Kroupa, C. Weidner, J. Pflamm-Altenburg, I. Thies, J. Dabringhausen, M. Marks, and T. Maschberger. The stellar and sub-stellar initial mass function of simple and composite popu page 115. 2013.

266. C. Weidner, I. Ferreras, A. Vazdekis, and F. La Barbera. The (galaxy-wide) IMF in giant elliptical galaxies: from top to bottom. MNRAS, 435:2274-2280, November 2013.

267. R. J. Smith and J. R. Lucey. A giant elliptical galaxy with a lightweight initial mass function. MNRAS, 434:1964-1977, September 2013.

268. M. B. Peacock, S. E. Zepf, T. J. Maccarone, A. Kundu, A. H. Gonzalez, B. D. Lehmer, and C. Maraston. Evidence for a Constant Initial Mass Function in Early-type Galaxies Based on Their X-Ray Binary Populations. ApJ, 784:162, April 2014. 
269. T. J. Maccarone. Destruction of wide binary stars in low-mass elliptical galaxies: implications for initial mass function estimates. MNRAS, April 2014.

270. M. Cappellari, R. M. McDermid, K. Alatalo, L. Blitz, M. Bois, F. Bournaud, M. Bureau, A. F. Crocker, R. L. Davies, T. A. Davis, P. T. de Zeeuw, P.-A. Duc, E. Emsellem, S. Khochfar, D. Krajnović, H. Kuntschner, P.-Y. Lablanche, R. Morganti, T. Naab, T. Oosterloo, M. Sarzi, N. Scott, P. Serra, A.-M. Weijmans, and L. M. Young. Systematic variation of the stellar initial mass function in early-type galaxies. Nature, 484:485-488, April 2012.

271. P. Kroupa. Inverse dynamical population synthesis and star formation. MNRAS, 277:1491, December 1995.

272. C. J. Lada and E. A. Lada. Embedded Clusters in Molecular Clouds. ARAA, 41:57-115, 2003.

273. W. Brandner. The Starburst Cluster Westerlund 1 and its Galactic Siblings - Observation Confronts Theory. In H. Beuther, H. Linz, and T. Henning, editors, Massive Star Formation: Observations Confront Theory, volume 387 of Astronomical Society of the Pacific Conference Series, page 369, May 2008.

274. P. Kroupa and J. Bouvier. The dynamical evolution of TaurusAuriga-type aggregates. MNRAS, 346:343-353, December 2003.

275. P. Kroupa, S. Aarseth, and J. Hurley. The formation of a bound star cluster: from the Orion nebula cluster to the Pleiades. MNRAS, 321:699-712, March 2001.

276. S. Banerjee and P. Kroupa. Did the Infant R136 and NGC 3603 Clusters Undergo Residual Gas Expulsion? ApJ, 764:29, February 2013.

277. S. Banerjee and P. Kroupa. A perfect starburst cluster made in one go: the NGC 3603 young cluster. ArXiv e-prints, March 2014.

278. J. Malinen, M. Juvela, M. G. Rawlings, D. Ward-Thompson, P. Palmeirim, and P. André. Profiling filaments: comparing nearinfrared extinction and submillimetre data in TMC-1. A\&A, 544:A50, August 2012.

279. M. Marks and P. Kroupa. Inverse dynamical population synthesis. Constraining the initial conditions of young stellar clusters by studying their binary populations. A\&A, 543:A8, July 2012.

280. M. Marks, P. Kroupa, J. Dabringhausen, and M. S. Pawlowski. Evidence for top-heavy stellar initial mass functions with increasing density and decreasing metallicity. MNRAS, 422:2246-2254, May 2012.

281. C. Weidner, P. Kroupa, J. Pflamm-Altenburg, and A. Vazdekis. The galaxy-wide initial mass function of dwarf late-type to massive early-type galaxies. MNRAS, 436:3309-3320, December 2013.

282. P. Kroupa. The Fundamental Building Blocks of Galaxies. In C. Turon, K. S. O'Flaherty, and M. A. C. Perryman, editors, The Three-Dimensional Universe with Gaia, volume 576 of ESA Special Publication, page 629, January 2005.

283. H. D. Tran, M. Sirianni, H. C. Ford, G. D. Illingworth, M. Clampin, G. Hartig, R. H. Becker, R. L. White, F. Bartko, N. Benítez, J. P. Blakeslee, R. Bouwens, T. J. Broadhurst, R. Brown, C. Burrows, E. Cheng, N. Cross, P. D. Feldman, M. Franx, D. A. Golimowski, C. Gronwall, L. Infante, R. A. Kimble, J. Krist, M. Lesser, D. Magee, A. R. Martel, W. J. McCann, G. R. Meurer, G. Miley, M. Postman, P. Rosati, W. B. Sparks, and Z. Tsvetanov. Advanced Camera for Surveys Ob- servations of Young Star Clusters in the Interacting Galaxy UGC 10214. ApJ, 585:750-755, March 2003.

284. R. C. Brüns, P. Kroupa, M. Fellhauer, M. Metz, and P. Assmann. A parametric study on the formation of extended star clusters and ultra-compact dwarf galaxies. A\&A, 529:A138, May 2011.

285. A. W. McConnachie. The Observed Properties of Dwarf Galaxies in and around the Local Group. AJ, 144:4, July 2012.

286. M. Marks and P. Kroupa. Dynamical population synthesis: constructing the stellar single and binary contents of galactic field populations. MNRAS, 417:1702-1714, November 2011.

287. N. Bastian, K. R. Covey, and M. R. Meyer. A Universal Stellar Initial Mass Function? A Critical Look at Variations. ARAA, 48:339-389, September 2010.

288. C. Weidner, P. Kroupa, and J. Pflamm-Altenburg. The $\mathrm{m}_{\max }{ }^{-}$ $\mathrm{M}_{e c l}$ relation, the IMF and IGIMF: probabilistically sampled functions. MNRAS, 434:84-101, September 2013.

289. C. Weidner, P. Kroupa, and S. S. Larsen. Implications for the formation of star clusters from extragalactic star formation rates. MNRAS, 350:1503-1510, June 2004.

290. J. Dabringhausen, P. Kroupa, and H. Baumgardt. A top-heavy stellar initial mass function in starbursts as an explanation for the high mass-to-light ratios of ultra-compact dwarf galaxies. MNRAS, 394:1529-1543, April 2009.

291. J. Dabringhausen, M. Fellhauer, and P. Kroupa. Mass loss and expansion of ultra compact dwarf galaxies through gas expulsion and stellar evolution for top-heavy stellar initial mass functions. MNRAS, 403:1054-1071, April 2010.

292. J. Dabringhausen, P. Kroupa, J. Pflamm-Altenburg, and S. Mieske. Low-mass X-Ray Binaries Indicate a Top-heavy Stellar Initial Mass Function in Ultracompact Dwarf Galaxies. ApJ, 747:72, March 2012.

293. N. Prantzos and C. Charbonnel. On the self-enrichment scenario of galactic globular clusters: constraints on the IMF. A\&A, 458:135-149, October 2006.

294. B. K. Gibson and F. Matteucci. Infall models of elliptical galaxies: further evidence for a top-heavy initial mass function. MNRAS, 291:L8-L12, October 1997.

295. S. K. Ballero, P. Kroupa, and F. Matteucci. Testing the universal stellar IMF on the metallicity distribution in the bulges of the Milky Way and M 31. A\&A, 467:117-121, May 2007.

296. S. K. Ballero, F. Matteucci, L. Origlia, and R. M. Rich. Formation and evolution of the Galactic bulge: constraints from stellar abundances. A\&A, 467:123-136, May 2007.

297. E. A. Hoversten and K. Glazebrook. Evidence for a Nonuniversal Stellar Initial Mass Function from the Integrated Properties of SDSS Galaxies. ApJ, 675:163-187, March 2008.

298. G. R. Meurer, O. I. Wong, J. H. Kim, D. J. Hanish, T. M. Heckman, J. Werk, J. Bland-Hawthorn, M. A. Dopita, M. A. Zwaan, B. Koribalski, M. Seibert, D. A. Thilker, H. C. Ferguson, R. L. Webster, M. E. Putman, P. M. Knezek, M. T. Doyle, M. J. Drinkwater, C. G. Hoopes, V. A. Kilborn, M. Meyer, E. V. Ryan-Weber, R. C. Smith, and L. Staveley-Smith. Evidence for a Nonuniform Initial Mass Function in the Local Universe. ApJ, 695:765-780, April 2009.

299. M. L. P. Gunawardhana, A. M. Hopkins, R. G. Sharp, S. Brough, E. Taylor, J. Bland-Hawthorn, C. Maraston, R. J. Tuffs, C. C. Popescu, D. Wijesinghe, D. H. Jones, S. Croom, E. Sadler, S. Wilkins, S. P. Driver, J. Liske, P. Norberg, I. K. Baldry, S. P. Bamford, J. Loveday, J. A. Peacock, A. S. G. Robotham, D. B. Zucker, Q. A. Parker, C. J. Conselice, E. Cameron, C. S. Frenk, 
D. T. Hill, L. S. Kelvin, K. Kuijken, B. F. Madore, B. Nichol, H. R. Parkinson, K. A. Pimbblet, M. Prescott, W. J. Sutherland, D. Thomas, and E. van Kampen. Galaxy and Mass Assembly (GAMA): the star formation rate dependence of the stellar initial mass function. MNRAS, 415:1647-1662, August 2011.

300. P. P. Papadopoulos, W.-F. Thi, F. Miniati, and S. Viti. Extreme cosmic ray dominated regions: a new paradigm for high star formation density events in the Universe. MNRAS, 414:17051714, June 2011.

301. P. P. Papadopoulos and W.-F. Thi. The Initial Conditions of Star Formation: Cosmic Rays as the Fundamental Regulators. In D. F. Torres and O. Reimer, editors, Cosmic Rays in Star-Forming Environments, volume 34 of Advances in Solid State Physics, page 41, 2013.

302. P. Kroupa and C. Weidner. Galactic-Field Initial Mass Functions of Massive Stars. ApJ, 598:1076-1078, December 2003.

303. C. Weidner and P. Kroupa. The Variation of Integrated Star Initial Mass Functions among Galaxies. ApJ, 625:754-762, June 2005.

304. F. Calura, S. Recchi, F. Matteucci, and P. Kroupa. Effects of the integrated galactic IMF on the chemical evolution of the solar neighbourhood. MNRAS, 406:1985-1999, August 2010.

305. S. Recchi, F. Calura, B. K. Gibson, and P. Kroupa. The $[\alpha / \mathrm{Fe}]$ ratios of very metal-poor stars within the integrated galactic initial mass function theory. MNRAS, 437:994-1008, January 2014.

306. K. Bekki. Simulating galaxy evolution with a non-universal stellar initial mass function. MNRAS, 436:2254-2275, December 2013.

307. J. C. Lee, A. Gil de Paz, C. Tremonti, R. C. Kennicutt, Jr., S. Salim, M. Bothwell, D. Calzetti, J. Dalcanton, D. Dale, C. Engelbracht, S. J. J. G. Funes, B. Johnson, S. Sakai, E. Skillman, L. van Zee, F. Walter, and D. Weisz. Comparison of $\mathrm{H} \alpha$ and UV Star Formation Rates in the Local Volume: Systematic Discrepancies for Dwarf Galaxies. ApJ, 706:599-613, November 2009.

308. C. Weidner, P. Kroupa, and J. Pflamm-Altenburg. Sampling methods for stellar masses and the mmax-Mecl relation in the starburst dwarf galaxy NGC 4214. ArXiv e-prints, April 2014.

309. A. W. McConnachie, N. Arimoto, M. Irwin, and E. Tolstoy. The stellar content of the isolated transition dwarf galaxy DDO210. MNRAS, 373:715-728, December 2006.

310. W.-H. Hsu, L. Hartmann, L. Allen, J. Hernández, S. T. Megeath, G. Mosby, J. J. Tobin, and C. Espaillat. The Low-mass Stellar Population in L1641: Evidence for Environmental Dependence of the Stellar Initial Mass Function. ApJ, 752:59, June 2012.

311. H. Kirk, S. S. R. Offner, and K. J. Redmond. The formation and evolution of small star clusters. MNRAS, 439:1765-1780, February 2014.

312. P. Kroupa. The Initial Mass Function of Stars: Evidence for Uniformity in Variable Systems. Science, 295:82-91, January 2002.

313. J. Pflamm-Altenburg, R. A. González-Lópezlira, and P. Kroupa. The galactocentric radius dependent upper mass limit of young star clusters: stochastic star formation ruled out. MNRAS, 435:2604-2609, November 2013.

314. P. Vaisanen, Z. Randriamanakoto, A. Escala, E. Kankare, A. Kniazev, J. K. Kotilainen, S. Mattila, R. Ramphul, S. Ryder, and A. Tekola. Super Star Clusters in Luminous Infrared Galaxies: the SUNBIRD Survey. ArXiv e-prints, February 2014.
315. J. Pflamm-Altenburg, C. Weidner, and P. Kroupa. Diverging UV and $\mathrm{H} \alpha$ fluxes of star-forming galaxies predicted by the IGIMF theory. MNRAS, 395:394-400, May 2009.

316. J. Pflamm-Altenburg and P. Kroupa. The Fundamental Gas Depletion and Stellar-Mass Buildup Times of Star-Forming Galaxies. ApJ, 706:516-524, November 2009.

317. K. Guo, X. Z. Zheng, and H. Fu. The Intrinsic Scatter along the Main Sequence of Star-forming Galaxies at $\mathrm{z}^{\sim}$ 0.7. ApJ, 778:23, November 2013.

318. S. S. McGaugh and W. J. G. de Blok. Gas Mass Fractions and the Evolution of Spiral Galaxies. ApJ, 481:689, May 1997.

319. S. Ploeckinger, S. Recchi, G. Hensler, and P. Kroupa. Chemodynamical evolution of tidal dwarf galaxies. II. The long-term evolution and influence of a tidal field. submitted, 2015.

320. G. W. Angus. Is an $11 \mathrm{eV}$ sterile neutrino consistent with clusters, the cosmic microwave background and modified Newtonian dynamics? MNRAS, 394:527-532, March 2009.

321. G. W. Angus and A. Diaferio. The abundance of galaxy clusters in modified Newtonian dynamics: cosmological simulations with massive neutrinos. MNRAS, 417:941-949, October 2011.

322. G. W. Angus, A. Diaferio, B. Famaey, and K. J. van der Heyden. Cosmological simulations in MOND: the cluster scale halo mass function with light sterile neutrinos. MNRAS, 436:202-211, November 2013.

323. C. Llinares, A. Knebe, and H. Zhao. Cosmological structure formation under MOND: a new numerical solver for Poisson's equation. MNRAS, 391:1778-1790, December 2008.

324. S. Dodelson. The Real Problem with MOND. International Journal of Modern Physics D, 20:2749-2753, 2011.

325. G. W. Angus, B. Famaey, and A. Diaferio. Equilibrium configurations of $11 \mathrm{eV}$ sterile neutrinos in MONDian galaxy clusters. MNRAS, 402:395-408, February 2010.

326. H.-J. Fahr and J. H. Zoennchen. The "writing on the cosmic wall": Is there a straightforward explanation of the cosmic microwave background? Annalen der Physik, 18:699-721, 2009.

327. V. Belokurov, D. B. Zucker, N. W. Evans, J. T. Kleyna, S. Koposov, S. T. Hodgkin, M. J. Irwin, G. Gilmore, M. I. Wilkinson, M. Fellhauer, D. M. Bramich, P. C. Hewett, S. Vidrih, J. T. A. De Jong, J. A. Smith, H.-W. Rix, E. F. Bell, R. F. G. Wyse, H. J. Newberg, P. A. Mayeur, B. Yanny, C. M. Rockosi, O. Y. Gnedin, D. P. Schneider, T. C. Beers, J. C. Barentine, H. Brewington, J. Brinkmann, M. Harvanek, S. J. Kleinman, J. Krzesinski, D. Long, A. Nitta, and S. A. Snedden. Cats and Dogs, Hair and a Hero: A Quintet of New Milky Way Companions. ApJ, 654:897-906, January 2007.

328. C. J. Grillmair. Four New Stellar Debris Streams in the Galactic Halo. ApJ, 693:1118-1127, March 2009.

329. J. L. Carlin, C. J. Grillmair, R. R. Muñoz, D. L. Nidever, and S. R. Majewski. Kinematics and Metallicities in the Boötes III Stellar Overdensity: A Disrupted Dwarf Galaxy? ApJL, 702:L9-L13, September 2009.

330. S. S. McGaugh and J. Wolf. Local Group Dwarf Spheroidals: Correlated Deviations from the Baryonic Tully-Fisher Relation. ApJ, 722:248-261, October 2010.

331. G. W. Angus, G. Gentile, A. Diaferio, B. Famaey, and K. J. v. d. Heyden. N-body simulations of the Carina dSph in MOND. MNRAS, 440:746-761, May 2014.

332. E. Pompei and A. Iovino. Galaxy evolution in a new Southern Compact Groups sample. In V. Avila-Reese, C. Firmani, C. S. Frenk, and C. Allen, editors, Revista Mexicana de Astronomia y 
Astrofisica Conference Series, volume 17 of Revista Mexicana de Astronomia y Astrofisica, vol. 27, pages 35-35, June 2003.

333. E. Pompei and A. Iovino. Compact Groups of Galaxies: Small, Dense, and Elusive. In L. Verdes-Montenegro, A. Del Olmo, and J. Sulentic, editors, Galaxies in Isolation: Exploring Nature Versus Nurture, volume 421 of Astronomical Society of the Pacific Conference Series, page 279, October 2010.

334. M. Spavone, E. Iodice, M. Arnaboldi, G. Longo, and O. Gerhard. Chemical abundances of the PRGs UGC 7576 and UGC 9796. I. Testing the formation scenario. A\&A, 531:A21, July 2011.

335. S. Kazantzidis, L. Mayer, C. Mastropietro, J. Diemand, J. Stadel, and B. Moore. Density Profiles of Cold Dark Matter Substructure: Implications for the Missing-Satellites Problem. ApJ, 608:663-679, June 2004

336. S. McGaugh and M. Milgrom. Andromeda Dwarfs in Light of MOND. II. Testing Prior Predictions. ApJ, 775:139, October 2013.

337. F. Lüghausen, B. Famaey, P. Kroupa, G. Angus, F. Combes, G. Gentile, O. Tiret, and H. Zhao. Polar ring galaxies as tests of gravity. MNRAS, 432:2846-2853, July 2013.

338. L. Blanchet and A. Le Tiec. Dipolar dark matter and dark energy. Phys. Rev. D, 80(2):023524, July 2009.

339. L. Blanchet and L. Bernard. Phenomenology of MOND and gravitational polarization. International Journal of Modern Physics Conference Series, 30:60271, May 2014.

340. A. J. K. Chua, D. T. Wickramasinghe, and L. Ferrario. Galactic escape speeds in mirror and cold dark matter models. European Physical Journal C, 73:2259, January 2013.

341. J. W. Moffat. Scalar tensor vector gravity theory. Journal of Cosmology and Astroparticle Physics, 3:4, March 2006.

342. S. Trippe. A Derivation of Modified Newtonian Dynamics. Journal of Korean Astronomical Society, 46:93-96, April 2013.

343. S. Trippe. Can Massive Gravity Explain the Mass DiscrepancyAcceleration Relation of Disk Galaxies? Journal of Korean Astronomical Society, 46:133-140, June 2013.

344. F. W. Hehl and B. Mashhoon. Formal framework for a nonlocal generalization of Einstein's theory of gravitation. Phys. Rev. D, 79(6):064028, March 2009.

345. F. W. Hehl and B. Mashhoon. Nonlocal gravity simulates dark matter. Physics Letters B, 673:279-282, March 2009.

346. S. Rahvar and B. Mashhoon. Observational Tests of Nonlocal Gravity: Galaxy Rotation Curves and Clusters of Galaxies. ArXiv e-prints, January 2014.

\section{A. Acronyms}

This appendix lists the used acronyms, their meaning and first-time occurrence in the text.

\begin{tabular}{|c|c|c|}
\hline Acronym & Meaning & Sec. \\
\hline$\overline{\mathrm{BH}}$ & black hole & 2 \\
\hline BTFR & baryonic TullyFisher relation & 4.1.1 \\
\hline CDM & cold dark matter & 1 \\
\hline $\mathrm{CMB}$ & cosmic microwave background & 2 \\
\hline DDG & dual dwarf galaxy theorem & 3.3 \\
\hline $\mathrm{dE}$ & dwarf elliptical galaxy & 3.2 \\
\hline DoS & $\begin{array}{l}\text { disk of satellites of the Galaxy; it contains } \\
\text { all known satellite galaxies of the Galaxy }\end{array}$ & 4.2 .2 \\
\hline $\mathrm{DM}$ & $\begin{array}{l}\text { exotic (cold or warm) dark matter particles; } \\
\text { not contained in the SMoPP }\end{array}$ & 1 \\
\hline $\mathrm{dSph}$ & $\begin{array}{l}\text { dwarf spheroidal galaxy; baryonic mass } \\
10^{5} \lesssim M / M_{\odot} \lesssim 10^{7} ; \text { known as common } \\
\text { satellites around nearby larger galaxies; } \\
\text { MW dSph satellites were discovered with } \\
\text { photographic plates and are the "classical" } \\
\text { satellite galaxies }\end{array}$ & 3.1 \\
\hline $\mathrm{E}$ & elliptical & 2 \\
\hline $\mathrm{EC}$ & embedded cluster & 7.2 \\
\hline ECMF & embedded cluster mass function & 7.2 \\
\hline EFE & external field effect & 6.1 \\
\hline GC & globular star cluster & 5.1.1.1 \\
\hline GPoA & $\begin{array}{l}\text { great plane of Andromeda, sometimes also } \\
\text { called the VTDS }\end{array}$ & 5.1.1.2 \\
\hline GR & Einstein's theory of general relativity & 2 \\
\hline IGIMF & $\begin{array}{l}\text { integrated galactic IMF; the IMF of a whole } \\
\text { galaxy }\end{array}$ & 5.1 .2 \\
\hline IMF & initial mass function & 5.1 .2 \\
\hline ISM & inter stellar medium & 5.2 \\
\hline$\Lambda \mathrm{CDM}$ & $\begin{array}{l}\text { Lambda }(\Lambda) \text { cold dark matter cosmological } \\
\text { model; the SMoC }\end{array}$ & 2 \\
\hline LMC & Large Magellanic Cloud & 5.1 .3 \\
\hline LMXB & low-mass X-ray binary & 7.1 \\
\hline$\Lambda \mathrm{WDM}$ & $\begin{array}{l}\text { Lambda }(\Lambda) \text { warm dark matter cosmolog- } \\
\text { ical model; here also referred to as the } \\
\text { SMoC as it is virtually identical to the } \\
\text { LCDM model apart from the exotic DM } \\
\text { particles having a longer streaming length } \\
\text { initially }\end{array}$ & 2 \\
\hline MDA & mass-discrepancy-acceleration & 6 \\
\hline MOND & $\begin{array}{l}\text { modified Newtonian dynamics; Milgrom's } \\
\text { effective theory of classical gravitational } \\
\text { dynamics }\end{array}$ & 6.3 \\
\hline MW & Milky Way, the Galaxy & 3.1 \\
\hline M31 & Andromeda galaxy & 5.1 .2 \\
\hline M33 & Triangulum & 6.4 \\
\hline PDF & probability density distribution function & 7.2 \\
\hline PDG & $\begin{array}{l}\text { primordial dwarf galaxy; dominated by DM } \\
\text { if the } \mathrm{SMoC} \text { is true }\end{array}$ & 3.1 \\
\hline POR & Phantom of Ramses simulation code [237] & 6.3 \\
\hline $\mathrm{R}(\mathrm{M}) \mathrm{R}$ & radius-mass relation & 4.1 .2 \\
\hline SFR & star formation rate & 4.2 .4 \\
\hline Sgr & Sagittarius & 4.2 .1 \\
\hline SID & scale-invariant dynamics & 6 \\
\hline SMC & Small Magellanic Cloud & 5.1 .3 \\
\hline SMoC & $\begin{array}{l}\text { The standard model of cosmology; LCDM } \\
\text { or LWDM models }\end{array}$ & 1 \\
\hline SMoPP & the standard model of particle physics & 1 \\
\hline TDG & $\begin{array}{l}\text { tidal dwarf galaxy; cannot have a dynami- } \\
\text { cally significant DM content if the SMoC is } \\
\text { true }\end{array}$ & 3.2 \\
\hline
\end{tabular}


Acronym Meaning

\begin{tabular}{lll} 
Acronym & Meaning & Sec. \\
\hline UCD & ultra compact dwarf galaxy & 5.1 .3 \\
UFD & ultra faint dwarf galaxy; baryonic mass & 3.1 \\
& $10^{3} \lesssim M / M_{\odot} \lesssim 10^{5} ;$ MW UFDs are dis- \\
& $\begin{array}{l}\text { covered with deep automatic/robotic sky } \\
\text { surveys }\end{array}$ \\
& $\begin{array}{l}\text { vast polar structure of the MW; it contains } \\
\text { all known satellite galaxies, young halo }\end{array}$ & \\
& $\begin{array}{l}\text { globular clusters and a large fraction of the } \\
\text { known stellar and gas streams }\end{array}$ & \\
& warm dark matter & 1 \\
\hline
\end{tabular}

